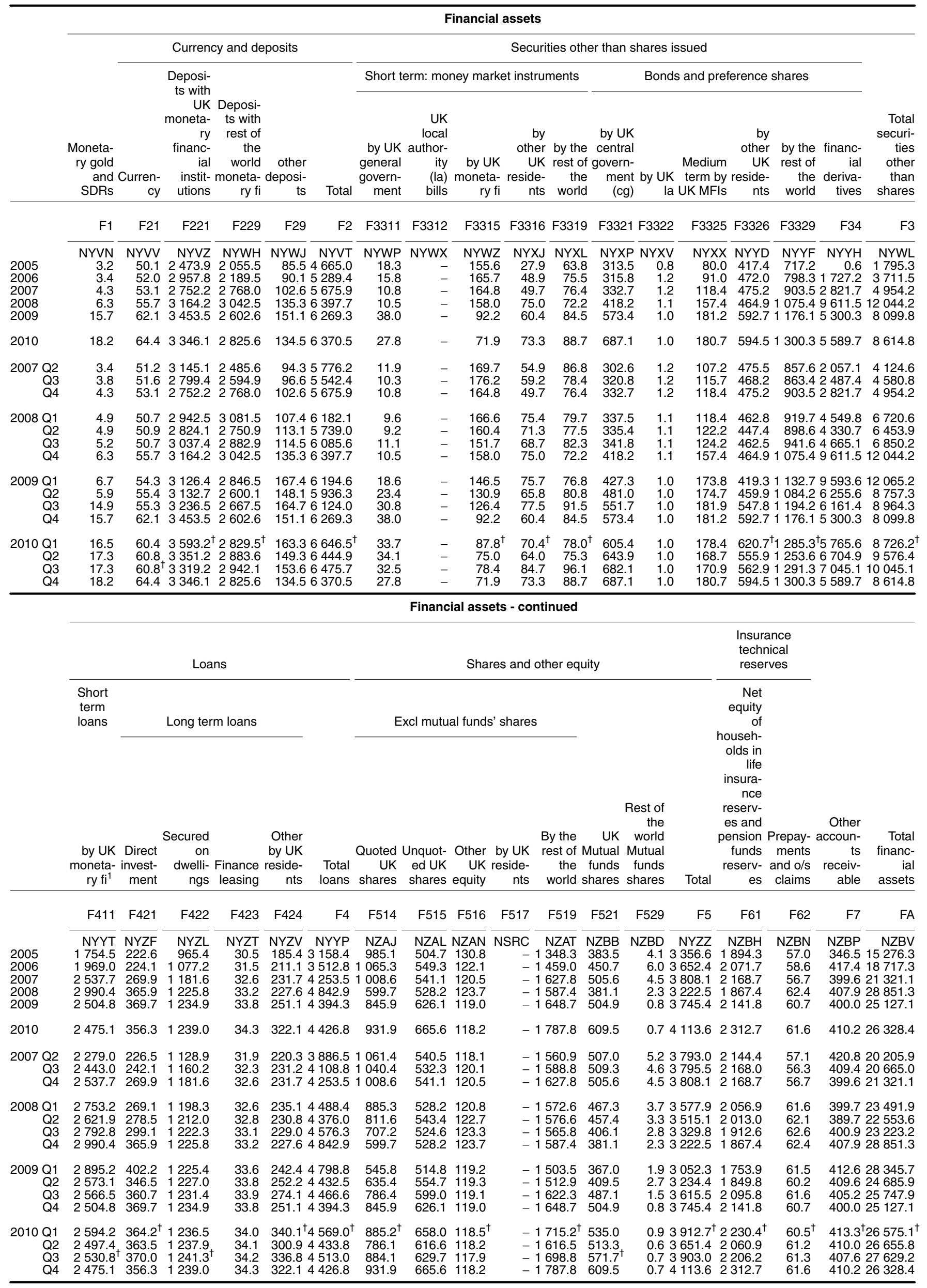

1 Excluding loans secured on dwellings and finance leasing. 


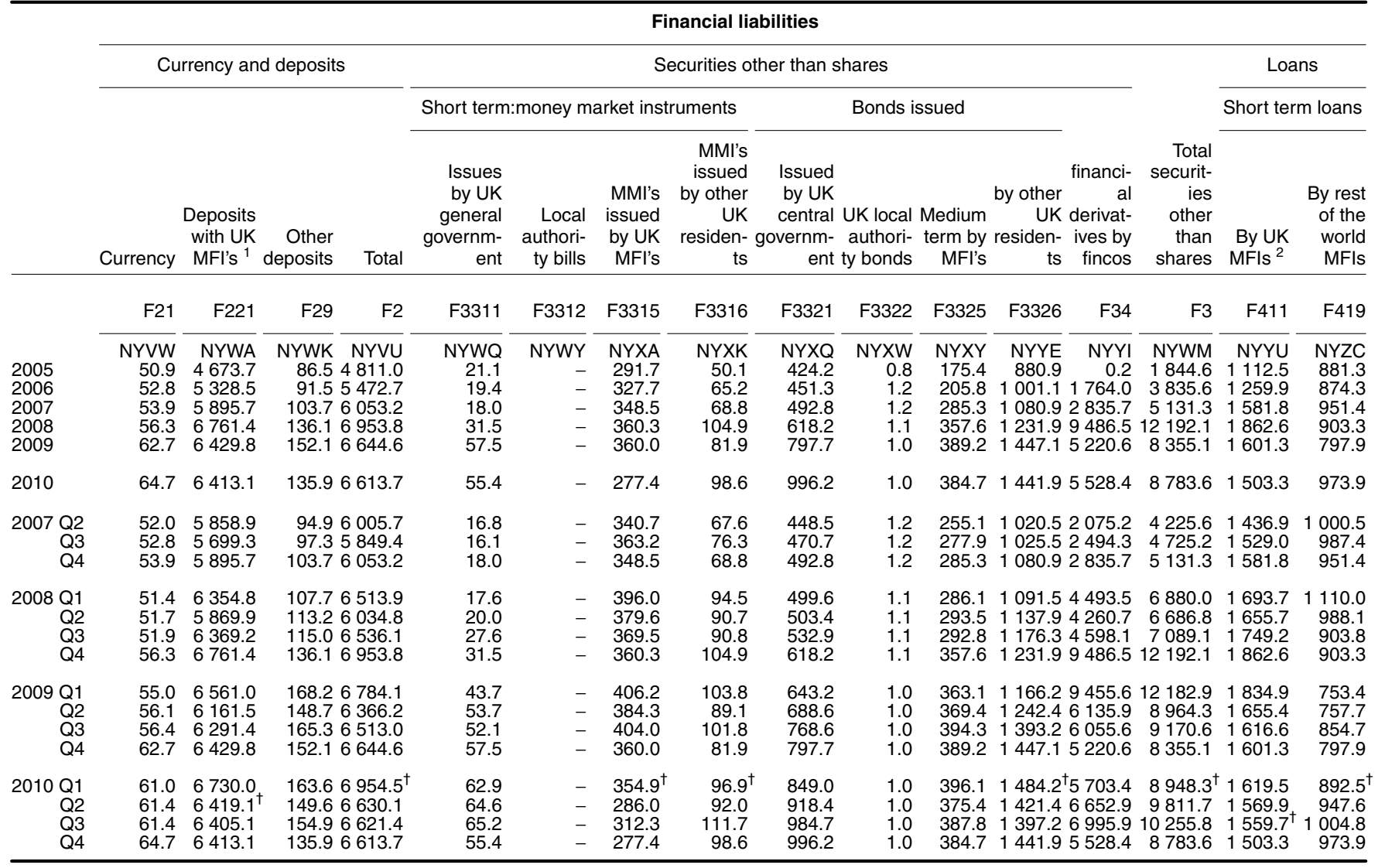

Financial liabilities - continued

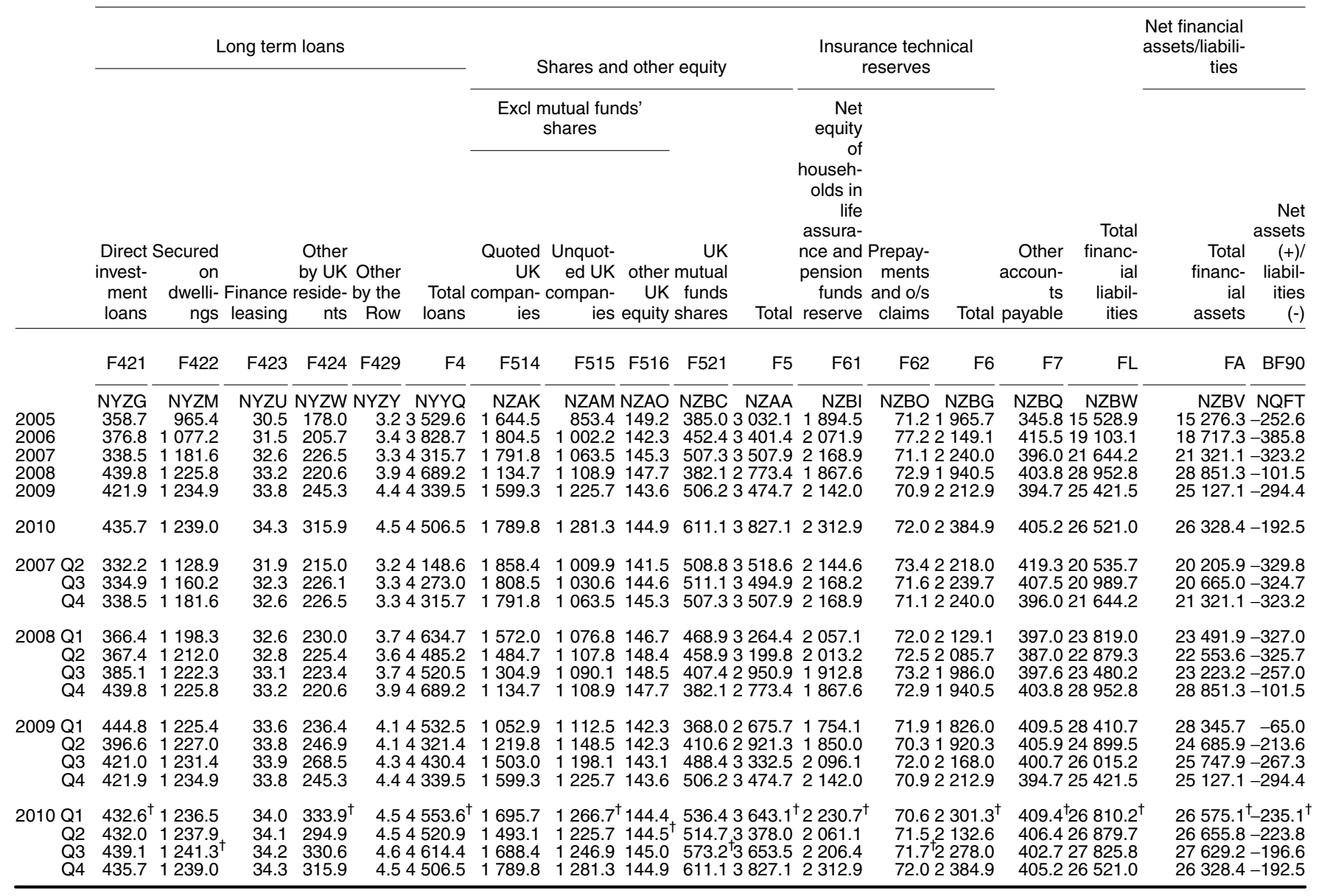




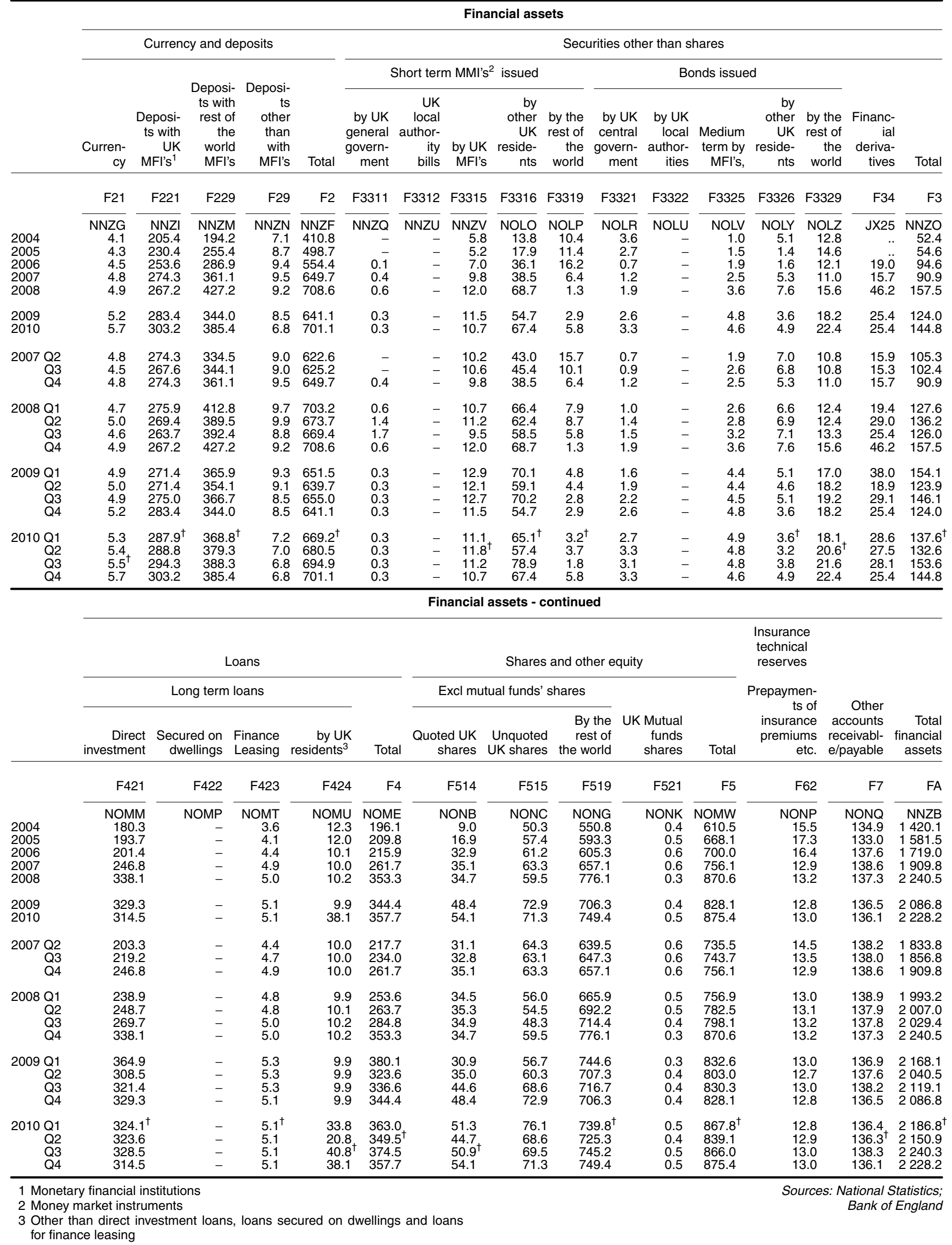




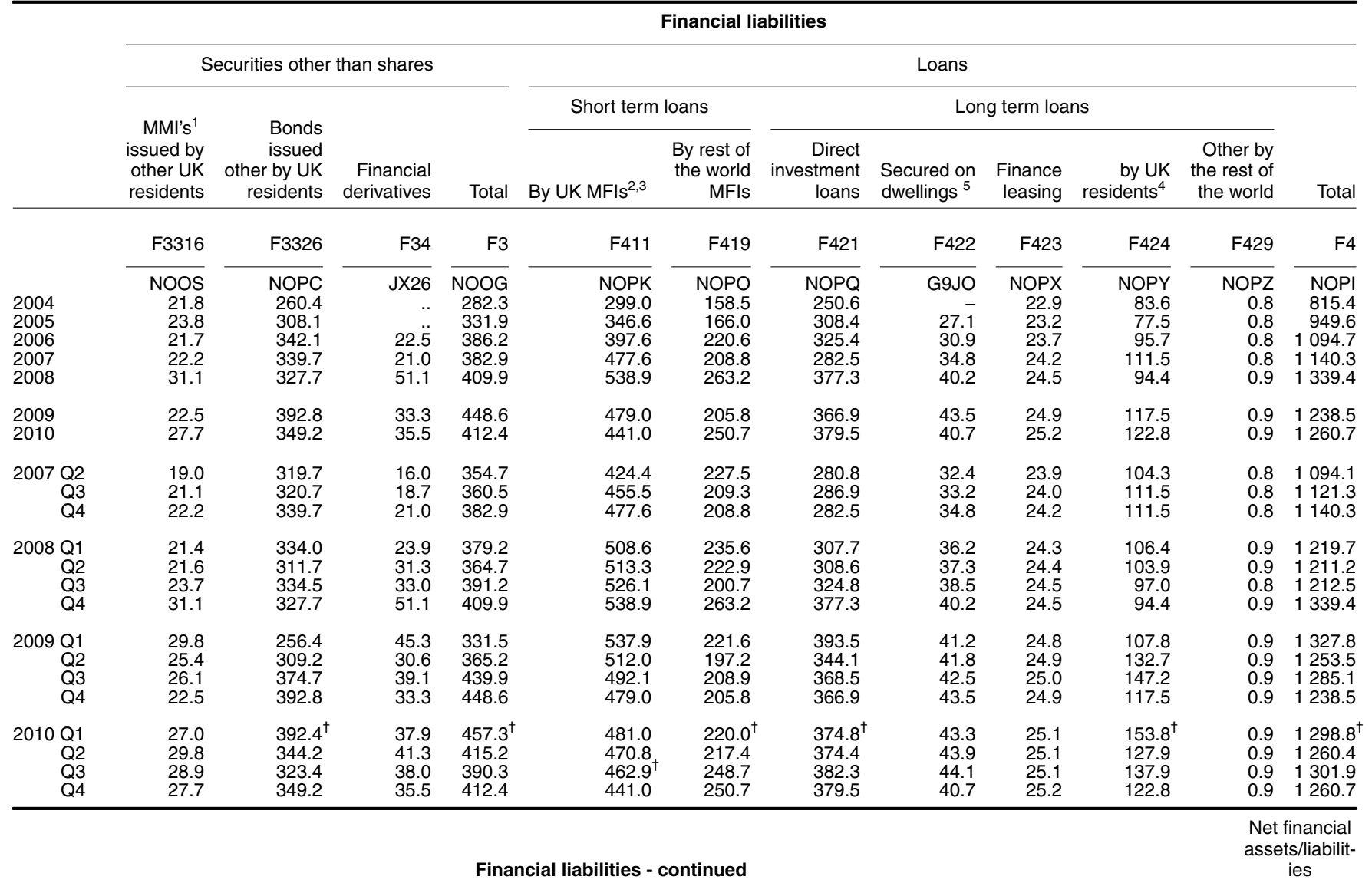

Financial liabilities - continued

\begin{tabular}{|c|c|c|c|c|c|c|c|c|}
\hline & \multirow{2}{*}{\multicolumn{4}{|c|}{ Shares and other equity }} & & \multirow[b]{4}{*}{$\begin{array}{r}\text { Total financial } \\
\text { liabilities }\end{array}$} & \multirow[b]{4}{*}{$\begin{array}{r}\text { Total financial } \\
\text { assets }\end{array}$} & \multirow{4}{*}{$\begin{array}{r}\text { Net assets } \\
(+) / \text { liabilities- } \\
(-)\end{array}$} \\
\hline & & & & & \multirow{3}{*}{$\begin{array}{r}\text { Other accounts } \\
\text { receivable/paya- } \\
\text { ble }\end{array}$} & & & \\
\hline & \multicolumn{3}{|c|}{ Excl mutual funds' shares } & \multirow[b]{2}{*}{ Total } & & & & \\
\hline & $\begin{array}{l}\text { Quoted UK } \\
\text { companies }\end{array}$ & $\begin{array}{r}\text { Unquoted UK } \\
\text { companies }\end{array}$ & other UK equity & & & & & \\
\hline & F514 & F515 & F516 & F5 & $\mathrm{F} 7$ & $\mathrm{FL}$ & FA & BF90 \\
\hline $\begin{array}{l}2004 \\
2005 \\
2006 \\
2007 \\
2008\end{array}$ & $\begin{array}{r}\text { NOQF } \\
1080.2 \\
1235.4 \\
1318.7 \\
1366.1 \\
917.9\end{array}$ & $\begin{array}{r}\text { NOQG } \\
423.9 \\
515.0 \\
611.1 \\
672.6 \\
619.2\end{array}$ & $\begin{array}{r}\text { NOQH } \\
132.4 \\
149.2 \\
142.3 \\
145.3 \\
147.7\end{array}$ & $\begin{array}{r}\text { NOQA } \\
1636.6 \\
1699.6 \\
2072.1 \\
2184.0 \\
1684.8\end{array}$ & $\begin{array}{r}\text { NOQU } \\
154.7 \\
159.6 \\
162.4 \\
163.0 \\
162.4\end{array}$ & $\begin{array}{r}\text { NONT } \\
2888.9 \\
3 \quad 340.7 \\
3 \quad 715.4 \\
3 \quad 870.3 \\
3596.5\end{array}$ & $\begin{array}{r}\text { NNZB } \\
1420.1 \\
1581.5 \\
1719.0 \\
1909.8 \\
2240.5\end{array}$ & $\begin{array}{r}\text { NYOM } \\
-1468.8 \\
-1759.2 \\
-1996.4 \\
-1960.5 \\
-1356.0\end{array}$ \\
\hline $\begin{array}{l}2009 \\
2010\end{array}$ & $\begin{array}{l}1212.6 \\
1373.7\end{array}$ & $\begin{array}{l}633.5 \\
675.3\end{array}$ & $\begin{array}{l}143.6 \\
144.9\end{array}$ & $\begin{array}{l}1989.7 \\
2193.9\end{array}$ & $\begin{array}{l}162.3 \\
164.8\end{array}$ & $\begin{array}{l}3839.1 \\
4031.7\end{array}$ & $\begin{array}{l}2086.8 \\
2228.2\end{array}$ & $\begin{array}{l}-1752.3 \\
-1803.5\end{array}$ \\
\hline $\begin{array}{r}2007 \text { Q2 } \\
\text { Q3 } \\
\text { Q4 }\end{array}$ & $\begin{array}{l}1386.3 \\
1361.9 \\
1366.1\end{array}$ & $\begin{array}{l}642.9 \\
662.1 \\
672.6\end{array}$ & $\begin{array}{l}141.5 \\
144.6 \\
145.3\end{array}$ & $\begin{array}{l}2170.7 \\
2168.6 \\
2184.0\end{array}$ & $\begin{array}{l}163.8 \\
162.1 \\
163.0\end{array}$ & $\begin{array}{l}3783.2 \\
3812.5 \\
3870.3\end{array}$ & $\begin{array}{l}1833.8 \\
1856.8 \\
1909.8\end{array}$ & $\begin{array}{l}-1949.4 \\
-1955.6 \\
-1960.5\end{array}$ \\
\hline $\begin{array}{r}2008 \text { Q1 } \\
\text { Q2 } \\
\text { Q3 } \\
\text { Q4 }\end{array}$ & $\begin{array}{r}1208.8 \\
1178.5 \\
1001.3 \\
917.9\end{array}$ & $\begin{array}{l}676.2 \\
674.7 \\
636.4 \\
619.2\end{array}$ & $\begin{array}{l}146.7 \\
148.4 \\
148.5 \\
147.7\end{array}$ & $\begin{array}{ll}2 & 031.8 \\
2 & 001.6 \\
1 & 786.2 \\
1 & 684.8\end{array}$ & $\begin{array}{l}167.5 \\
164.5 \\
161.6 \\
162.4\end{array}$ & $\begin{array}{l}3798.3 \\
3742.0 \\
3551.6 \\
3596.5\end{array}$ & 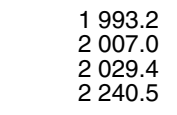 & $\begin{array}{l}-1805.1 \\
-1734.9 \\
-1522.1 \\
-1356.0\end{array}$ \\
\hline $\begin{array}{r}2009 \text { Q1 } \\
\text { Q2 } \\
\text { Q3 } \\
\text { Q4 }\end{array}$ & $\begin{array}{r}862.5 \\
940.0 \\
1119.9 \\
1212.6\end{array}$ & $\begin{array}{l}608.7 \\
623.3 \\
639.1 \\
633.5\end{array}$ & $\begin{array}{l}142.3 \\
142.3 \\
143.1 \\
143.6\end{array}$ & $\begin{array}{ll}1 & 613.4 \\
1 & 705.6 \\
1 & 902.2 \\
1 & 989.7\end{array}$ & $\begin{array}{l}165.7 \\
162.3 \\
162.1 \\
162.3\end{array}$ & $\begin{array}{l}3438.4 \\
3486.6 \\
3 \quad 789.2 \\
3839.1\end{array}$ & $\begin{array}{ll}2 & 168.1 \\
2 & 040.5 \\
2 & 119.1 \\
2 & 086.8\end{array}$ & $\begin{array}{ll}-1 & 270.3 \\
-1 & 446.1 \\
-1 & 670.0 \\
-1 & 752.3\end{array}$ \\
\hline $\begin{array}{r}2010 \text { Q1 } \\
\text { Q2 } \\
\text { Q3 } \\
\text { Q4 }\end{array}$ & $\begin{array}{ll}1 & 283.1 \\
1 & 120.4 \\
1 & 268.7 \\
1 & 373.7\end{array}$ & $\begin{array}{l}654.1^{\dagger} \\
628.8 \\
653.4 \\
675.3\end{array}$ & $\begin{array}{l}144.4^{\dagger} \\
144.5^{\dagger} \\
145.0 \\
144.9\end{array}$ & $\begin{array}{ll}2 & 081.5^{\dagger} \\
1 & 893.7 \\
2 & 067.1 \\
2 & 193.9\end{array}$ & $\begin{array}{l}170.3^{\dagger} \\
166.2 \\
165.1 \\
164.8\end{array}$ & $\begin{array}{l}4008.0^{\dagger} \\
3735.6 \\
3924.3 \\
4031.7\end{array}$ & $\begin{array}{l}2186.8^{\dagger} \\
2150.9 \\
2240.3 \\
2228.2\end{array}$ & $\begin{array}{l}-1821.2^{\dagger} \\
-1584.7 \\
-1684.0 \\
-1803.5\end{array}$ \\
\hline
\end{tabular}

1 Money market instruments

2 All loans secured on dwellings and all finance leasing are treated as long Sources: National Statistics term loans

3 Monetary financial institutions

4 Other than direct investment loans, loans secured on dwellings and loans for finance leasing

5 Reflects Housing Association reclassification in line with revisions policy back to 2005 Q1. 


\section{$12.1 \mathrm{C}^{\text {framatas }}$}

\begin{tabular}{|c|c|c|c|c|c|c|c|c|c|c|c|c|c|}
\hline & \multicolumn{13}{|c|}{ Financial assets } \\
\hline & \multicolumn{5}{|c|}{ Currency and deposits } & \multicolumn{8}{|c|}{ Securities other than shares } \\
\hline & \multirow{2}{*}{\multicolumn{3}{|c|}{$\begin{array}{r}\text { Deposits } \\
\text { with rest } \\
\text { of the } \\
\text { world } \\
\text { MFI's }\end{array}$}} & \multirow[b]{2}{*}{$\begin{array}{l}\text { Deposits } \\
\text { other than } \\
\text { with MFl's }\end{array}$} & \multirow[b]{2}{*}{ Total } & \multicolumn{3}{|c|}{ Short term MMl's ${ }^{2}$ issued } & \multicolumn{4}{|c|}{ Bonds issued } & \multirow[b]{2}{*}{ Total } \\
\hline & & & & & & $\begin{array}{r}\text { by UK } \\
\text { general } \\
\text { government }\end{array}$ & $\begin{array}{l}\text { by UK } \\
\text { MFl's }\end{array}$ & $\begin{array}{r}\text { by other } \\
\text { UK }\end{array}$ & $\begin{array}{r}\text { by UK } \\
\text { central } \\
\text { government }\end{array}$ & $\begin{array}{r}\text { by UK } \\
\text { local } \\
\text { authoriti- } \\
\text { es }\end{array}$ & $\begin{array}{r}\text { other by } \\
\text { UK } \\
\text { residents }\end{array}$ & $\begin{array}{l}\text { by rest of } \\
\text { the world }\end{array}$ & \\
\hline & $\mathrm{F} 21$ & $\mathrm{~F} 221$ & F229 & F29 & $\mathrm{F} 2$ & F3311 & F3315 & F3316 & F3321 & F3322 & F3326 & F3329 & F3 \\
\hline $\begin{array}{l}2004 \\
2005 \\
2006 \\
2007 \\
2008\end{array}$ & $\begin{array}{r}\text { NKDS } \\
0.6 \\
0.6 \\
0.6 \\
0.7 \\
0.6\end{array}$ & $\begin{array}{r}\text { NKDU } \\
4.9 \\
4.8 \\
6.0 \\
3.9 \\
2.6\end{array}$ & $\begin{array}{r}\text { NKDY } \\
- \\
- \\
- \\
- \\
-\end{array}$ & $\begin{array}{r}\text { NKDZ } \\
2.1 \\
3.5 \\
3.9 \\
3.9 \\
3.7\end{array}$ & $\begin{array}{r}\text { NKDR } \\
7.6 \\
9.0 \\
10.5 \\
8.5 \\
7.0\end{array}$ & $\begin{array}{r}\text { NKEC } \\
- \\
- \\
- \\
0.4 \\
0.4\end{array}$ & $\begin{array}{r}\text { NKEH } \\
0.4 \\
0.4 \\
0.4 \\
0.4 \\
0.4\end{array}$ & $\begin{array}{r}\text { NKEM } \\
1.3 \\
1.5 \\
1.9 \\
1.7 \\
0.9\end{array}$ & $\begin{array}{r}\text { NKEP } \\
3.3 \\
2.5 \\
0.5 \\
0.7 \\
1.2\end{array}$ & $\begin{array}{r}\text { NKES } \\
- \\
- \\
- \\
- \\
-\end{array}$ & $\begin{array}{r}\text { NKEW } \\
- \\
- \\
- \\
- \\
-\end{array}$ & $\begin{array}{r}\text { NKIQ } \\
- \\
- \\
- \\
- \\
-\end{array}$ & $\begin{array}{r}\text { NKEA } \\
4.9 \\
4.4 \\
2.8 \\
3.3 \\
2.9\end{array}$ \\
\hline $\begin{array}{r}2007 \text { Q2 } \\
\text { Q3 } \\
\text { Q4 }\end{array}$ & $\begin{array}{l}0.7 \\
0.6 \\
0.7\end{array}$ & $\begin{array}{l}4.4 \\
4.5 \\
3.9\end{array}$ & $\begin{array}{l}- \\
- \\
-\end{array}$ & $\begin{array}{l}3.5 \\
3.5 \\
3.9\end{array}$ & $\begin{array}{l}8.6 \\
8.5 \\
8.5\end{array}$ & $\begin{array}{r}- \\
\overline{-} \\
0 . \overline{4}\end{array}$ & $\begin{array}{l}0.4 \\
0.4 \\
0.4\end{array}$ & $\begin{array}{l}2.3 \\
1.9 \\
1.7\end{array}$ & $\begin{array}{l}0.5 \\
0.5 \\
0.7\end{array}$ & $\begin{array}{l}- \\
- \\
-\end{array}$ & $\begin{array}{l}- \\
- \\
-\end{array}$ & $\begin{array}{l}- \\
- \\
-\end{array}$ & $\begin{array}{l}3.3 \\
2.8 \\
3.3\end{array}$ \\
\hline $\begin{array}{r}2008 \text { Q1 } \\
\text { Q2 } \\
\text { Q3 } \\
\text { Q4 }\end{array}$ & $\begin{array}{l}0.6 \\
0.6 \\
0.6 \\
0.6\end{array}$ & $\begin{array}{l}3.1 \\
3.1 \\
3.0 \\
2.6\end{array}$ & $\begin{array}{l}- \\
- \\
- \\
-\end{array}$ & $\begin{array}{l}4.1 \\
4.4 \\
3.4 \\
3.7\end{array}$ & $\begin{array}{l}7.8 \\
8.0 \\
6.9 \\
7.0\end{array}$ & $\begin{array}{l}0.6 \\
0.8 \\
0.8 \\
0.4\end{array}$ & $\begin{array}{l}0.4 \\
0.4 \\
0.4 \\
0.4\end{array}$ & $\begin{array}{l}1.4 \\
1.3 \\
1.2 \\
0.9\end{array}$ & $\begin{array}{l}0.7 \\
0.8 \\
0.8 \\
1.2\end{array}$ & $\begin{array}{l}- \\
- \\
- \\
-\end{array}$ & $\begin{array}{l}- \\
- \\
- \\
-\end{array}$ & $\begin{array}{l}- \\
- \\
- \\
-\end{array}$ & $\begin{array}{l}3.2 \\
3.3 \\
3.2 \\
2.9\end{array}$ \\
\hline $\begin{array}{r}2009 \text { Q1 } \\
\text { Q2 } \\
\text { Q3 } \\
\text { Q4 }\end{array}$ & $\begin{array}{l}0.5 \\
0.5 \\
0.5 \\
0.6\end{array}$ & $\begin{array}{l}2.4 \\
2.5 \\
2.6 \\
2.8\end{array}$ & $\begin{array}{l}- \\
- \\
- \\
-\end{array}$ & $\begin{array}{l}3.7 \\
3.8 \\
3.5 \\
3.4\end{array}$ & $\begin{array}{l}6.6 \\
6.7 \\
6.6 \\
6.8\end{array}$ & $\begin{array}{l}0.3 \\
0.3 \\
0.3 \\
0.3\end{array}$ & $\begin{array}{l}0.4 \\
0.4 \\
0.4 \\
0.4\end{array}$ & $\begin{array}{l}0.7 \\
0.6 \\
0.7 \\
0.7\end{array}$ & $\begin{array}{l}1.3 \\
1.3 \\
1.3 \\
1.3\end{array}$ & $\begin{array}{l}- \\
- \\
- \\
-\end{array}$ & $\begin{array}{l}- \\
- \\
- \\
-\end{array}$ & $\begin{array}{l}0.1 \\
0.1 \\
0.1 \\
0.1\end{array}$ & $\begin{array}{l}2.8 \\
2.7 \\
2.8 \\
2.8\end{array}$ \\
\hline $\begin{array}{r}2010 \text { Q1 } \\
\text { Q2 } \\
\text { Q3 } \\
\text { Q4 }\end{array}$ & $\begin{array}{l}0.6 \\
0.5 \\
0.5 \\
0.6\end{array}$ & $\begin{array}{l}2.7 \\
2.6 \\
2.8 \\
2.7\end{array}$ & $\begin{array}{l}- \\
- \\
- \\
-\end{array}$ & $\begin{array}{l}2.7 \\
2.7 \\
2.7 \\
2.8\end{array}$ & $\begin{array}{l}6.0 \\
5.9 \\
6.1 \\
6.1\end{array}$ & $\begin{array}{l}0.3 \\
0.3 \\
0.3 \\
0.3\end{array}$ & $\begin{array}{l}0.4 \\
0.4 \\
0.4 \\
0.4\end{array}$ & $\begin{array}{l}0.7 \\
0.6 \\
0.7 \\
0.6\end{array}$ & $\begin{array}{l}1.3 \\
1.3 \\
1.3 \\
1.3\end{array}$ & $\begin{array}{l}- \\
- \\
- \\
-\end{array}$ & $\begin{array}{l}- \\
- \\
- \\
-\end{array}$ & $\begin{array}{l}0.1 \\
0.1 \\
0.1 \\
0.1\end{array}$ & $\begin{array}{l}2.8 \\
2.8 \\
2.8 \\
2.8\end{array}$ \\
\hline
\end{tabular}

\begin{tabular}{|c|c|c|c|c|c|c|c|c|c|c|c|c|}
\hline & \multicolumn{12}{|c|}{ Financial assets - continued } \\
\hline & \multicolumn{4}{|c|}{ Loans } & \multicolumn{5}{|c|}{ Shares and other equity } & \multirow{3}{*}{$\begin{array}{l}\text { Insurance } \\
\text { technical } \\
\text { reserves } \\
\text { Prepayments } \\
\text { of } \\
\text { insurance } \\
\text { premiums } \\
\text { etc. }\end{array}$} & \multirow{3}{*}{$\begin{array}{r}\text { Other } \\
\text { accounts } \\
\text { receivable- } \\
\text { /payable }\end{array}$} & \multirow[b]{3}{*}{$\begin{array}{r}\text { Total } \\
\text { financial } \\
\text { assets }\end{array}$} \\
\hline & \multicolumn{3}{|c|}{ Long term loans } & & \multicolumn{4}{|c|}{ Excl mutual funds' shares } & \multirow[b]{2}{*}{ Total } & & & \\
\hline & $\begin{array}{r}\text { direct } \\
\text { investment } \\
\text { loan }\end{array}$ & $\begin{array}{l}\text { Secured on } \\
\text { dwellings }\end{array}$ & $\begin{array}{l}\text { by UK } \\
\text { residents }{ }^{3}\end{array}$ & Total & $\begin{array}{r}\text { Quoted UK } \\
\text { shares }\end{array}$ & $\begin{array}{r}\text { Unquoted UK } \\
\text { shares }\end{array}$ & $\begin{array}{r}\text { by UK } \\
\text { residents }\end{array}$ & $\begin{array}{r}\text { By the rest } \\
\text { of the } \\
\text { world }\end{array}$ & & & & \\
\hline & F421 & F422 & $\mathrm{F} 424$ & $\mathrm{~F} 4$ & F514 & F515 & F517 & F519 & F5 & F62 & F7 & FA \\
\hline $\begin{array}{l}2004 \\
2005 \\
2006 \\
2007 \\
2008\end{array}$ & $\begin{array}{r}\text { ZYBN } \\
0.3 \\
0.3 \\
-\overline{1} \\
0.1 \\
0.1\end{array}$ & $\begin{array}{r}\text { NKFN } \\
- \\
- \\
- \\
- \\
-\end{array}$ & $\begin{array}{r}\text { NKFS } \\
4.0 \\
3.8 \\
2.7 \\
2.4 \\
2.5\end{array}$ & $\begin{array}{r}\text { NKFC } \\
4.4 \\
4.1 \\
2.7 \\
2.4 \\
2.6\end{array}$ & $\begin{array}{r}\text { NKFZ } \\
0.2 \\
- \\
- \\
- \\
-\end{array}$ & $\begin{array}{r}\text { NKGA } \\
0.3 \\
0.3 \\
0.3 \\
0.3 \\
0.3\end{array}$ & $\begin{array}{r}\text { NSOL } \\
- \\
- \\
- \\
- \\
-\end{array}$ & $\begin{array}{r}\text { NKGE } \\
1.6 \\
0.5 \\
0.5 \\
0.5 \\
0.3\end{array}$ & $\begin{array}{r}\text { NKFU } \\
2.1 \\
0.8 \\
0.8 \\
0.8 \\
0.6\end{array}$ & $\begin{array}{r}\text { NKGN } \\
- \\
- \\
- \\
- \\
-\end{array}$ & $\begin{array}{r}\text { NKGO } \\
10.9 \\
11.1 \\
16.2 \\
17.8 \\
17.4\end{array}$ & $\begin{array}{r}\text { NKFB } \\
29.9 \\
29.3 \\
33.0 \\
32.8 \\
30.5\end{array}$ \\
\hline $\begin{array}{l}2009 \\
2010\end{array}$ & $\begin{array}{l}- \\
-\end{array}$ & $\begin{array}{l}- \\
-\end{array}$ & $\begin{array}{l}2.1 \\
2.3\end{array}$ & $\begin{array}{l}2.1 \\
2.3\end{array}$ & $\begin{array}{l}- \\
-\end{array}$ & $\begin{array}{l}0.3 \\
0.3\end{array}$ & $\begin{array}{l}- \\
-\end{array}$ & $\begin{array}{l}0.4 \\
0.6\end{array}$ & $\begin{array}{l}0.8 \\
0.9\end{array}$ & $\begin{array}{l}- \\
-\end{array}$ & $\begin{array}{l}19.5 \\
20.3\end{array}$ & $\begin{array}{l}31.9 \\
32.3\end{array}$ \\
\hline $\begin{array}{r}2007 \text { Q2 } \\
\text { Q3 } \\
\text { Q4 }\end{array}$ & $\begin{array}{l}-\overline{1} \\
0.1\end{array}$ & $\begin{array}{l}- \\
- \\
-\end{array}$ & $\begin{array}{l}2.5 \\
2.5 \\
2.4\end{array}$ & $\begin{array}{l}2.6 \\
2.5 \\
2.4\end{array}$ & $\begin{array}{l}- \\
- \\
-\end{array}$ & $\begin{array}{l}0.3 \\
0.3 \\
0.3\end{array}$ & $\begin{array}{l}- \\
- \\
-\end{array}$ & $\begin{array}{l}0.4 \\
0.4 \\
0.5\end{array}$ & $\begin{array}{l}0.7 \\
0.7 \\
0.8\end{array}$ & $\begin{array}{l}- \\
- \\
-\end{array}$ & $\begin{array}{l}17.0 \\
17.3 \\
17.8\end{array}$ & $\begin{array}{l}32.1 \\
31.9 \\
32.8\end{array}$ \\
\hline $\begin{array}{r}2008 \text { Q1 } \\
\text { Q2 } \\
\text { Q3 } \\
\text { Q4 }\end{array}$ & $\begin{array}{r}-0.1 \\
0.1 \\
-\overline{1} \\
0.1\end{array}$ & $\begin{array}{l}- \\
- \\
- \\
-\end{array}$ & $\begin{array}{l}2.2 \\
2.5 \\
2.5 \\
2.5\end{array}$ & $\begin{array}{l}2.1 \\
2.7 \\
2.5 \\
2.6\end{array}$ & $\begin{array}{l}- \\
- \\
- \\
-\end{array}$ & $\begin{array}{l}0.3 \\
0.3 \\
0.3 \\
0.3\end{array}$ & $\begin{array}{l}- \\
- \\
- \\
-\end{array}$ & $\begin{array}{l}0.4 \\
0.4 \\
0.3 \\
0.3\end{array}$ & $\begin{array}{l}0.7 \\
0.7 \\
0.6 \\
0.6\end{array}$ & $\begin{array}{l}- \\
- \\
- \\
-\end{array}$ & $\begin{array}{l}18.4 \\
17.7 \\
17.6 \\
17.4\end{array}$ & $\begin{array}{l}32.2 \\
32.3 \\
30.8 \\
30.5\end{array}$ \\
\hline $\begin{array}{r}2009 \text { Q1 } \\
\text { Q2 } \\
\text { Q3 } \\
\text { Q4 }\end{array}$ & $\begin{array}{l}- \\
- \\
- \\
-\end{array}$ & $\begin{array}{l}- \\
- \\
- \\
-\end{array}$ & $\begin{array}{l}2.2 \\
2.1 \\
2.1 \\
2.1\end{array}$ & $\begin{array}{l}2.2 \\
2.1 \\
2.2 \\
2.1\end{array}$ & $\begin{array}{l}- \\
- \\
- \\
-\end{array}$ & $\begin{array}{l}0.3 \\
0.3 \\
0.3 \\
0.3\end{array}$ & $\begin{array}{l}- \\
- \\
- \\
-\end{array}$ & $\begin{array}{l}0.4 \\
0.4 \\
0.4 \\
0.4\end{array}$ & $\begin{array}{l}0.7 \\
0.7 \\
0.7 \\
0.8\end{array}$ & $\begin{array}{l}- \\
- \\
- \\
-\end{array}$ & $\begin{array}{l}17.0 \\
18.5 \\
19.3 \\
19.5\end{array}$ & $\begin{array}{l}29.3 \\
30.8 \\
31.6 \\
31.9\end{array}$ \\
\hline $\begin{array}{r}2010 \text { Q1 } \\
\text { Q2 } \\
\text { Q3 } \\
\text { Q4 }\end{array}$ & $\begin{array}{l}- \\
- \\
- \\
-\end{array}$ & $\begin{array}{l}- \\
- \\
- \\
-\end{array}$ & $\begin{array}{l}2.2 \\
2.2 \\
2.1 \\
2.3\end{array}$ & $\begin{array}{l}2.2 \\
2.2 \\
2.1 \\
2.3\end{array}$ & $\begin{array}{l}- \\
- \\
- \\
-\end{array}$ & $\begin{array}{l}0.3 \\
0.3 \\
0.3 \\
0.3\end{array}$ & $\begin{array}{l}- \\
- \\
- \\
-\end{array}$ & $\begin{array}{l}0.5 \\
0.5 \\
0.5 \\
0.6\end{array}$ & $\begin{array}{l}0.8 \\
0.8 \\
0.8 \\
0.9\end{array}$ & $\begin{array}{l}- \\
- \\
- \\
-\end{array}$ & $\begin{array}{l}19.7^{\dagger} \\
20.0^{\dagger} \\
20.4 \\
20.3\end{array}$ & $\begin{array}{l}31.5 \\
31.6^{\dagger} \\
32.3 \\
32.3\end{array}$ \\
\hline $\begin{array}{l}1 \text { Monet } \\
2 \text { Money } \\
3 \text { Other } \\
\text { for fina }\end{array}$ & $\begin{array}{l}\text { inancial inst } \\
\text { rket instrum } \\
\text { direct inve } \\
\text { leasing }\end{array}$ & $\begin{array}{l}\text { itutions } \\
\text { ents } \\
\text { stment loans, }\end{array}$ & ans secure & $n_{0}$ & inge and lo & & & & & & $\begin{array}{r}\text { es: National } \\
\text { Bank of }\end{array}$ & $\begin{array}{l}\text { Statistics; } \\
\text { England }\end{array}$ \\
\hline
\end{tabular}




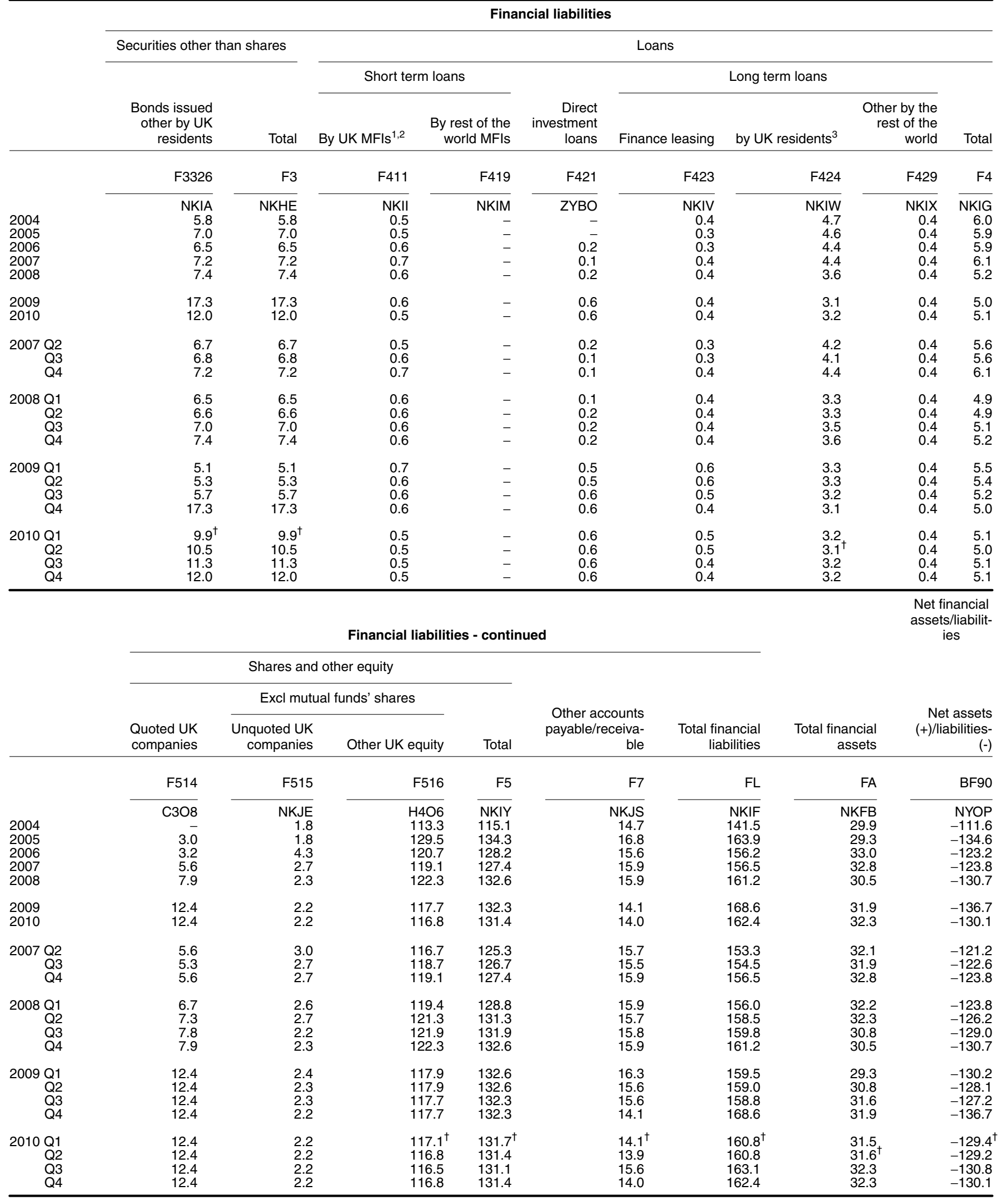

1 All loans secured on dwellings and all finance leasing are treated as long

Sources: National Statistics toans

2 Monetary financial institutions

3 Other than direct investment loans, loans secured on dwellings and loans

for financial leasing 


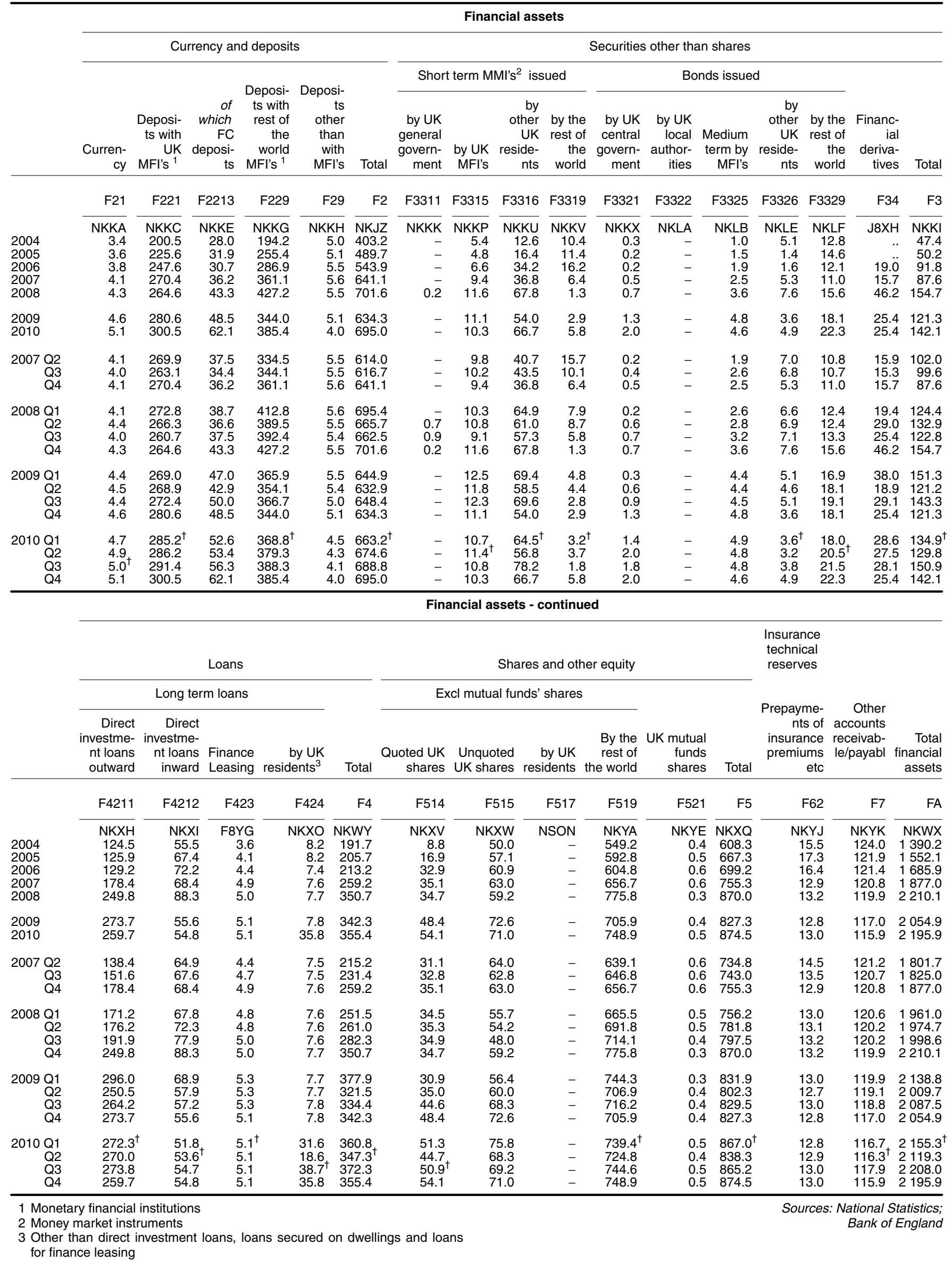




\begin{tabular}{|c|c|c|c|c|c|c|c|c|c|c|c|c|c|c|c|}
\hline & \multicolumn{15}{|c|}{ Financial liabilities } \\
\hline & \multicolumn{4}{|c|}{ Securities other than shares } & \multicolumn{11}{|c|}{ Loans } \\
\hline & \multirow[b]{2}{*}{$\begin{array}{c}\text { MMl's }^{1} \\
\text { issued by } \\
\text { other UK } \\
\text { residents }\end{array}$} & \multirow[b]{2}{*}{$\begin{array}{r}\text { Bonds } \\
\text { issued } \\
\text { other by } \\
\text { UK } \\
\text { residents }\end{array}$} & \multirow[b]{2}{*}{$\begin{array}{r}\text { Financial } \\
\text { derivati- } \\
\text { ves }\end{array}$} & \multirow[b]{2}{*}{ Total } & \multicolumn{3}{|c|}{ Short term loans } & \multicolumn{7}{|c|}{ Long term loans } & \multirow[b]{2}{*}{ Total } \\
\hline & & & & & $\begin{array}{l}\text { By UK } \\
\text { MFIs }^{2,3}\end{array}$ & $\begin{array}{r}\text { of which } \\
\text { FC loans } \\
\text { by UK } \\
\text { MFIs }\end{array}$ & $\begin{array}{c}\text { by rest } \\
\text { of the } \\
\text { world } \\
\text { MFIs }\end{array}$ & $\begin{array}{c}\text { Direct } \\
\text { investme- } \\
\text { nt loans } \\
\text { outward }\end{array}$ & $\begin{array}{r}\text { Direct } \\
\text { investme- } \\
\text { nt loans } \\
\text { inward }\end{array}$ & $\begin{array}{r}\text { Secured } \\
\text { on } \\
\text { dwellings } \\
55\end{array}$ & $\begin{array}{l}\text { of which } \\
\text { by other, } \\
\text { non-MFl's }\end{array}$ & $\begin{array}{r}\text { Finance } \\
\text { leasing }\end{array}$ & $\begin{array}{r}\text { by UK } \\
\text { residents }^{4}\end{array}$ & $\begin{array}{r}\text { Other by } \\
\text { the rest } \\
\text { of the } \\
\text { world }\end{array}$ & \\
\hline & F3316 & F3326 & F34 & F3 & $\mathrm{F} 411$ & F4112 & F419 & F4211 & F4212 & $\mathrm{F} 422$ & F4229 & $\mathrm{F} 423$ & $\mathrm{~F} 424$ & F429 & $\mathrm{F} 4$ \\
\hline $\begin{array}{l}2004 \\
2005 \\
2006 \\
2007 \\
2008\end{array}$ & $\begin{array}{r}\text { NKZM } \\
21.8 \\
23.8 \\
21.7 \\
22.2 \\
31.1\end{array}$ & $\begin{array}{r}\text { NKZW } \\
254.7 \\
301.2 \\
335.6 \\
332.5 \\
320.3\end{array}$ & $\begin{array}{r}\text { J8XI } \\
. . \\
. . \\
22.5 \\
21.0 \\
51.1\end{array}$ & $\begin{array}{l}\text { NKZA } \\
276.5 \\
325.0 \\
379.7 \\
375.8 \\
402.5\end{array}$ & $\begin{array}{l}\text { NLBE } \\
298.5 \\
346.0 \\
397.0 \\
476.9 \\
538.2\end{array}$ & $\begin{array}{r}\text { NLBG } \\
31.8 \\
42.4 \\
43.1 \\
66.9 \\
97.3\end{array}$ & $\begin{array}{r}\text { NLBI } \\
158.5 \\
166.0 \\
220.6 \\
208.8 \\
263.2\end{array}$ & $\begin{array}{l}\text { NLBL } \\
125.6 \\
159.2 \\
168.6 \\
152.9 \\
227.4\end{array}$ & $\begin{array}{r}\text { NLBM } \\
125.0 \\
149.3 \\
156.6 \\
129.5 \\
149.6\end{array}$ & $\begin{array}{r}\text { G9JM } \\
-\overline{-} \\
27.1 \\
30.9 \\
34.8 \\
40.2\end{array}$ & $\begin{array}{r}\text { G9JL } \\
3 . \overline{8} \\
4.5 \\
4.1 \\
5.2\end{array}$ & $\begin{array}{r}\text { NLBR } \\
22.5 \\
22.9 \\
23.4 \\
23.8 \\
24.1\end{array}$ & $\begin{array}{r}\text { NLBS } \\
78.9 \\
72.9 \\
91.3 \\
107.1 \\
90.8\end{array}$ & $\begin{array}{r}\text { NLBT } \\
0.4 \\
0.4 \\
0.4 \\
0.4 \\
0.5\end{array}$ & $\begin{array}{r}\text { NLBC } \\
809.4 \\
943.8 \\
1088.7 \\
1134.2 \\
1334.1\end{array}$ \\
\hline $\begin{array}{l}2009 \\
2010\end{array}$ & $\begin{array}{l}22.5 \\
27.7\end{array}$ & $\begin{array}{l}375.5 \\
337.2\end{array}$ & $\begin{array}{l}33.3 \\
35.5\end{array}$ & $\begin{array}{l}431.3 \\
400.4\end{array}$ & $\begin{array}{l}478.5 \\
440.4\end{array}$ & $\begin{array}{l}59.9 \\
54.4\end{array}$ & $\begin{array}{l}205.8 \\
250.7\end{array}$ & $\begin{array}{l}228.4 \\
243.7\end{array}$ & $\begin{array}{l}138.0 \\
135.2\end{array}$ & $\begin{array}{l}43.5 \\
40.7\end{array}$ & $\begin{array}{l}1.8 \\
1.7\end{array}$ & $\begin{array}{l}24.5 \\
24.8\end{array}$ & $\begin{array}{l}114.4 \\
119.7\end{array}$ & $\begin{array}{l}0.5 \\
0.5\end{array}$ & $\begin{array}{l}1233.4 \\
1255.6\end{array}$ \\
\hline $\begin{array}{r}2007 \text { Q2 } \\
\text { Q3 } \\
\text { Q4 }\end{array}$ & $\begin{array}{l}19.0 \\
21.1 \\
22.2\end{array}$ & $\begin{array}{l}313.0 \\
313.9 \\
332.5\end{array}$ & $\begin{array}{l}16.0 \\
18.7 \\
21.0\end{array}$ & $\begin{array}{l}348.0 \\
353.7 \\
375.8\end{array}$ & $\begin{array}{l}423.9 \\
454.8 \\
476.9\end{array}$ & $\begin{array}{l}44.4 \\
52.9 \\
66.9\end{array}$ & $\begin{array}{l}227.5 \\
209.3 \\
208.8\end{array}$ & $\begin{array}{l}151.5 \\
157.6 \\
152.9\end{array}$ & $\begin{array}{l}129.1 \\
129.2 \\
129.5\end{array}$ & $\begin{array}{l}32.4 \\
33.2 \\
34.8\end{array}$ & $\begin{array}{l}4.2 \\
4.2 \\
4.1\end{array}$ & $\begin{array}{l}23.6 \\
23.7 \\
23.8\end{array}$ & $\begin{array}{l}100.1 \\
107.4 \\
107.1\end{array}$ & $\begin{array}{l}0.4 \\
0.4 \\
0.4\end{array}$ & $\begin{array}{ll}1 & 088.5 \\
1 & 115.8 \\
1 & 134.2\end{array}$ \\
\hline $\begin{array}{r}2008 \text { Q1 } \\
\text { Q2 } \\
\text { Q3 } \\
\text { Q4 }\end{array}$ & $\begin{array}{l}21.4 \\
21.6 \\
23.7 \\
31.1\end{array}$ & $\begin{array}{l}327.5 \\
305.1 \\
327.6 \\
320.3\end{array}$ & $\begin{array}{l}23.9 \\
31.3 \\
33.0 \\
51.1\end{array}$ & $\begin{array}{l}372.7 \\
358.1 \\
384.3 \\
402.5\end{array}$ & $\begin{array}{l}508.0 \\
512.6 \\
525.5 \\
538.2\end{array}$ & $\begin{array}{l}78.4 \\
74.0 \\
79.3 \\
97.3\end{array}$ & $\begin{array}{l}235.6 \\
222.9 \\
200.7 \\
263.2\end{array}$ & $\begin{array}{l}168.3 \\
168.5 \\
185.9 \\
227.4\end{array}$ & $\begin{array}{l}139.3 \\
140.0 \\
138.7 \\
149.6\end{array}$ & $\begin{array}{l}36.2 \\
37.3 \\
38.5 \\
40.2\end{array}$ & $\begin{array}{l}4.1 \\
4.1 \\
4.0 \\
5.2\end{array}$ & $\begin{array}{l}23.9 \\
24.0 \\
24.1 \\
24.1\end{array}$ & $\begin{array}{r}103.2 \\
100.6 \\
93.5 \\
90.8\end{array}$ & $\begin{array}{l}0.4 \\
0.4 \\
0.4 \\
0.5\end{array}$ & $\begin{array}{ll}1 & 214.8 \\
1 & 206.3 \\
1 & 207.4 \\
1 & 334.1\end{array}$ \\
\hline $\begin{array}{r}2009 \text { Q1 } \\
\text { Q2 } \\
\text { Q3 } \\
\text { Q4 }\end{array}$ & $\begin{array}{l}29.8 \\
25.4 \\
26.1 \\
22.5\end{array}$ & $\begin{array}{l}251.3 \\
303.9 \\
369.0 \\
375.5\end{array}$ & $\begin{array}{l}45.3 \\
30.6 \\
39.1 \\
33.3\end{array}$ & $\begin{array}{l}326.4 \\
359.8 \\
434.2 \\
431.3\end{array}$ & $\begin{array}{l}537.2 \\
511.4 \\
491.6 \\
478.5\end{array}$ & $\begin{array}{l}92.6 \\
75.3 \\
66.2 \\
59.9\end{array}$ & $\begin{array}{l}221.6 \\
197.2 \\
208.9 \\
205.8\end{array}$ & $\begin{array}{l}245.1 \\
206.7 \\
227.8 \\
228.4\end{array}$ & $\begin{array}{l}147.9 \\
136.9 \\
140.2 \\
138.0\end{array}$ & $\begin{array}{l}41.2 \\
41.8 \\
42.5 \\
43.5\end{array}$ & $\begin{array}{l}5.2 \\
1.8 \\
1.8 \\
1.8\end{array}$ & $\begin{array}{l}24.2 \\
24.3 \\
24.4 \\
24.5\end{array}$ & $\begin{array}{l}104.6 \\
129.4 \\
144.0 \\
114.4\end{array}$ & $\begin{array}{l}0.5 \\
0.5 \\
0.5 \\
0.5\end{array}$ & $\begin{array}{ll}1 & 322.3 \\
1 & 248.1 \\
1 & 279.8 \\
1 & 233.4\end{array}$ \\
\hline $\begin{array}{r}2010 \text { Q1 } \\
\text { Q2 } \\
\text { Q3 } \\
\text { Q4 }\end{array}$ & $\begin{array}{l}27.0 \\
29.8 \\
28.9 \\
27.7\end{array}$ & $\begin{array}{l}382.5 \\
333.7^{\dagger} \\
312.1 \\
337.2\end{array}$ & $\begin{array}{l}37.9 \\
41.3 \\
38.0 \\
35.5\end{array}$ & $\begin{array}{l}447.4 \\
404.7^{\dagger} \\
379.0 \\
400.4\end{array}$ & $\begin{array}{l}480.5 \\
470.4 \\
462.4^{\dagger} \\
440.4\end{array}$ & $\begin{array}{l}60.7 \\
57.0^{\dagger} \\
56.8^{\dagger} \\
54.4\end{array}$ & $\begin{array}{l}220.0^{\dagger} \\
217.4 \\
248.7 \\
250.7\end{array}$ & $\begin{array}{l}235.9^{\dagger} \\
241.0 \\
249.0 \\
243.7\end{array}$ & $\begin{array}{l}138.3^{\dagger} \\
132.8 \\
132.7 \\
135.2\end{array}$ & $\begin{array}{l}43.3 \\
43.9 \\
44.1 \\
40.7\end{array}$ & $\begin{array}{l}1.7 \\
1.7 \\
1.7 \\
1.7\end{array}$ & $\begin{array}{l}24.6 \\
24.7 \\
24.7 \\
24.8\end{array}$ & $\begin{array}{l}150.6^{\dagger} \\
124.8 \\
134.7 \\
119.7\end{array}$ & $\begin{array}{l}0.5 \\
0.5 \\
0.5 \\
0.5\end{array}$ & $\begin{array}{ll}1 & 293.7^{\dagger} \\
1 & 255.4 \\
1 & 296.8 \\
1 & 255.6\end{array}$ \\
\hline
\end{tabular}

Financial liabilities - continued

Shares and other equity

Excl mutual funds' shares

\begin{tabular}{|c|c|c|c|c|c|c|c|c|c|}
\hline & $\begin{array}{l}\text { Quoted UK } \\
\text { companies }\end{array}$ & $\begin{array}{r}\text { Unquoted UK } \\
\text { companies }\end{array}$ & Other UK equity & by UK residents & Total & $\begin{array}{r}\text { Other accounts } \\
\text { receivable/ } \\
\text { payable }\end{array}$ & $\begin{array}{r}\text { Total financial } \\
\text { liabilities }\end{array}$ & $\begin{array}{r}\text { Total financial } \\
\text { assets }\end{array}$ & $\begin{array}{r}\text { Net assets } \\
(+) / \text { /iabilitie- } \\
\text { s(-) }\end{array}$ \\
\hline & F514 & F515 & F516 & F517 & F5 & $\mathrm{F} 7$ & $\mathrm{FL}$ & FA & BF90 \\
\hline $\begin{array}{l}2004 \\
2005 \\
2006 \\
2007 \\
2008\end{array}$ & $\begin{array}{rr} & \text { NLBZ } \\
1 & 080.2 \\
1 & 232.3 \\
1 & 315.5 \\
1 & 360.5 \\
910.0\end{array}$ & $\begin{array}{l}\text { NLCA } \\
422.1 \\
513.2 \\
606.8 \\
669.9 \\
616.9\end{array}$ & $\begin{array}{r}\text { NLCB } \\
19.2 \\
19.7 \\
21.6 \\
26.2 \\
25.4\end{array}$ & $\begin{array}{r}\text { NSOO } \\
- \\
- \\
- \\
- \\
-\end{array}$ & $\begin{array}{r}\text { NLBU } \\
1521.5 \\
1765.3 \\
1943.9 \\
2056.6 \\
1552.2\end{array}$ & $\begin{array}{r}\text { NLCO } \\
140.0 \\
142.7 \\
146.8 \\
147.2 \\
146.5\end{array}$ & $\begin{array}{r}\text { NLBB } \\
2747.4 \\
3176.8 \\
3559.2 \\
3713.7 \\
3435.3\end{array}$ & $\begin{array}{r}\text { NKWX } \\
1390.2 \\
1552.1 \\
1685.9 \\
1877.0 \\
2210.1\end{array}$ & $\begin{array}{r}\text { NYOT } \\
-1357.2 \\
-1624.7 \\
-1873.2 \\
-1836.7 \\
-1225.3\end{array}$ \\
\hline $\begin{array}{l}2009 \\
2010\end{array}$ & $\begin{array}{l}1200.3 \\
1361.3\end{array}$ & $\begin{array}{l}631.2 \\
673.1\end{array}$ & $\begin{array}{l}25.9 \\
28.0\end{array}$ & $\begin{array}{l}- \\
-\end{array}$ & $\begin{array}{l}1857.4 \\
2062.5\end{array}$ & $\begin{array}{l}148.3 \\
150.8\end{array}$ & $\begin{array}{l}3670.5 \\
3869.3\end{array}$ & $\begin{array}{l}2054.9 \\
2195.9\end{array}$ & $\begin{array}{l}-1615.6 \\
-1673.4\end{array}$ \\
\hline $\begin{array}{r}2007 \text { Q2 } \\
\text { Q3 } \\
\text { Q4 }\end{array}$ & $\begin{array}{l}1380.7 \\
1356.5 \\
1360.5\end{array}$ & $\begin{array}{l}639.9 \\
659.4 \\
669.9\end{array}$ & $\begin{array}{l}24.8 \\
25.9 \\
26.2\end{array}$ & $\begin{array}{l}- \\
- \\
-\end{array}$ & $\begin{array}{ll}2 & 045.3 \\
2 & 041.9 \\
2 & 056.6\end{array}$ & $\begin{array}{l}148.1 \\
146.6 \\
147.2\end{array}$ & $\begin{array}{l}3629.9 \\
3658.0 \\
3713.7\end{array}$ & $\begin{array}{l}1801.7 \\
1825.0 \\
1877.0\end{array}$ & $\begin{array}{l}-1828.2 \\
-1833.0 \\
-1836.7\end{array}$ \\
\hline $\begin{array}{r}2008 \text { Q1 } \\
\text { Q2 } \\
\text { Q3 } \\
\text { Q4 }\end{array}$ & $\begin{array}{r}1202.1 \\
1171.2 \\
993.5 \\
910.0\end{array}$ & $\begin{array}{l}673.6 \\
672.0 \\
634.2 \\
616.9\end{array}$ & $\begin{array}{l}27.3 \\
27.1 \\
26.7 \\
25.4\end{array}$ & $\begin{array}{l}- \\
- \\
- \\
-\end{array}$ & $\begin{array}{l}1903.0 \\
1870.2 \\
1654.3 \\
1552.2\end{array}$ & $\begin{array}{l}151.7 \\
148.9 \\
145.8 \\
146.5\end{array}$ & $\begin{array}{l}3642.3 \\
3583.5 \\
3531.8 \\
3535.3\end{array}$ & $\begin{array}{ll}1 & 961.0 \\
1 & 974.7 \\
1 & 998.6 \\
2 & 210.1\end{array}$ & $\begin{array}{l}-1681.3 \\
-1608.8 \\
-1393.1 \\
-1225.3\end{array}$ \\
\hline $\begin{array}{r}2009 \text { Q1 } \\
\text { Q2 } \\
\text { Q3 } \\
\text { Q4 }\end{array}$ & $\begin{array}{r}850.1 \\
927.7 \\
1107.6 \\
1200.3\end{array}$ & $\begin{array}{l}606.3 \\
620.9 \\
636.8 \\
631.2\end{array}$ & $\begin{array}{l}24.4 \\
24.4 \\
25.4 \\
25.9\end{array}$ & $\begin{array}{l}- \\
- \\
- \\
-\end{array}$ & $\begin{array}{ll}1 & 480.9 \\
1 & 573.0 \\
1 & 769.8 \\
1 & 857.4\end{array}$ & $\begin{array}{l}149.4 \\
146.6 \\
146.5 \\
148.3\end{array}$ & $\begin{array}{l}3278.9 \\
3527.6 \\
3630.4 \\
3670.5\end{array}$ & $\begin{array}{ll}2 & 138.8 \\
2 & 009.7 \\
2 & 087.5 \\
2 & 054.9\end{array}$ & $\begin{array}{ll}-1 & 140.1 \\
-1 & 317.9 \\
-1 & 542.8 \\
-1 & 615.6\end{array}$ \\
\hline $\begin{array}{r}2010 \text { Q1 } \\
\text { Q2 } \\
\text { Q3 } \\
\text { Q4 }\end{array}$ & $\begin{array}{ll}1 & 270.7 \\
1 & 108.1 \\
1 & 256.3 \\
1 & 361.3\end{array}$ & $\begin{array}{l}651.9^{\dagger} \\
626.6 \\
651.2 \\
673.1\end{array}$ & $\begin{array}{l}27.2 \\
27.7 \\
28.4 \\
28.0\end{array}$ & $\begin{array}{l}- \\
- \\
- \\
-\end{array}$ & $\begin{array}{ll}1 & 949.9^{\dagger} \\
1 & 762.3 \\
1 & 936.0 \\
2 & 062.5\end{array}$ & $\begin{array}{l}156.1^{\dagger} \\
152.3 \\
149.5 \\
150.8\end{array}$ & $\begin{array}{l}3847.2^{\dagger} \\
3574.7 \\
3761.2 \\
3869.3\end{array}$ & $\begin{array}{l}2155.3^{\dagger} \\
2119.3 \\
2208.0 \\
2195.9\end{array}$ & $\begin{array}{l}-1691.8^{\dagger} \\
-1455.5 \\
-1553.2 \\
-1673.4\end{array}$ \\
\hline
\end{tabular}

1 Money market instruments

2 All loans secured on dwellings and all finance leasing are treated as long

Sources: National Statistics; term loans

3 Monetary financial institutions

4 Other than direct investment loans, loans secured on dwellings and loans

for finance leasing

5 Reflects Housing Association reclassification in line with revisions policy back to $2005 \mathrm{Q} 1$. 


\begin{tabular}{|c|c|c|c|c|c|c|c|c|c|c|c|c|c|c|c|c|c|}
\hline & \multicolumn{17}{|c|}{ Financial assets } \\
\hline & \multicolumn{5}{|c|}{ Currency and deposits } & \multicolumn{12}{|c|}{ Securities other than shares } \\
\hline & \multirow[b]{2}{*}{$\begin{array}{r}\text { Curren- } \\
\text { cy }\end{array}$} & \multirow[b]{2}{*}{$\begin{array}{r}\text { Deposi- } \\
\text { ts with } \\
\text { UK } \\
\text { MFI's }^{1}\end{array}$} & \multirow[b]{2}{*}{$\begin{array}{l}\text { Deposi- } \\
\text { ts with } \\
\text { rest of } \\
\text { the } \\
\text { world } \\
\text { MFl's }\end{array}$} & \multirow[b]{2}{*}{$\begin{array}{r}\text { Deposi- } \\
\text { ts } \\
\text { other } \\
\text { than } \\
\text { with } \\
\text { MFl's }\end{array}$} & \multirow[b]{2}{*}{ Total } & \multicolumn{5}{|c|}{ Short term MMl's ${ }^{2}$ issued } & \multicolumn{5}{|c|}{ Bonds issued } & \multirow[b]{2}{*}{$\begin{array}{r}\text { Financ- } \\
\text { ial } \\
\text { deriva- } \\
\text { tives }\end{array}$} & \multirow[b]{2}{*}{ Total } \\
\hline & & & & & & $\begin{array}{r}\text { by UK } \\
\text { general } \\
\text { govern- } \\
\text { ment }\end{array}$ & $\begin{array}{r}\text { UK } \\
\text { local } \\
\text { author- } \\
\text { ity } \\
\text { bills }\end{array}$ & $\begin{array}{l}\text { by UK } \\
\text { MFI's }\end{array}$ & $\begin{array}{r}\text { by } \\
\text { other } \\
\text { UK } \\
\text { reside- } \\
\text { nts }\end{array}$ & $\begin{array}{l}\text { by the } \\
\text { rest of } \\
\text { the } \\
\text { world }\end{array}$ & $\begin{array}{r}\text { by UK } \\
\text { central } \\
\text { govern- } \\
\text { ment }\end{array}$ & $\begin{array}{r}\text { by UK } \\
\text { local } \\
\text { author- } \\
\text { ities }\end{array}$ & $\begin{array}{r}\text { Medium } \\
\text { term by } \\
\text { MFl's }\end{array}$ & $\begin{array}{r}\text { Other } \\
\text { by UK } \\
\text { reside- } \\
\text { nts }\end{array}$ & $\begin{array}{l}\text { by the } \\
\text { rest of } \\
\text { the } \\
\text { world }\end{array}$ & & \\
\hline & $\mathrm{F} 21$ & F221 & F229 & $\mathrm{F} 29$ & $\mathrm{~F} 2$ & F3311 & F3312 & F3315 & F3316 & F3319 & F3321 & F3322 & F3325 & F3326 & F3329 & F34 & F3 \\
\hline $\begin{array}{l}2004 \\
2005 \\
2006 \\
2007 \\
2008\end{array}$ & $\begin{array}{r}\text { NLJE } \\
11.3 \\
10.2 \\
10.1 \\
8.7 \\
8.1\end{array}$ & $\begin{array}{rr} & \text { NLJG } \\
1 & 180.6 \\
1 & 446.1 \\
1 & 833.6 \\
1 & 533.5 \\
1 & 888.6\end{array}$ & $\begin{array}{rr} & \text { NLJK } \\
1 & 357.0 \\
1 & 740.4 \\
1 & 838.1 \\
2 & 330.3 \\
2 & 527.5\end{array}$ & $\begin{array}{r}\text { NLJL } \\
2.2 \\
3.6 \\
1.5 \\
3.8 \\
11.5\end{array}$ & $\begin{array}{rr} & \text { NLJD } \\
2 & 551.1 \\
3 & 200.4 \\
3 & 683.3 \\
3 & 876.2 \\
4 & 435.7\end{array}$ & $\begin{array}{r}\text { NLJO } \\
21.1 \\
18.2 \\
15.7 \\
10.3 \\
9.8\end{array}$ & $\begin{array}{r}\text { NLJS } \\
- \\
- \\
- \\
- \\
-\end{array}$ & $\begin{array}{l}\text { NLJT } \\
139.8 \\
144.1 \\
150.4 \\
148.1 \\
135.9\end{array}$ & $\begin{array}{r}\text { NLJY } \\
5.1 \\
9.4 \\
10.3 \\
9.4 \\
5.1\end{array}$ & $\begin{array}{r}\text { NLJZ } \\
47.6 \\
50.6 \\
56.3 \\
64.5 \\
65.5\end{array}$ & $\begin{array}{l}\text { NLKB } \\
254.3 \\
273.1 \\
296.9 \\
319.1 \\
401.5\end{array}$ & $\begin{array}{r}\text { NLKE } \\
0.4 \\
0.4 \\
0.5 \\
0.3 \\
0.2\end{array}$ & $\begin{array}{r}\text { NLKF } \\
62.8 \\
78.5 \\
89.0 \\
115.9 \\
153.8\end{array}$ & $\begin{array}{l}\text { NLKI } \\
345.0 \\
409.8 \\
464.6 \\
463.9 \\
446.1\end{array}$ & $\begin{array}{r}\text { NLKJ } \\
573.7 \\
677.4 \\
763.4 \\
866.6 \\
1026.9\end{array}$ & $\begin{array}{r}\text { NLKK } \\
- \\
1706 . \overline{9} \\
2805.1 \\
9566.2\end{array}$ & $\begin{array}{r}\text { NLJM } \\
1449.8 \\
1661.6 \\
3553.9 \\
4803.2 \\
11811.1\end{array}$ \\
\hline $\begin{array}{l}2009 \\
2010\end{array}$ & $\begin{array}{l}12.8 \\
10.4\end{array}$ & $\begin{array}{l}2141.5 \\
2013.5\end{array}$ & $\begin{array}{l}2187.3 \\
2365.1\end{array}$ & $\begin{array}{l}16.9 \\
10.6\end{array}$ & $\begin{array}{l}4358.5 \\
4399.6\end{array}$ & $\begin{array}{l}37.6 \\
26.5\end{array}$ & $\begin{array}{l}- \\
-\end{array}$ & $\begin{array}{l}73.5 \\
56.2\end{array}$ & $\begin{array}{l}4.3 \\
3.0\end{array}$ & $\begin{array}{l}75.9 \\
76.8\end{array}$ & $\begin{array}{l}560.6 \\
671.1\end{array}$ & $\begin{array}{l}0.5 \\
0.6\end{array}$ & $\begin{array}{l}176.4 \\
176.1\end{array}$ & $\begin{array}{l}582.9 \\
583.2\end{array}$ & $\begin{array}{l}1128.6 \\
1242.4\end{array}$ & $\begin{array}{l}5273.6 \\
5562.2\end{array}$ & $\begin{array}{l}7914.0 \\
8398.0\end{array}$ \\
\hline $\begin{array}{r}2007 \text { Q2 } \\
\text { Q3 } \\
\text { Q4 }\end{array}$ & $\begin{array}{l}9.3 \\
9.2 \\
8.7\end{array}$ & $\begin{array}{l}1957.1 \\
1597.6 \\
1533.5\end{array}$ & $\begin{array}{ll}2 & 079.2 \\
2 & 177.7 \\
2 & 330.3\end{array}$ & $\begin{array}{l}2.8 \\
3.5 \\
3.8\end{array}$ & $\begin{array}{l}4048.4 \\
3788.0 \\
3876.2\end{array}$ & $\begin{array}{l}11.8 \\
10.2 \\
10.3\end{array}$ & $\begin{array}{l}- \\
- \\
-\end{array}$ & $\begin{array}{l}148.8 \\
156.7 \\
148.1\end{array}$ & $\begin{array}{r}10.3 \\
12.1 \\
9.4\end{array}$ & $\begin{array}{l}67.0 \\
62.9 \\
64.5\end{array}$ & $\begin{array}{l}288.0 \\
306.6 \\
319.1\end{array}$ & $\begin{array}{l}0.4 \\
0.4 \\
0.3\end{array}$ & $\begin{array}{l}105.3 \\
113.1 \\
115.9\end{array}$ & $\begin{array}{l}462.6 \\
455.5 \\
463.9\end{array}$ & $\begin{array}{l}825.3 \\
829.1 \\
866.6\end{array}$ & 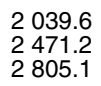 & $\begin{array}{l}3959.1 \\
4417.7 \\
4803.2\end{array}$ \\
\hline $\begin{array}{r}2008 \text { Q1 } \\
\text { Q2 } \\
\text { Q3 } \\
\text { Q4 }\end{array}$ & $\begin{array}{l}7.0 \\
6.4 \\
6.7 \\
8.1\end{array}$ & $\begin{array}{l}1709.4 \\
1574.9 \\
1771.7 \\
1888.6\end{array}$ & $\begin{array}{l}2584.1 \\
2279.1 \\
2409.5 \\
2527.5\end{array}$ & $\begin{array}{r}5.1 \\
11.8 \\
7.6 \\
11.5\end{array}$ & $\begin{array}{l}4305.6 \\
3572.3 \\
4195.4 \\
4 \quad 435.7\end{array}$ & $\begin{array}{l}8.9 \\
7.7 \\
9.3 \\
9.8\end{array}$ & $\begin{array}{l}- \\
- \\
- \\
-\end{array}$ & $\begin{array}{l}148.4 \\
141.6 \\
136.1 \\
135.9\end{array}$ & $\begin{array}{l}7.1 \\
7.4 \\
8.6 \\
5.1\end{array}$ & $\begin{array}{l}63.1 \\
67.4 \\
69.3 \\
65.5\end{array}$ & $\begin{array}{l}329.1 \\
322.9 \\
322.5 \\
401.5\end{array}$ & $\begin{array}{l}0.3 \\
0.3 \\
0.3 \\
0.2\end{array}$ & $\begin{array}{l}115.8 \\
119.4 \\
121.0 \\
153.8\end{array}$ & $\begin{array}{l}450.1 \\
434.3 \\
449.3 \\
446.1\end{array}$ & $\begin{array}{r}879.6 \\
859.8 \\
901.6 \\
1026.9\end{array}$ & $\begin{array}{l}4530.6 \\
4301.3 \\
4639.2 \\
9566.2\end{array}$ & $\begin{array}{r}6533.0 \\
6262.3 \\
6657.3 \\
11811.1\end{array}$ \\
\hline $\begin{array}{r}2009 \text { Q1 } \\
\text { Q2 } \\
\text { Q3 } \\
\text { Q4 }\end{array}$ & $\begin{array}{r}6.5 \\
7.5 \\
6.7 \\
12.8\end{array}$ & $\begin{array}{ll}1 & 851.7 \\
1 & 845.0 \\
1 & 939.7 \\
2 & 141.5\end{array}$ & $\begin{array}{ll}2 & 401.3 \\
2 & 169.2 \\
2 & 222.1 \\
2 & 187.3\end{array}$ & $\begin{array}{l}24.3 \\
14.5 \\
13.0 \\
16.9\end{array}$ & $\begin{array}{l}4283.8 \\
4036.2 \\
4181.4 \\
4358.5\end{array}$ & $\begin{array}{l}18.2 \\
22.9 \\
30.3 \\
37.6\end{array}$ & $\begin{array}{l}- \\
- \\
- \\
-\end{array}$ & $\begin{array}{r}123.0 \\
107.8 \\
103.2 \\
73.5\end{array}$ & $\begin{array}{l}4.4 \\
4.8 \\
5.7 \\
4.3\end{array}$ & $\begin{array}{l}66.2 \\
66.7 \\
81.7 \\
75.9\end{array}$ & $\begin{array}{l}412.5 \\
467.8 \\
539.9 \\
560.6\end{array}$ & $\begin{array}{l}0.4 \\
0.5 \\
0.6 \\
0.5\end{array}$ & $\begin{array}{l}169.5 \\
170.4 \\
177.4 \\
176.4\end{array}$ & $\begin{array}{l}399.2 \\
449.2 \\
536.5 \\
582.9\end{array}$ & $\begin{array}{ll}1 & 083.6 \\
1 & 036.7 \\
1 & 145.0 \\
1 & 128.6\end{array}$ & $\begin{array}{ll}9 & 556.3 \\
6 & 236.6 \\
6 & 132.1 \\
5 & 273.6\end{array}$ & $\begin{array}{r}11833.1 \\
8563.4 \\
8752.7 \\
7914.0\end{array}$ \\
\hline $\begin{array}{r}2010 \text { Q1 } \\
\text { Q2 } \\
\text { Q3 } \\
\text { Q4 }\end{array}$ & $\begin{array}{r}10.5 \\
8.9 \\
9.2 \\
10.4\end{array}$ & $\begin{array}{ll}2 & 281.3 \\
2 & 032.5^{\dagger} \\
2 & 004.6 \\
2 & 013.5\end{array}$ & $\begin{array}{l}2384.9^{\dagger} \\
22428.9 \\
2477.0 \\
2365.1\end{array}$ & $\begin{array}{r}14.6 \\
10.3 \\
7.8 \\
10.6\end{array}$ & $\begin{array}{l}4691.3^{\dagger} \\
4480.6 \\
4498.6 \\
4399.6\end{array}$ & $\begin{array}{l}33.1 \\
32.3 \\
30.9 \\
26.5\end{array}$ & $\begin{array}{l}- \\
- \\
- \\
-\end{array}$ & $\begin{array}{l}70.4^{\dagger} \\
57.4^{\dagger} \\
60.3 \\
56.2\end{array}$ & $\begin{array}{l}4.3^{\dagger} \\
4.3^{\dagger} \\
3.6 \\
3.0\end{array}$ & $\begin{array}{l}70.7^{\dagger} \\
63.8 \\
82.9 \\
76.8\end{array}$ & $\begin{array}{l}590.6^{\dagger} \\
629.4 \\
668.8 \\
671.1\end{array}$ & $\begin{array}{l}0.5 \\
0.5 \\
0.5 \\
0.6\end{array}$ & $\begin{array}{l}173.5 \\
163.9 \\
166.2 \\
176.1\end{array}$ & $\begin{array}{l}610.9^{\dagger} \\
546.5 \\
552.7 \\
583.2\end{array}$ & $\begin{array}{ll}1 & 236.5^{\dagger} \\
1 & 200.7 \\
1 & 236.1 \\
1 & 242.4\end{array}$ & 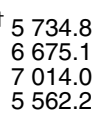 & $\begin{array}{l}8525.4^{\dagger} \\
9373.9 \\
9816.1 \\
8398.0\end{array}$ \\
\hline
\end{tabular}

Financial assets - continued

$\begin{array}{lr}\text { Loans } & \text { technical } \\ \text { Shares and other equity } & \text { reserves }\end{array}$

\begin{tabular}{|c|c|c|c|c|c|c|c|c|c|c|c|c|c|c|}
\hline & & & & & & & & \multirow{3}{*}{$\begin{array}{r}\text { Prepayme- } \\
\text { nts of } \\
\text { insurance } \\
\text { premiums } \\
\text { etc. }\end{array}$} & \multirow{3}{*}{$\begin{array}{r}\text { Other } \\
\text { accounts } \\
\text { receivab- } \\
\text { le/ } \\
\text { payable }\end{array}$} & \multirow[b]{3}{*}{$\begin{array}{r}\text { Total } \\
\text { financial } \\
\text { assets }\end{array}$} \\
\hline & $\begin{array}{c}\text { Short } \\
\text { term } \\
\text { loans }\end{array}$ & \multicolumn{4}{|c|}{ Long term loans } & \multirow[b]{2}{*}{ Total } & \multicolumn{3}{|c|}{ Excl mutual funds' shares } & \multirow[b]{2}{*}{$\begin{array}{r}\text { UK mutual } \\
\text { funds } \\
\text { shares }\end{array}$} & \multirow[b]{2}{*}{ Total } & & & \\
\hline & $\begin{array}{l}\text { by UK } \\
\text { MFl's }^{3}\end{array}$ & $\begin{array}{r}\text { Direct } \\
\text { investme- } \\
\text { nt }\end{array}$ & $\begin{array}{r}\text { Secured } \\
\text { on } \\
\text { dwellings }\end{array}$ & $\begin{array}{l}\text { Finance } \\
\text { leasing }\end{array}$ & Other & & $\begin{array}{r}\text { Quoted UK } \\
\text { shares }\end{array}$ & $\begin{array}{l}\text { Unquoted } \\
\text { UK shares }\end{array}$ & $\begin{array}{l}\text { By the } \\
\text { rest of } \\
\text { the world }\end{array}$ & & & & & \\
\hline & F411 & $\mathrm{F} 421$ & F422 & F423 & F424 & $\mathrm{F} 4$ & F514 & F515 & F519 & F521 & F5 & F62 & $\mathrm{F} 7$ & FA \\
\hline $\begin{array}{l}2004 \\
2005 \\
2006 \\
2007 \\
2008\end{array}$ & $\begin{array}{r}\text { NLKQ } \\
1487.7 \\
1754.5 \\
1969.0 \\
2537.7 \\
2990.4\end{array}$ & $\begin{array}{r}\text { NLKW } \\
25.3 \\
28.9 \\
22.7 \\
23.2 \\
27.8\end{array}$ & $\begin{array}{r}\text { NLKZ } \\
880.0 \\
964.1 \\
1075.5 \\
1179.4 \\
1222.4\end{array}$ & $\begin{array}{r}\text { NLLD } \\
25.9 \\
26.4 \\
27.1 \\
27.7 \\
28.2\end{array}$ & $\begin{array}{r}\text { NLLE } \\
91.9 \\
91.8 \\
115.8 \\
128.2 \\
109.8\end{array}$ & $\begin{array}{r}\text { NLKO } \\
2510.8 \\
2865.7 \\
3210.1 \\
3896.2 \\
4378.6\end{array}$ & $\begin{array}{l}\text { NLLL } \\
678.0 \\
752.6 \\
805.9 \\
779.2 \\
432.6\end{array}$ & $\begin{array}{l}\text { NLLM } \\
263.9 \\
280.6 \\
332.1 \\
313.7 \\
336.0\end{array}$ & $\begin{array}{l}\text { NLLQ } \\
526.5 \\
682.2 \\
771.0 \\
878.5 \\
718.4\end{array}$ & $\begin{array}{l}\text { NLLU } \\
164.1 \\
243.3 \\
286.0 \\
342.1 \\
303.9\end{array}$ & $\begin{array}{rr} & \text { NLLG } \\
1 & 632.5 \\
1 & 958.6 \\
2 & 195.1 \\
2 & 313.6 \\
1 & 790.9\end{array}$ & $\begin{array}{r}\text { NLLZ } \\
1.6 \\
1.9 \\
1.8 \\
1.4 \\
1.4\end{array}$ & $\begin{array}{r}\text { NLMA } \\
62.4 \\
63.8 \\
99.2 \\
91.4 \\
69.9\end{array}$ & $\begin{array}{r}\text { NLIZ } \\
8208.2 \\
9752.0 \\
12743.3 \\
14981.8 \\
22487.6\end{array}$ \\
\hline $\begin{array}{l}2009 \\
2010\end{array}$ & $\begin{array}{l}2504.8 \\
2475.1\end{array}$ & $\begin{array}{l}40.4 \\
41.8\end{array}$ & $\begin{array}{l}1230.9 \\
1234.1\end{array}$ & $\begin{array}{l}28.7 \\
29.2\end{array}$ & $\begin{array}{l}130.5 \\
164.7\end{array}$ & $\begin{array}{l}3935.2 \\
3944.9\end{array}$ & $\begin{array}{l}589.3 \\
650.3\end{array}$ & $\begin{array}{l}326.1 \\
362.3\end{array}$ & $\begin{array}{l}841.8 \\
933.7\end{array}$ & $\begin{array}{l}402.6 \\
476.5\end{array}$ & $\begin{array}{l}2159.7 \\
2422.8\end{array}$ & $\begin{array}{l}1.3 \\
1.4\end{array}$ & $\begin{array}{l}68.2 \\
69.9\end{array}$ & $\begin{array}{l}18436.9 \\
19236.5\end{array}$ \\
\hline $\begin{array}{r}2007 \text { Q2 } \\
\text { Q3 } \\
\text { Q4 }\end{array}$ & $\begin{array}{l}2279.0 \\
2443.0 \\
2537.7\end{array}$ & $\begin{array}{l}23.2 \\
22.9 \\
23.2\end{array}$ & $\begin{array}{ll}1 & 127.0 \\
1 & 158.1 \\
1 & 179.4\end{array}$ & $\begin{array}{l}27.4 \\
27.6 \\
27.7\end{array}$ & $\begin{array}{l}122.9 \\
129.8 \\
128.2\end{array}$ & $\begin{array}{l}3579.5 \\
3781.4 \\
3896.2\end{array}$ & $\begin{array}{l}810.5 \\
801.2 \\
779.2\end{array}$ & $\begin{array}{l}309.8 \\
305.8 \\
313.7\end{array}$ & $\begin{array}{l}832.9 \\
850.8 \\
878.5\end{array}$ & $\begin{array}{l}326.5 \\
330.9 \\
342.1\end{array}$ & $\begin{array}{l}2279.7 \\
2288.7 \\
2313.6\end{array}$ & $\begin{array}{l}1.5 \\
1.4 \\
1.4\end{array}$ & $\begin{array}{r}100.8 \\
96.0 \\
91.4\end{array}$ & $\begin{array}{l}13969.0 \\
14373.1 \\
14981.8\end{array}$ \\
\hline $\begin{array}{r}2008 \text { Q1 } \\
\text { Q2 } \\
\text { Q3 } \\
\text { Q4 }\end{array}$ & $\begin{array}{l}2753.2 \\
2621.9 \\
2792.8 \\
2990.4\end{array}$ & $\begin{array}{l}30.2 \\
29.8 \\
29.4 \\
27.8\end{array}$ & $\begin{array}{ll}1 & 195.6 \\
1 & 209.0 \\
1 & 219.1 \\
1 & 222.4\end{array}$ & $\begin{array}{l}27.8 \\
28.0 \\
28.1 \\
28.2\end{array}$ & $\begin{array}{l}126.5 \\
119.8 \\
114.6 \\
109.8\end{array}$ & $\begin{array}{l}4133.3 \\
4008.5 \\
4184.0 \\
4378.6\end{array}$ & $\begin{array}{l}688.2 \\
628.5 \\
537.3 \\
432.6\end{array}$ & $\begin{array}{l}318.4 \\
325.1 \\
328.4 \\
336.0\end{array}$ & $\begin{array}{l}814.0 \\
790.1 \\
758.5 \\
718.4\end{array}$ & $\begin{array}{l}322.6 \\
330.1 \\
305.9 \\
303.9\end{array}$ & $\begin{array}{ll}2 & 143.1 \\
2 & 073.7 \\
1 & 930.0 \\
1 & 790.9\end{array}$ & $\begin{array}{l}1.4 \\
1.4 \\
1.4 \\
1.4\end{array}$ & $\begin{array}{l}85.1 \\
78.5 \\
73.6 \\
69.9\end{array}$ & $\begin{array}{ll}17 & 201.5 \\
16 & 296.6 \\
17 & 041.7 \\
22 & 487.6\end{array}$ \\
\hline $\begin{array}{r}2009 \text { Q1 } \\
\text { Q2 } \\
\text { Q3 } \\
\text { Q4 }\end{array}$ & $\begin{array}{l}2895.2 \\
2573.1 \\
2566.5 \\
2504.8\end{array}$ & $\begin{array}{l}37.3 \\
38.0 \\
39.4 \\
40.4\end{array}$ & $\begin{array}{ll}1 & 221.8 \\
1 & 222.9 \\
1 & 227.3 \\
1 & 230.9\end{array}$ & $\begin{array}{l}28.3 \\
28.5 \\
28.6 \\
28.7\end{array}$ & $\begin{array}{l}124.7 \\
133.4 \\
154.1 \\
130.5\end{array}$ & $\begin{array}{l}4307.3 \\
3995.8 \\
4016.0 \\
3935.2\end{array}$ & $\begin{array}{l}385.7 \\
449.2 \\
566.3 \\
589.3\end{array}$ & $\begin{array}{l}335.1 \\
322.0 \\
322.8 \\
326.1\end{array}$ & $\begin{array}{l}669.3 \\
712.5 \\
807.2 \\
841.8\end{array}$ & $\begin{array}{l}285.3 \\
314.9 \\
385.8 \\
402.6\end{array}$ & $\begin{array}{ll}1 & 675.4 \\
1 & 798.7 \\
2 & 082.0 \\
2 & 159.7\end{array}$ & $\begin{array}{l}1.4 \\
1.3 \\
1.4 \\
1.3\end{array}$ & $\begin{array}{l}68.8 \\
67.9 \\
67.8 \\
68.2\end{array}$ & $\begin{array}{ll}22 & 169.7 \\
18 & 463.4 \\
19 & 101.2 \\
18 & 436.9\end{array}$ \\
\hline $\begin{array}{r}2010 \text { Q1 } \\
\text { Q2 } \\
\text { Q3 } \\
\text { Q4 }\end{array}$ & $\begin{array}{ll}2 & 594.2 \\
2 & 497.4 \\
2 & 530.8^{\dagger} \\
2 & 475.1\end{array}$ & $\begin{array}{l}40.1^{\dagger} \\
40.0 \\
41.4 \\
41.8\end{array}$ & $\begin{array}{ll}1 & 231.9 \\
1 & 233.0^{\dagger} \\
1 & 236.4^{\dagger} \\
1 & 234.1\end{array}$ & $\begin{array}{l}28.8 \\
28.9^{\dagger} \\
29.0^{\dagger} \\
29.2\end{array}$ & $\begin{array}{l}193.6^{\dagger} \\
162.8 \\
177.3 \\
164.7\end{array}$ & $\begin{array}{l}4088.7^{\dagger} \\
3962.1 \\
4015.0 \\
3944.9\end{array}$ & $\begin{array}{l}616.8^{\dagger} \\
544.8^{-16.4} \\
616.4 \\
650.3\end{array}$ & $\begin{array}{l}360.7^{\dagger} \\
359.6 \\
357.8 \\
362.3\end{array}$ & $\begin{array}{l}876.2^{\dagger} \\
792.9 \\
852.5 \\
933.7\end{array}$ & $\begin{array}{l}421.8^{\dagger} \\
374.5 \\
433.5 \\
476.5\end{array}$ & $\begin{array}{l}2275.4^{\dagger} \\
2071.8 \\
2260.2 \\
22422.8\end{array}$ & $\begin{array}{l}1.3 \\
1.4 \\
1.4 \\
1.4\end{array}$ & $\begin{array}{l}69.0 \\
69.3 \\
69.8 \\
69.9\end{array}$ & $\begin{array}{l}19651.1^{\dagger} \\
19959.1 \\
20661.0 \\
19236.5\end{array}$ \\
\hline $\begin{array}{l}1 \text { Mone } \\
2 \text { Mone } \\
3 \text { All loa } \\
\text { term }\end{array}$ & inanc & stitution & & & & & & & & & & & $\begin{array}{l}\text { Nationa } \\
\text { Bank }\end{array}$ & $\begin{array}{l}\text { Statistics; } \\
\text { f England }\end{array}$ \\
\hline
\end{tabular}




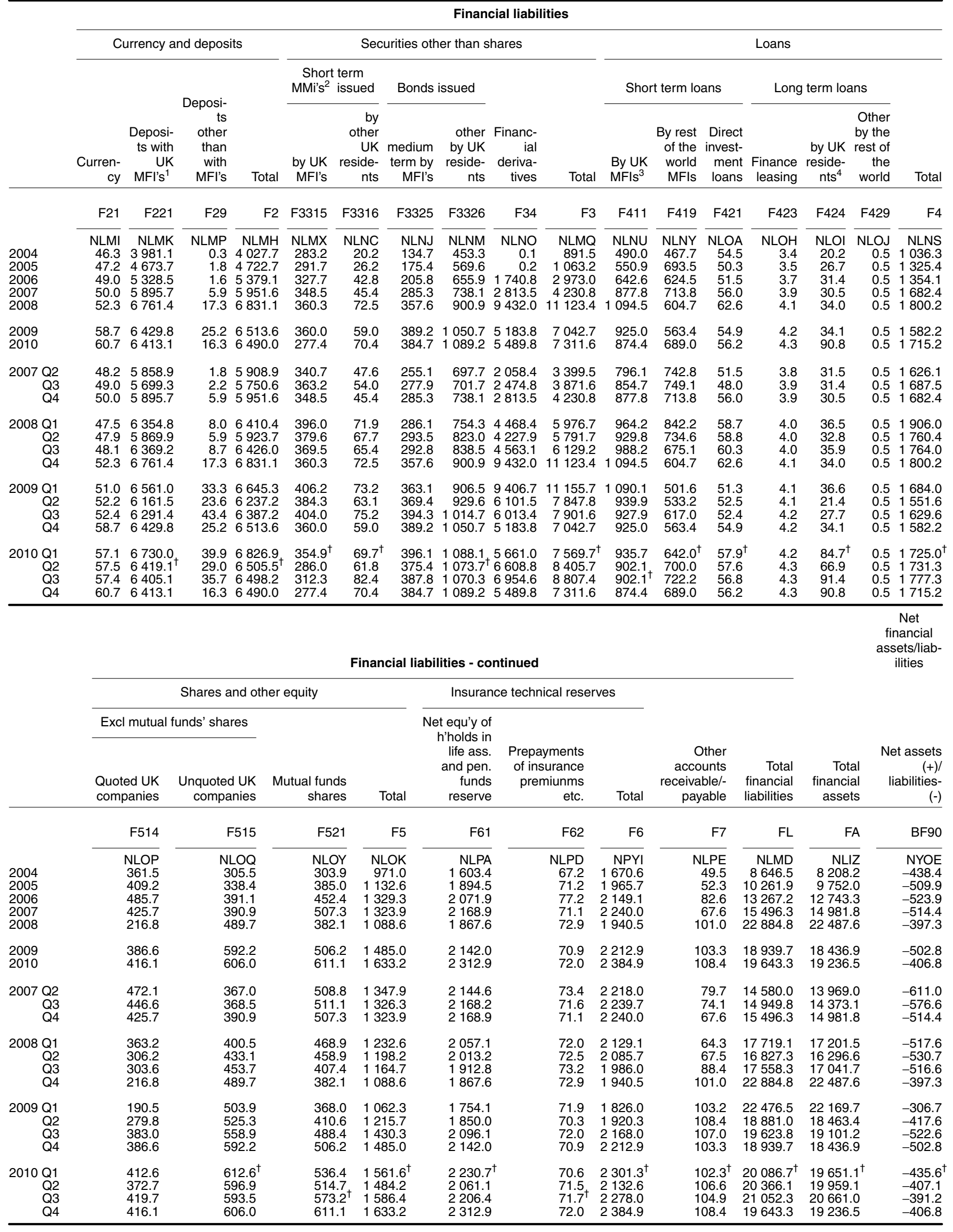

1 Monetary financial institutions

2 Money market instruments

3 All loans secured on dwellings and all finance leasing are treated as long Bank of England

term loans

4 Other than direct investment loans, loans secured on dwellings and loans

for finance leasing 


\begin{tabular}{|c|c|c|c|c|c|c|c|c|c|c|c|c|c|c|c|c|c|}
\hline & \multicolumn{17}{|c|}{ Financial assets(MFI) } \\
\hline & \multicolumn{5}{|c|}{ Currency and deposits } & \multicolumn{12}{|c|}{ Securities other than shares } \\
\hline & \multirow[b]{2}{*}{$\begin{array}{r}\text { Curren- } \\
\text { cy }\end{array}$} & \multirow[b]{2}{*}{$\begin{array}{r}\text { Deposi- } \\
\text { ts with } \\
\text { UK } \\
\text { MFI's }^{1}\end{array}$} & \multirow[b]{2}{*}{$\begin{array}{r}\text { Deposi- } \\
\text { ts with } \\
\text { rest of } \\
\text { the } \\
\text { world } \\
\text { MFI's }\end{array}$} & \multirow[b]{2}{*}{$\begin{array}{r}\text { Deposi- } \\
\text { ts } \\
\text { other } \\
\text { than } \\
\text { with } \\
\text { MFl's }\end{array}$} & \multirow[b]{2}{*}{ Total } & \multicolumn{5}{|c|}{ Short term MMl's ${ }^{2}$ issued } & \multicolumn{6}{|c|}{ Bonds issued } & \multirow[b]{2}{*}{ Total } \\
\hline & & & & & & $\begin{array}{r}\text { by UK } \\
\text { general } \\
\text { govern- } \\
\text { ment }\end{array}$ & $\begin{array}{r}\text { UK } \\
\text { local } \\
\text { author- } \\
\text { ity } \\
\text { bills }\end{array}$ & $\begin{array}{l} \\
\text { by UK } \\
\text { MFl's }\end{array}$ & $\begin{array}{r}\text { by } \\
\text { other } \\
\text { UK } \\
\text { reside- } \\
\text { nts }\end{array}$ & $\begin{array}{r}\text { by the } \\
\text { rest of } \\
\text { the } \\
\text { world }\end{array}$ & $\begin{array}{r}\text { by UK } \\
\text { central } \\
\text { govern- } \\
\text { ment }\end{array}$ & $\begin{array}{r}\text { by UK } \\
\text { local } \\
\text { author- } \\
\text { ities }\end{array}$ & $\begin{array}{r}\text { Medium } \\
\text { term by } \\
\text { MFl's }\end{array}$ & $\begin{array}{r}\text { Other } \\
\text { by UK } \\
\text { reside- } \\
\text { nts }\end{array}$ & $\begin{array}{l}\text { by the } \\
\text { rest of } \\
\text { the } \\
\text { world }\end{array}$ & $\begin{array}{r}\text { Financ- } \\
\text { ial } \\
\text { deriva- } \\
\text { tives }\end{array}$ & \\
\hline & $\mathrm{F} 21$ & $\mathrm{~F} 221$ & F229 & F29 & $\mathrm{F} 2$ & F3311 & F3312 & F3315 & F3316 & F3319 & F3321 & F3322 & F3325 & F3326 & F3329 & F34 & F3 \\
\hline $\begin{array}{l}2004 \\
2005 \\
2006 \\
2007 \\
2008\end{array}$ & $\begin{array}{r}\text { NNSY } \\
11.3 \\
10.2 \\
10.0 \\
8.6 \\
8.0\end{array}$ & $\begin{array}{r}\text { NNTA } \\
831.9 \\
1000.5 \\
1282.2 \\
842.7 \\
924.6\end{array}$ & $\begin{array}{rr} & \text { NNTE } \\
1 & 006.9 \\
1 & 199.7 \\
1 & 286.0 \\
1 & 714.1 \\
2 & 092.6\end{array}$ & $\begin{array}{r}\text { NNTF } \\
- \\
- \\
- \\
- \\
-\end{array}$ & $\begin{array}{r}\text { NNSX } \\
-\quad 1850.1 \\
-2210.4 \\
-2578.1 \\
-\quad 2565.5 \\
-\quad 3025.3\end{array}$ & $\begin{array}{r}\text { NNTI } \\
16.1 \\
15.8 \\
12.1 \\
5.6 \\
3.1\end{array}$ & $\begin{array}{r}\text { NNTM } \\
- \\
- \\
- \\
- \\
-\end{array}$ & $\begin{array}{r}\text { NNTN } \\
90.9 \\
96.1 \\
98.4 \\
88.7 \\
72.5\end{array}$ & $\begin{array}{r}\text { NNTS } \\
1.9 \\
2.9 \\
5.0 \\
1.3 \\
0.5\end{array}$ & $\begin{array}{r}\text { NNTT } \\
37.0 \\
39.6 \\
42.8 \\
53.8 \\
57.1\end{array}$ & $\begin{array}{r}\text { NNTV } \\
-1.4 \\
-2.8 \\
-7.7 \\
-5.5 \\
25.7\end{array}$ & $\begin{array}{r}\text { NNTY } \\
- \\
- \\
- \\
- \\
-\end{array}$ & $\begin{array}{r}\text { NNTZ } \\
23.4 \\
25.2 \\
24.5 \\
25.2 \\
43.0\end{array}$ & $\begin{array}{r}\text { NNUC } \\
90.0 \\
133.6 \\
189.4 \\
209.4 \\
230.5\end{array}$ & $\begin{array}{r}\text { NNUD } \\
359.7 \\
413.7 \\
479.1 \\
555.6 \\
568.2\end{array}$ & $\begin{array}{r}\text { NNUE } \\
- \\
\\
11481 . \overline{4} \\
2368.4 \\
7634.7\end{array}$ & $\begin{array}{r}\text { NNTG } \\
617.7 \\
724.1 \\
2325.0 \\
3302.5 \\
8635.3\end{array}$ \\
\hline $\begin{array}{l}2009 \\
2010\end{array}$ & $\begin{array}{l}12.7 \\
10.3\end{array}$ & $\begin{array}{r}1142.2 \\
938.3\end{array}$ & $\begin{array}{l}1779.3 \\
1876.3\end{array}$ & $\begin{array}{l}- \\
-\end{array}$ & $\begin{array}{r}2934.2 \\
-2824.8\end{array}$ & $\begin{array}{l}22.8 \\
10.9\end{array}$ & $\begin{array}{l}- \\
-\end{array}$ & $\begin{array}{l}32.0 \\
21.0\end{array}$ & $\begin{array}{l}- \\
-\end{array}$ & $\begin{array}{l}65.4 \\
65.0\end{array}$ & $\begin{array}{l}228.8 \\
290.2\end{array}$ & $\begin{array}{l}- \\
-\end{array}$ & $\begin{array}{l}54.3 \\
57.2\end{array}$ & $\begin{array}{l}318.8 \\
357.7\end{array}$ & $\begin{array}{l}504.0 \\
480.0\end{array}$ & $\begin{array}{l}4104.9 \\
4261.7\end{array}$ & $\begin{array}{l}5331.0 \\
5543.7\end{array}$ \\
\hline $\begin{array}{r}2007 \text { Q2 } \\
\text { Q3 } \\
\text { Q4 }\end{array}$ & $\begin{array}{l}9.3 \\
9.2 \\
8.6\end{array}$ & $\begin{array}{r}1318.1 \\
937.8 \\
842.7\end{array}$ & $\begin{array}{l}1483.7 \\
1578.9 \\
1714.1\end{array}$ & & $\begin{array}{r}2811.0 \\
-2525.9 \\
-2565.5\end{array}$ & $\begin{array}{l}8.0 \\
4.7 \\
5.6\end{array}$ & $\begin{array}{l}- \\
- \\
-\end{array}$ & $\begin{array}{l}95.1 \\
95.1 \\
88.7\end{array}$ & $\begin{array}{l}2.9 \\
2.5 \\
1.3\end{array}$ & $\begin{array}{l}51.8 \\
50.4 \\
53.8\end{array}$ & $\begin{array}{r}-10.1 \\
-8.1 \\
-5.5\end{array}$ & $\begin{array}{l}- \\
- \\
-\end{array}$ & $\begin{array}{l}27.0 \\
24.0 \\
25.2\end{array}$ & $\begin{array}{l}203.5 \\
206.4 \\
209.4\end{array}$ & $\begin{array}{l}531.5 \\
537.4 \\
555.6\end{array}$ & $\begin{array}{l}1705.2 \\
2139.3 \\
2368.4\end{array}$ & $\begin{array}{l}2614.9 \\
3051.6 \\
3302.5\end{array}$ \\
\hline $\begin{array}{r}2008 \text { Q1 } \\
\text { Q2 } \\
\text { Q3 } \\
\text { Q4 }\end{array}$ & $\begin{array}{l}6.9 \\
6.3 \\
6.6 \\
8.0\end{array}$ & $\begin{array}{l}930.5 \\
802.8 \\
946.9 \\
924.6\end{array}$ & $\begin{array}{ll}1 & 914.1 \\
1 & 722.8 \\
1 & 868.0 \\
2 & 092.6\end{array}$ & & $\begin{array}{r}2851.5 \\
-2532.0 \\
-2821.6 \\
-3025.3\end{array}$ & $\begin{array}{l}4.4 \\
4.4 \\
2.0 \\
3.1\end{array}$ & $\begin{array}{l}- \\
- \\
- \\
-\end{array}$ & $\begin{array}{l}90.0 \\
79.8 \\
77.5 \\
72.5\end{array}$ & $\begin{array}{l}0.9 \\
0.9 \\
0.8 \\
0.5\end{array}$ & $\begin{array}{l}52.1 \\
56.9 \\
60.5 \\
57.1\end{array}$ & $\begin{array}{r}-2.8 \\
-2.9 \\
3.2 \\
25.7\end{array}$ & $\begin{array}{l}- \\
- \\
- \\
-\end{array}$ & $\begin{array}{l}25.1 \\
25.8 \\
26.7 \\
43.0\end{array}$ & $\begin{array}{l}218.7 \\
209.8 \\
228.2 \\
230.5\end{array}$ & $\begin{array}{l}555.5 \\
517.5 \\
529.8 \\
568.2\end{array}$ & $\begin{array}{l}3666.2 \\
3661.6 \\
35950.6 \\
7634.7\end{array}$ & $\begin{array}{l}4810.1 \\
4553.8 \\
4879.4 \\
8635.3\end{array}$ \\
\hline $\begin{array}{r}2009 \text { Q1 } \\
\text { Q2 } \\
\text { Q3 } \\
\text { Q4 }\end{array}$ & $\begin{array}{r}6.4 \\
7.4 \\
6.6 \\
12.7\end{array}$ & $\begin{array}{r}846.8 \\
884.3 \\
948.1 \\
1142.2\end{array}$ & $\begin{array}{ll}2 & 039.4 \\
1 & 814.7 \\
1 & 819.2 \\
1 & 779.3\end{array}$ & & $\begin{array}{r}2892.7 \\
-2706.4 \\
-2773.9 \\
-2934.2\end{array}$ & $\begin{array}{r}5.8 \\
8.8 \\
13.9 \\
22.8\end{array}$ & $\begin{array}{l}- \\
- \\
- \\
-\end{array}$ & $\begin{array}{l}58.6 \\
50.2 \\
51.3 \\
32.0\end{array}$ & $\begin{array}{r}0.3 \\
0.2 \\
0.1 \\
-\end{array}$ & $\begin{array}{l}57.9 \\
57.6 \\
70.2 \\
65.4\end{array}$ & $\begin{array}{r}56.6 \\
133.3 \\
197.9 \\
228.8\end{array}$ & $\begin{array}{l}- \\
- \\
- \\
-\end{array}$ & $\begin{array}{l}58.3 \\
57.2 \\
56.3 \\
54.3\end{array}$ & $\begin{array}{l}187.2 \\
219.5 \\
294.2 \\
318.8\end{array}$ & $\begin{array}{l}573.1 \\
497.3 \\
526.2 \\
504.0\end{array}$ & $\begin{array}{l}7483.7 \\
4897.1 \\
4835.3 \\
4104.9\end{array}$ & $\begin{array}{ll}8 & 481.5 \\
5 & 921.2 \\
6 & 045.3 \\
5 & 331.0\end{array}$ \\
\hline $\begin{array}{r}2010 \text { Q1 } \\
\text { Q2 } \\
\text { Q3 } \\
\text { Q4 }\end{array}$ & $\begin{array}{r}10.4 \\
8.8 \\
9.1 \\
10.3\end{array}$ & $\begin{array}{r}1109.1 \\
893.8 \\
888.6 \\
938.3\end{array}$ & $\begin{array}{l}1897.0 \\
1933.4 \\
1956.2^{\dagger} \\
1876.3\end{array}$ & & $\begin{array}{l}3016.5 \\
2836.1 \\
-2853.9^{\dagger} \\
-2824.8\end{array}$ & $\begin{array}{l}15.6 \\
15.4 \\
13.8 \\
10.9\end{array}$ & $\begin{array}{l}- \\
- \\
- \\
-\end{array}$ & $\begin{array}{l}30.1 \\
21.2 \\
20.8 \\
21.0\end{array}$ & $\begin{array}{l}0.1 \\
0.3 \\
0.2 \\
-\end{array}$ & $\begin{array}{l}58.3 \\
50.4 \\
68.0 \\
65.0\end{array}$ & $\begin{array}{l}251.6 \\
276.5 \\
287.1 \\
290.2\end{array}$ & $\begin{array}{l}- \\
- \\
- \\
-\end{array}$ & $\begin{array}{l}46.6 \\
43.1 \\
40.9 \\
57.2\end{array}$ & $\begin{array}{l}366.1^{\dagger} \\
307.9 \\
337.6 \\
357.7\end{array}$ & $\begin{array}{l}535.9 \\
493.8^{\dagger} \\
509.5 \\
480.0\end{array}$ & $\begin{array}{r}4464.6 \\
5116.8 \\
5373.9 \\
4261.7\end{array}$ & $\begin{array}{l}5768.9^{\dagger} \\
6325.5 \\
6651.8 \\
5543.7\end{array}$ \\
\hline
\end{tabular}

Financial assets - continued

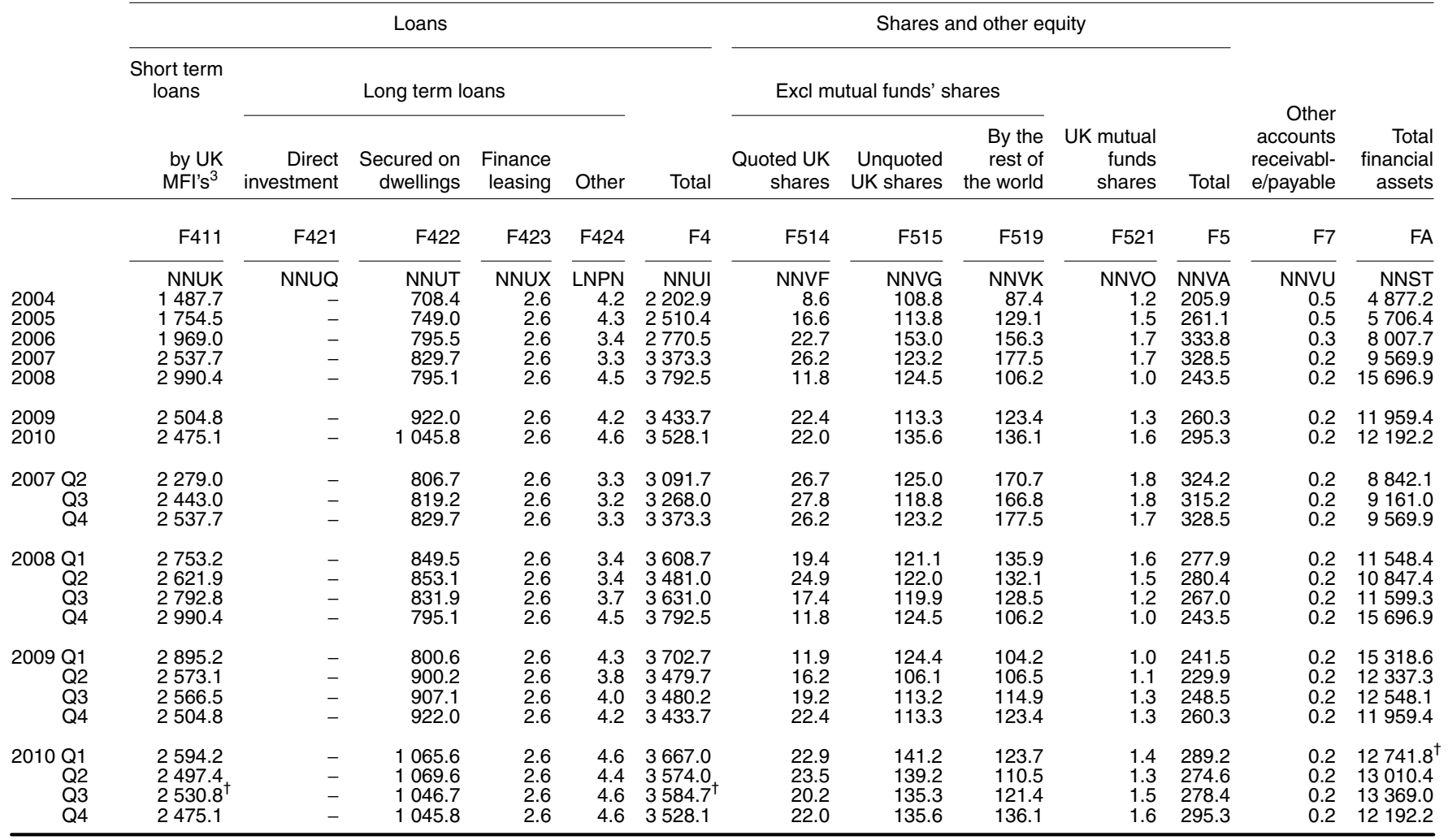

1 Monetary financial institutions

2 Money market instruments

3 All loans secured on dwellings and all finance leasing are treated as long Bank of England

term loans

4 Other than direct investment loans, loans secured on dwellings and loans

for finance leasing 


\begin{tabular}{|c|c|c|c|c|c|c|c|c|c|c|c|c|c|}
\hline & \multicolumn{13}{|c|}{ Financial liabilities (MFI) } \\
\hline & \multicolumn{3}{|c|}{ Currency and deposits } & \multicolumn{5}{|c|}{ Securities other than shares } & \multicolumn{5}{|c|}{ Loans } \\
\hline & \multirow[b]{2}{*}{ Currency } & \multirow[b]{2}{*}{$\begin{array}{c}\text { Deposits } \\
\text { with UK } \\
\text { MFIs }^{1}\end{array}$} & \multirow[b]{2}{*}{ Total } & \multirow{2}{*}{$\begin{array}{l}\text { Short term } \\
\text { MMl's }^{2} \\
\text { issued }\end{array}$} & \multicolumn{2}{|c|}{ Bonds issued } & \multirow[b]{2}{*}{$\begin{array}{r}\text { Financial } \\
\text { derivativ- } \\
\text { es }\end{array}$} & \multirow[b]{2}{*}{ Total } & \multirow{2}{*}{$\begin{array}{l}\text { Short term } \\
\text { loans }\end{array}$} & \multicolumn{3}{|c|}{ Long term loans } & \multirow[b]{2}{*}{ Total } \\
\hline & & & & & $\begin{array}{r}\text { medium } \\
\text { term by UK } \\
\text { MFIs }\end{array}$ & $\begin{array}{r}\text { other by } \\
\text { UK } \\
\text { residents }\end{array}$ & & & & $\begin{array}{r}\text { Direct } \\
\text { investment }\end{array}$ & $\begin{array}{r}\text { Finance } \\
\text { leasing }\end{array}$ & $\begin{array}{r}\text { by UK } \\
\text { residents }{ }^{4}\end{array}$ & \\
\hline & F21 & F221 & $\mathrm{F} 2$ & F3315 & F3325 & F3326 & F34 & F3 & $\mathrm{F} 411$ & $\mathrm{~F} 421$ & F423 & $\mathrm{F} 424$ & $\mathrm{~F} 4$ \\
\hline $\begin{array}{l}2004 \\
2005 \\
2006 \\
2007 \\
2008\end{array}$ & $\begin{array}{r}\text { NNWC } \\
46.3 \\
47.2 \\
49.0 \\
50.0 \\
52.3\end{array}$ & $\begin{array}{l}\text { NNWE } \\
3981.1 \\
4673.7 \\
5328.5 \\
5895.7 \\
6761.4\end{array}$ & $\begin{array}{l}\text { NNWB } \\
4027.4 \\
4720.9 \\
5377.5 \\
5945.7 \\
6813.8\end{array}$ & $\begin{array}{r}\text { NNWR } \\
283.2 \\
291.7 \\
327.7 \\
348.5 \\
360.3\end{array}$ & $\begin{array}{l}\text { NNXD } \\
134.7 \\
175.4 \\
205.8 \\
285.3 \\
357.6\end{array}$ & $\begin{array}{r}\text { NNXG } \\
119.4 \\
138.8 \\
142.3 \\
108.5 \\
225.5\end{array}$ & $\begin{array}{rr} & \text { NNXI } \\
& 0.1 \\
& 0.2 \\
1 & 499.3 \\
2 & 357.1 \\
7 & 517.6\end{array}$ & $\begin{array}{r}\text { NNWK } \\
537.5 \\
606.3 \\
2175.1 \\
3099.4 \\
8461.0\end{array}$ & $\begin{array}{r}\text { NNXO } \\
- \\
- \\
- \\
- \\
-\end{array}$ & $\begin{array}{r}\text { NNXU } \\
1.3 \\
1.3 \\
1.3 \\
1.3 \\
1.2\end{array}$ & $\begin{array}{r}\text { NNYB } \\
1.8 \\
1.9 \\
2.0 \\
2.1 \\
2.2\end{array}$ & $\begin{array}{r}\text { NNYC } \\
- \\
- \\
- \\
- \\
-\end{array}$ & $\begin{array}{r}\text { NNXM } \\
3.1 \\
3.2 \\
3.3 \\
3.3 \\
3.3\end{array}$ \\
\hline $\begin{array}{r}2007 \text { Q2 } \\
\text { Q3 } \\
\text { Q4 }\end{array}$ & $\begin{array}{l}48.2 \\
49.0 \\
50.0\end{array}$ & $\begin{array}{l}5858.9 \\
5699.3 \\
5895.7\end{array}$ & $\begin{array}{l}5907.1 \\
5 \text { 748.3 } \\
5945.7\end{array}$ & $\begin{array}{l}340.7 \\
363.2 \\
348.5\end{array}$ & $\begin{array}{l}255.1 \\
277.9 \\
285.3\end{array}$ & $\begin{array}{l}116.6 \\
104.4 \\
108.5\end{array}$ & $\begin{array}{l}1703.8 \\
2120.7 \\
2357.1\end{array}$ & $\begin{array}{l}2416.1 \\
2866.2 \\
3099.4\end{array}$ & $\begin{array}{l}- \\
- \\
-\end{array}$ & $\begin{array}{l}1.3 \\
1.3 \\
1.3\end{array}$ & $\begin{array}{l}2.0 \\
2.1 \\
2.1\end{array}$ & $\begin{array}{l}- \\
- \\
-\end{array}$ & $\begin{array}{l}3.3 \\
3.3 \\
3.3\end{array}$ \\
\hline $\begin{array}{r}2008 \text { Q1 } \\
\text { Q2 } \\
\text { Q3 } \\
\text { Q4 }\end{array}$ & $\begin{array}{l}47.5 \\
47.9 \\
48.1 \\
52.3\end{array}$ & $\begin{array}{l}6354.8 \\
5869.9 \\
6369.2 \\
6761.4\end{array}$ & 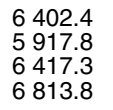 & $\begin{array}{l}396.0 \\
379.6 \\
369.5 \\
360.3\end{array}$ & $\begin{array}{l}286.1 \\
293.5 \\
292.8 \\
357.6\end{array}$ & $\begin{array}{l}130.6 \\
194.2 \\
221.5 \\
225.5\end{array}$ & $\begin{array}{l}3796.3 \\
3586.7 \\
3879.3 \\
7517.6\end{array}$ & $\begin{array}{l}4609.0 \\
4454.0 \\
4763.1 \\
8461.0\end{array}$ & $\begin{array}{l}- \\
- \\
- \\
-\end{array}$ & $\begin{array}{l}1.3 \\
1.3 \\
1.2 \\
1.2\end{array}$ & $\begin{array}{l}2.1 \\
2.1 \\
2.2 \\
2.2\end{array}$ & $\begin{array}{l}- \\
- \\
- \\
-\end{array}$ & $\begin{array}{l}3.4 \\
3.4 \\
3.3 \\
3.3\end{array}$ \\
\hline $\begin{array}{r}2009 \text { Q1 } \\
\text { Q2 } \\
\text { Q3 } \\
\text { Q4 }\end{array}$ & $\begin{array}{l}51.0 \\
52.2 \\
52.4 \\
58.7\end{array}$ & $\begin{array}{ll}6 & 561.0 \\
6 & 161.5 \\
6 & 291.4 \\
6 & 429.8\end{array}$ & $\begin{array}{l}6612.0 \\
6213.6 \\
6343.8 \\
6488.5\end{array}$ & $\begin{array}{l}406.2 \\
384.3 \\
404.0 \\
360.0\end{array}$ & $\begin{array}{l}363.1 \\
369.4 \\
394.3 \\
389.2\end{array}$ & $\begin{array}{l}260.4 \\
269.0 \\
260.5 \\
271.2\end{array}$ & $\begin{array}{l}7384.2 \\
4808.7 \\
4761.9 \\
4051.8\end{array}$ & 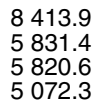 & $\begin{array}{l}- \\
- \\
- \\
-\end{array}$ & $\begin{array}{l}1.1 \\
1.1 \\
1.1 \\
1.0\end{array}$ & $\begin{array}{l}2.2 \\
2.2 \\
2.2 \\
2.3\end{array}$ & $\begin{array}{l}- \\
- \\
- \\
-\end{array}$ & $\begin{array}{l}3.3 \\
3.3 \\
3.3 \\
3.3\end{array}$ \\
\hline $\begin{array}{r}2010 \text { Q1 } \\
\text { Q2 } \\
\text { Q3 } \\
\text { Q4 }\end{array}$ & $\begin{array}{l}57.1 \\
57.5 \\
57.4 \\
60.7\end{array}$ & $\begin{array}{ll}6 & 730.0^{\dagger} \\
6 & 419.1^{\dagger} \\
6 & 405.1 \\
6 & 413.1\end{array}$ & $\begin{array}{ll}6 & 787.0^{\dagger} \\
6 & 476.6 \\
6 & 462.5 \\
6 & 473.7\end{array}$ & $\begin{array}{l}354.9^{\dagger} \\
286.0 \\
312.3 \\
277.4\end{array}$ & $\begin{array}{l}396.1 \\
375.4 \\
387.8 \\
384.7\end{array}$ & $\begin{array}{l}289.7^{\dagger} \\
322.1 \\
331.4 \\
336.7\end{array}$ & $\begin{array}{ll}4 & 425.0 \\
5 & 076.8 \\
5 & 346.7 \\
4 & 224.6\end{array}$ & $\begin{array}{ll}5 & 465.7^{\dagger} \\
6 & 060.3 \\
6 & 378.2 \\
5 & 223.3\end{array}$ & $\begin{array}{l}- \\
- \\
- \\
-\end{array}$ & $\begin{array}{l}1.0 \\
1.0 \\
1.0 \\
1.0\end{array}$ & $\begin{array}{l}2.3 \\
2.3 \\
2.3 \\
2.3\end{array}$ & $\begin{array}{l}- \\
- \\
- \\
-\end{array}$ & $\begin{array}{l}3.3 \\
3.3 \\
3.3 \\
3.3\end{array}$ \\
\hline
\end{tabular}

Financial liabilities - continued

Shares and other equity

Excl mutual funds' shares

\begin{tabular}{|c|c|c|c|c|c|c|c|}
\hline & Quoted UK companies & $\begin{array}{r}\text { Unquoted UK } \\
\text { companies }\end{array}$ & Total & $\begin{array}{l}\text { Other accounts } \\
\text { receivable/payable }\end{array}$ & $\begin{array}{r}\text { Total financial } \\
\text { liabilities }\end{array}$ & $\begin{array}{r}\text { Total financial } \\
\text { assets }\end{array}$ & $\begin{array}{r}\text { Net assets } \\
(+) / \text { /iabilities(-) }\end{array}$ \\
\hline & F514 & F515 & F5 & F7 & $\mathrm{FL}$ & FA & BF90 \\
\hline $\begin{array}{l}2004 \\
2005 \\
2006 \\
2007 \\
2008\end{array}$ & $\begin{array}{r}\text { NNYJ } \\
14.0 \\
11.2 \\
13.5 \\
5.0 \\
0.1\end{array}$ & $\begin{array}{r}\text { NNYK } \\
119.8 \\
124.5 \\
127.7 \\
134.2 \\
151.9\end{array}$ & $\begin{array}{l}\text { NNYE } \\
133.8 \\
135.7 \\
141.2 \\
139.3 \\
152.0\end{array}$ & $\begin{array}{r}\text { NNYY } \\
4.9 \\
5.8 \\
6.3 \\
7.7 \\
26.7\end{array}$ & $\begin{array}{r}\text { NNVX } \\
4706.7 \\
5471.9 \\
7703.3 \\
9195.4 \\
15456.8\end{array}$ & $\begin{array}{r}\text { NNST } \\
4877.2 \\
5706.4 \\
8007.7 \\
9569.9 \\
15696.9\end{array}$ & $\begin{array}{l}\text { NYOL } \\
170.5 \\
234.5 \\
304.4 \\
374.5 \\
240.0\end{array}$ \\
\hline $\begin{array}{l}2009 \\
2010\end{array}$ & $\begin{array}{l}0.1 \\
0.2\end{array}$ & $\begin{array}{l}159.2 \\
163.2\end{array}$ & $\begin{array}{l}159.3 \\
163.3\end{array}$ & $\begin{array}{l}24.6 \\
25.8\end{array}$ & $\begin{array}{l}11747.9 \\
11889.4\end{array}$ & $\begin{array}{l}11959.4 \\
12192.2\end{array}$ & $\begin{array}{l}211.5 \\
302.8\end{array}$ \\
\hline $\begin{array}{r}2007 \text { Q2 } \\
\text { Q3 } \\
\text { Q4 }\end{array}$ & $\begin{array}{r}11.3 \\
6.4 \\
5.0\end{array}$ & $\begin{array}{l}128.8 \\
128.7 \\
134.2\end{array}$ & $\begin{array}{l}140.1 \\
135.1 \\
139.3\end{array}$ & $\begin{array}{l}5.8 \\
6.7 \\
7.7\end{array}$ & $\begin{array}{l}8472.4 \\
8759.7 \\
9195.4\end{array}$ & $\begin{array}{l}8842.1 \\
9161.0 \\
9569.9\end{array}$ & $\begin{array}{l}369.6 \\
401.3 \\
374.5\end{array}$ \\
\hline $\begin{array}{r}2008 \text { Q1 } \\
\text { Q2 } \\
\text { Q3 } \\
\text { Q4 }\end{array}$ & $\begin{array}{l}3.5 \\
1.8 \\
1.3 \\
0.1\end{array}$ & $\begin{array}{l}136.5 \\
138.8 \\
143.6 \\
151.9\end{array}$ & $\begin{array}{l}140.0 \\
140.6 \\
144.9 \\
152.0\end{array}$ & $\begin{array}{r}6.6 \\
6.3 \\
21.1 \\
26.7\end{array}$ & $\begin{array}{l}11161.3 \\
10522.1 \\
11349.6 \\
15456.8\end{array}$ & $\begin{array}{l}11548.4 \\
10847.4 \\
11599.3 \\
15696.9\end{array}$ & $\begin{array}{l}387.1 \\
325.3 \\
249.6 \\
240.0\end{array}$ \\
\hline $\begin{array}{r}2009 \text { Q1 } \\
\text { Q2 } \\
\text { Q3 } \\
\text { Q4 }\end{array}$ & $\begin{array}{l}0.1 \\
0.1 \\
0.1 \\
0.1\end{array}$ & $\begin{array}{l}153.5 \\
154.1 \\
157.0 \\
159.2\end{array}$ & $\begin{array}{l}153.6 \\
154.2 \\
157.1 \\
159.3\end{array}$ & $\begin{array}{l}24.9 \\
27.4 \\
25.9 \\
24.6\end{array}$ & $\begin{array}{l}15207.7 \\
12229.9 \\
12350.6 \\
11747.9\end{array}$ & $\begin{array}{l}15318.6 \\
12337.3 \\
12548.1 \\
11 \\
959.4\end{array}$ & $\begin{array}{l}110.9 \\
107.4 \\
197.5 \\
211.5\end{array}$ \\
\hline $\begin{array}{r}2010 \text { Q1 } \\
\text { Q2 } \\
\text { Q3 } \\
\text { Q4 }\end{array}$ & $\begin{array}{l}0.1 \\
0.1 \\
0.1 \\
0.2\end{array}$ & $\begin{array}{l}162.4^{\dagger} \\
163.0 \\
162.6 \\
163.2\end{array}$ & $\begin{array}{l}162.5^{\dagger} \\
163.1 \\
162.7 \\
163.3\end{array}$ & $\begin{array}{l}25.4^{\dagger} \\
28.9 \\
25.5 \\
25.8\end{array}$ & $\begin{array}{l}12444.0^{\dagger} \\
12732.2 \\
13032.2 \\
11889.4\end{array}$ & $\begin{array}{l}12741.8^{\dagger} \\
13010.4 \\
13369.0 \\
12192.2\end{array}$ & $\begin{array}{l}297.9^{\dagger} \\
278.2 \\
336.8 \\
302.8\end{array}$ \\
\hline
\end{tabular}

1 Monetary financial institutions

Sources: National Statistics

3 All loans secured on dwellings and all finance leasing are treated as long Bank of England

term loans

4 Other than direct investment loans, loans secured on dwellings and loans

for finance leasing 


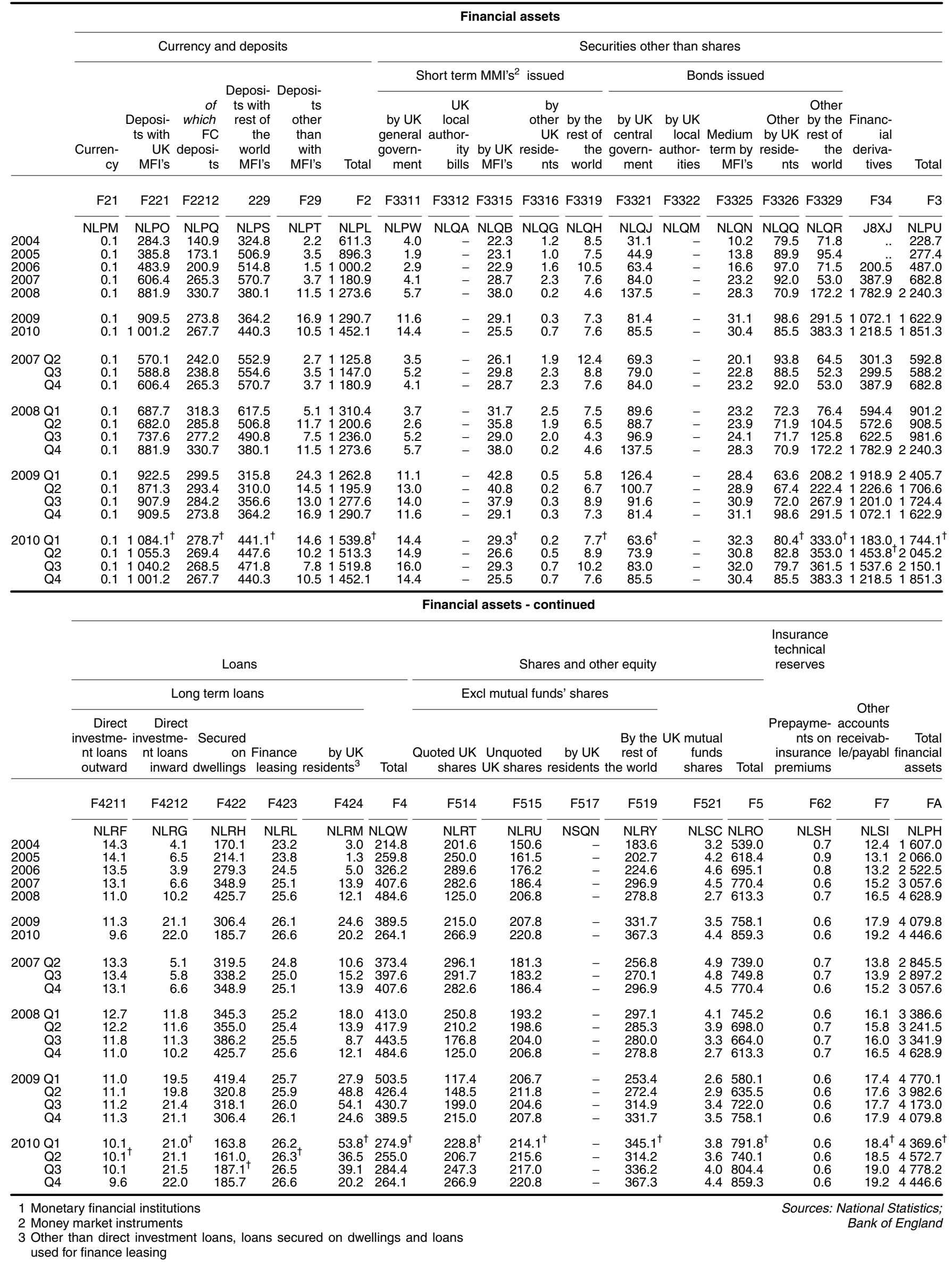




\begin{tabular}{|c|c|c|c|c|c|c|c|c|c|c|c|c|c|c|}
\hline & \multicolumn{14}{|c|}{ Financial liabilities } \\
\hline & \multicolumn{5}{|c|}{ Securities other than shares } & \multicolumn{9}{|c|}{ Loans } \\
\hline & & $\begin{array}{l}\text { Short } \\
\text { term } \\
\text { MMl's }^{1} \\
\text { issued }\end{array}$ & $\begin{array}{l}\text { Bonds } \\
\text { issued }\end{array}$ & & & \multicolumn{3}{|c|}{ Short term loans } & \multicolumn{5}{|c|}{ Long term loans } & \multirow[b]{2}{*}{ Total } \\
\hline & $\begin{array}{r}\text { Currency } \\
\text { and } \\
\text { Deposits }\end{array}$ & $\begin{array}{r}\text { by other } \\
\text { UK } \\
\text { residents }\end{array}$ & $\begin{array}{r}\text { other by } \\
\text { UK } \\
\text { residents }\end{array}$ & $\begin{array}{r}\text { Financial } \\
\text { derivati- } \\
\text { ves }\end{array}$ & Total & $\begin{array}{l}\text { Loans By } \\
\text { UK MFI's }\end{array}$ & $\begin{array}{l}\text { of which } \\
\text { FC loans } \\
\text { by UK } \\
\text { MFls }\end{array}$ & $\begin{array}{l}\text { By rest } \\
\text { of the } \\
\text { world } \\
\text { MFIs }\end{array}$ & $\begin{array}{l}\text { Direct } \\
\text { investme- } \\
\text { nt loans } \\
\text { outward }\end{array}$ & $\begin{array}{l}\text { Direct } \\
\text { investme- } \\
\text { nt loans } \\
\text { inward }\end{array}$ & $\begin{array}{c}\text { Finance } \\
\text { leasing }\end{array}$ & $\begin{array}{r}\text { by UK } \\
\text { residents }\end{array}$ & $\begin{array}{r}\text { Other by } \\
\text { the rest } \\
\text { of the } \\
\text { world }\end{array}$ & \\
\hline & AF2 & F3316 & F3326 & F34 & F3 & $\mathrm{F} 411$ & F4112 & F419 & F4211 & F4212 & $\mathrm{F} 423$ & $\mathrm{~F} 424$ & F429 & $\mathrm{F} 4$ \\
\hline $\begin{array}{l}2004 \\
2005 \\
2006 \\
2007 \\
2008\end{array}$ & $\begin{array}{r}\text { NJUF } \\
0.3 \\
1.8 \\
1.6 \\
5.9 \\
17.3\end{array}$ & $\begin{array}{r}\text { NLTK } \\
20.2 \\
26.2 \\
42.8 \\
45.4 \\
72.5\end{array}$ & $\begin{array}{l}\text { NLTU } \\
333.3 \\
430.4 \\
512.8 \\
627.9 \\
673.5\end{array}$ & $\begin{array}{r}\text { J8XK } \\
. . \\
. . \\
236.7 \\
449.5 \\
1901.1\end{array}$ & $\begin{array}{r}\text { NLSY } \\
353.5 \\
456.6 \\
792.3 \\
1122.8 \\
2647.1\end{array}$ & $\begin{array}{r}\text { NLUC } \\
475.8 \\
538.6 \\
629.7 \\
864.5 \\
1086.9\end{array}$ & $\begin{array}{l}\text { NLUE } \\
226.7 \\
253.1 \\
289.4 \\
382.7 \\
453.8\end{array}$ & $\begin{array}{l}\text { NLUG } \\
446.2 \\
670.3 \\
590.3 \\
680.0 \\
564.1\end{array}$ & $\begin{array}{r}\text { NLUJ } \\
20.6 \\
20.6 \\
20.2 \\
20.3 \\
22.2\end{array}$ & $\begin{array}{r}\text { NLUK } \\
24.0 \\
18.0 \\
18.2 \\
23.5 \\
28.8\end{array}$ & $\begin{array}{r}\text { NLUP } \\
1.6 \\
1.7 \\
1.7 \\
1.8 \\
1.9\end{array}$ & $\begin{array}{r}\text { NLUQ } \\
19.7 \\
26.2 \\
30.9 \\
30.0 \\
33.5\end{array}$ & $\begin{array}{r}\text { NLUR } \\
0.5 \\
0.5 \\
0.5 \\
0.5 \\
0.5\end{array}$ & $\begin{array}{r}\text { NLUA } \\
988.3 \\
1275.8 \\
1291.6 \\
1620.5 \\
1737.8\end{array}$ \\
\hline $\begin{array}{l}2009 \\
2010\end{array}$ & $\begin{array}{l}25.2 \\
16.3\end{array}$ & $\begin{array}{l}59.0 \\
70.4\end{array}$ & $\begin{array}{l}778.8 \\
751.8\end{array}$ & $\begin{array}{l}1125.0 \\
1257.9\end{array}$ & $\begin{array}{l}1962.7 \\
2080.1\end{array}$ & $\begin{array}{l}919.3 \\
869.3\end{array}$ & $\begin{array}{l}376.8 \\
407.1\end{array}$ & $\begin{array}{l}532.5 \\
654.7\end{array}$ & $\begin{array}{l}26.1 \\
24.8\end{array}$ & $\begin{array}{l}19.1 \\
22.9\end{array}$ & $\begin{array}{l}1.9 \\
2.0\end{array}$ & $\begin{array}{l}33.6 \\
90.4\end{array}$ & $\begin{array}{l}0.5 \\
0.5\end{array}$ & $\begin{array}{l}1533.0 \\
1664.5\end{array}$ \\
\hline $\begin{array}{r}2007 \text { Q2 } \\
\text { Q3 } \\
\text { Q4 }\end{array}$ & $\begin{array}{l}1.8 \\
2.2 \\
5.9\end{array}$ & $\begin{array}{l}47.6 \\
54.0 \\
45.4\end{array}$ & $\begin{array}{l}580.4 \\
596.6 \\
627.9\end{array}$ & $\begin{array}{l}350.5 \\
349.6 \\
449.5\end{array}$ & $\begin{array}{r}978.6 \\
1000.2 \\
1122.8\end{array}$ & $\begin{array}{l}787.0 \\
843.9 \\
864.5\end{array}$ & $\begin{array}{l}355.0 \\
359.2 \\
382.7\end{array}$ & $\begin{array}{l}706.9 \\
715.5 \\
680.0\end{array}$ & $\begin{array}{l}20.3 \\
20.3 \\
20.3\end{array}$ & $\begin{array}{l}19.1 \\
15.1 \\
23.5\end{array}$ & $\begin{array}{l}1.8 \\
1.8 \\
1.8\end{array}$ & $\begin{array}{l}31.0 \\
30.9 \\
30.0\end{array}$ & $\begin{array}{l}0.5 \\
0.5 \\
0.5\end{array}$ & $\begin{array}{l}1566.6 \\
1628.0 \\
1620.5\end{array}$ \\
\hline $\begin{array}{r}2008 \text { Q1 } \\
\text { Q2 } \\
\text { Q3 } \\
\text { Q4 }\end{array}$ & $\begin{array}{r}8.0 \\
5.9 \\
8.7 \\
17.3\end{array}$ & $\begin{array}{l}71.9 \\
67.7 \\
65.4 \\
72.5\end{array}$ & $\begin{array}{l}621.7 \\
626.8 \\
615.0 \\
673.5\end{array}$ & $\begin{array}{r}662.4 \\
633.3 \\
677.8 \\
1901.1\end{array}$ & $\begin{array}{ll}1 & 356.0 \\
1 & 327.8 \\
1 & 358.1 \\
2 & 647.1\end{array}$ & $\begin{array}{r}950.4 \\
918.2 \\
979.5 \\
1086.9\end{array}$ & $\begin{array}{l}452.9 \\
385.3 \\
398.6 \\
453.8\end{array}$ & $\begin{array}{l}803.9 \\
698.4 \\
641.3 \\
564.1\end{array}$ & $\begin{array}{l}20.9 \\
21.2 \\
22.0 \\
22.2\end{array}$ & $\begin{array}{l}28.5 \\
28.4 \\
28.6 \\
28.8\end{array}$ & $\begin{array}{l}1.8 \\
1.9 \\
1.9 \\
1.9\end{array}$ & $\begin{array}{l}36.0 \\
32.4 \\
35.5 \\
33.5\end{array}$ & $\begin{array}{l}0.5 \\
0.5 \\
0.5 \\
0.5\end{array}$ & $\begin{array}{l}1841.9 \\
1700.9 \\
1709.2 \\
1737.8\end{array}$ \\
\hline $\begin{array}{r}2009 \text { Q1 } \\
\text { Q2 } \\
\text { Q3 } \\
\text { Q4 }\end{array}$ & $\begin{array}{l}33.3 \\
23.6 \\
43.4 \\
25.2\end{array}$ & $\begin{array}{l}73.2 \\
63.1 \\
75.2 \\
59.0\end{array}$ & $\begin{array}{l}645.3 \\
659.8 \\
753.5 \\
778.8\end{array}$ & $\begin{array}{ll}2 & 009.3 \\
1 & 283.5 \\
1 & 242.8 \\
1 & 125.0\end{array}$ & $\begin{array}{ll}2 & 727.8 \\
2 & 006.4 \\
2 & 071.5 \\
1 & 962.7\end{array}$ & $\begin{array}{r}1082.9 \\
933.1 \\
921.4 \\
919.3\end{array}$ & $\begin{array}{l}406.4 \\
362.9 \\
359.8 \\
376.8\end{array}$ & $\begin{array}{l}467.7 \\
502.8 \\
585.6 \\
532.5\end{array}$ & $\begin{array}{l}26.0 \\
25.5 \\
25.0 \\
26.1\end{array}$ & $\begin{array}{l}14.6 \\
16.4 \\
17.4 \\
19.1\end{array}$ & $\begin{array}{l}1.9 \\
1.9 \\
1.9 \\
1.9\end{array}$ & $\begin{array}{l}36.1 \\
20.9 \\
27.3 \\
33.6\end{array}$ & $\begin{array}{l}0.5 \\
0.5 \\
0.5 \\
0.5\end{array}$ & $\begin{array}{l}1629.6 \\
1501.0 \\
1579.1 \\
1533.0\end{array}$ \\
\hline \multirow[t]{2}{*}{$\begin{array}{r}2010 \text { Q1 } \\
\text { Q2 } \\
\text { Q3 } \\
\text { Q4 }\end{array}$} & $\begin{array}{l}39.9 \\
29.0 \\
35.7 \\
16.3\end{array}$ & $\begin{array}{l}69.7^{\dagger} \\
61.8 \\
82.4 \\
70.4\end{array}$ & $\begin{array}{l}797.6^{\dagger} \\
750.8 \\
738.1 \\
751.8\end{array}$ & $\begin{array}{ll}1 & 229.1 \\
1 & 522.7 \\
1 & 599.0^{\dagger} \\
1 & 257.9\end{array}$ & 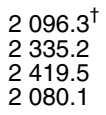 & $\begin{array}{l}930.4 \\
896.5 \\
897.1^{\dagger} \\
869.3\end{array}$ & $\begin{array}{l}410.7 \\
380.6 \\
400.5^{\dagger} \\
407.1\end{array}$ & $\begin{array}{l}609.7^{\dagger} \\
668.5 \\
687.5 \\
654.7\end{array}$ & $\begin{array}{l}24.3^{\dagger} \\
24.0 \\
23.6 \\
24.8\end{array}$ & $\begin{array}{l}23.8^{\dagger} \\
23.9^{-} \\
24.5 \\
22.9\end{array}$ & $\begin{array}{l}2.0 \\
2.0 \\
2.0 \\
2.0\end{array}$ & $\begin{array}{l}84.3^{\dagger} \\
66.4 \\
90.9 \\
90.4\end{array}$ & $\begin{array}{l}0.5 \\
0.5 \\
0.5 \\
0.5\end{array}$ & $\begin{array}{l}1674.9^{\dagger} \\
1681.8 \\
1726.2 \\
1664.5\end{array}$ \\
\hline & & & & & Financi & liabilities - & ntinued & & & & & & \multicolumn{2}{|c|}{$\begin{array}{l}\text { Net financial } \\
\text { assets/liabili- } \\
\text { ties }\end{array}$} \\
\hline
\end{tabular}

Shares and other equity

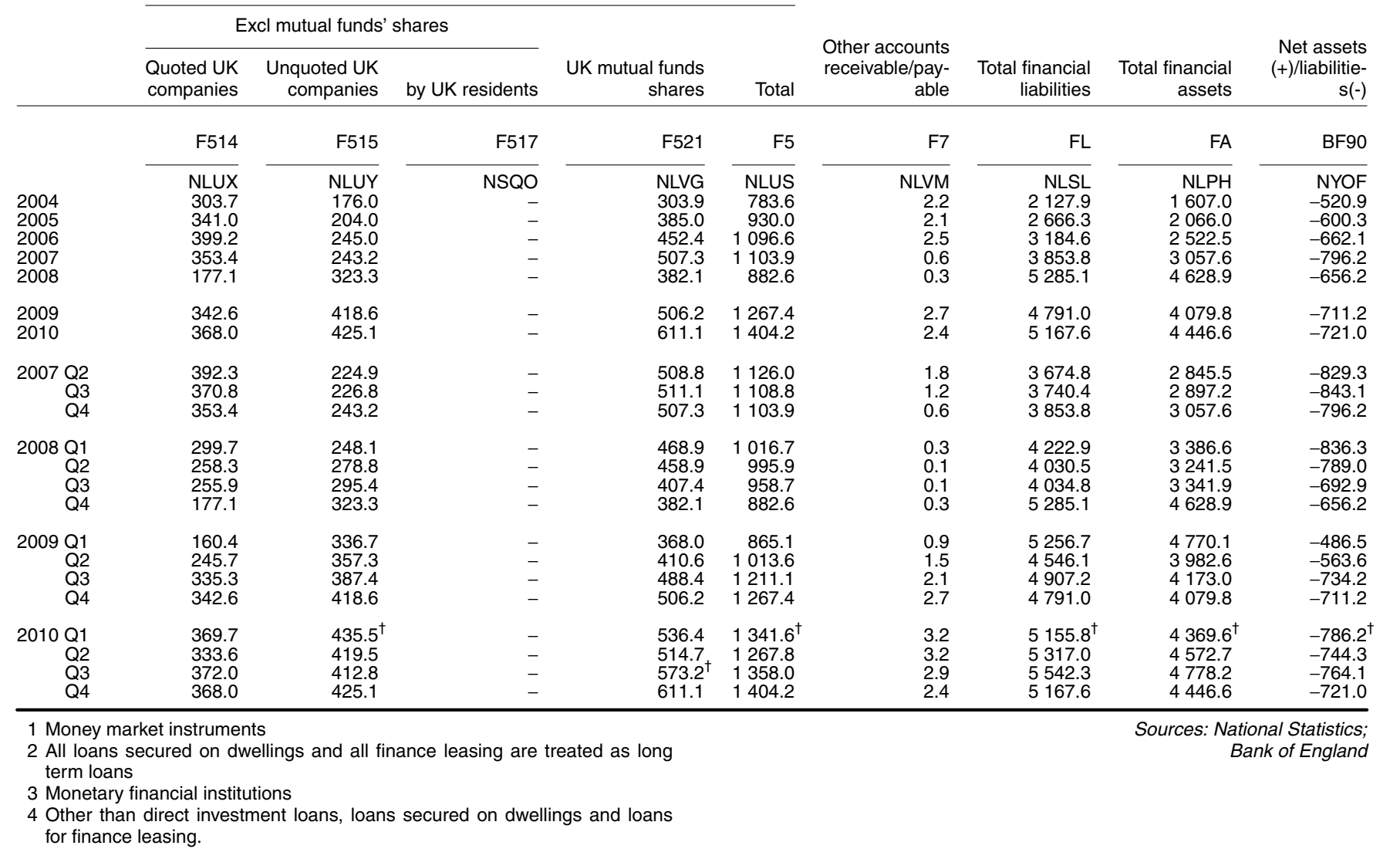




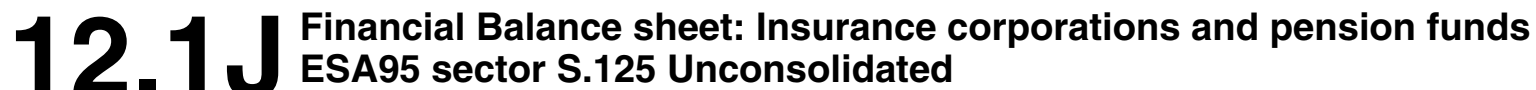

\begin{tabular}{|c|c|c|c|c|c|c|c|c|c|c|c|c|c|c|c|c|}
\hline & \multicolumn{16}{|c|}{ Financial assets } \\
\hline & \multicolumn{5}{|c|}{ Currency and deposits } & \multicolumn{11}{|c|}{ Securities other than shares } \\
\hline & \multirow[b]{2}{*}{$\begin{array}{c}\text { Deposits } \\
\text { with UK } \\
\text { MFI's }^{1}\end{array}$} & \multirow{2}{*}{$\begin{array}{r}\text { of which } \\
\text { FC } \\
\text { deposits }\end{array}$} & \multirow{2}{*}{$\begin{array}{r}\text { Deposits } \\
\text { with } \\
\text { rest of } \\
\text { the } \\
\text { world } \\
\text { MFl's }\end{array}$} & \multirow{2}{*}{$\begin{array}{r}\text { Deposits } \\
\text { other } \\
\text { than } \\
\text { with } \\
\text { MFl's }\end{array}$} & \multirow[b]{2}{*}{ Total } & \multicolumn{4}{|c|}{ Short term MMl's ${ }^{2}$ issued } & \multicolumn{5}{|c|}{ Bonds issued } & \multirow[b]{2}{*}{$\begin{array}{r}\text { Financi- } \\
\text { al } \\
\text { derivat- } \\
\text { ives }\end{array}$} & \multirow[b]{2}{*}{ Total } \\
\hline & & & & & & $\begin{array}{r}\text { by UK } \\
\text { general } \\
\text { governm- } \\
\text { ent }\end{array}$ & $\begin{array}{c}\text { by UK } \\
\text { MFI's }\end{array}$ & $\begin{array}{r}\text { by other } \\
\text { UK } \\
\text { residen- } \\
\text { ts }\end{array}$ & $\begin{array}{l}\text { by the } \\
\text { rest of } \\
\text { the } \\
\text { world }\end{array}$ & $\begin{array}{r}\text { by UK } \\
\text { central } \\
\text { governm- } \\
\text { ent }\end{array}$ & $\begin{array}{r}\text { by UK } \\
\text { local } \\
\text { authori- } \\
\text { ties }\end{array}$ & $\begin{array}{r}\text { Medium } \\
\text { term by } \\
\text { MFl's }\end{array}$ & $\begin{array}{r}\text { Other by } \\
\text { UK } \\
\text { residen- } \\
\text { ts }\end{array}$ & $\begin{array}{l}\text { by the } \\
\text { rest of } \\
\text { the } \\
\text { world }\end{array}$ & & \\
\hline & $\mathrm{F} 221$ & $\mathrm{~F} 2212$ & F229 & F29 & $\mathrm{F} 2$ & F3311 & F3315 & F3316 & F3319 & F3321 & F3322 & F3325 & F3326 & F3329 & F34 & F3 \\
\hline $\begin{array}{l}2004 \\
2005 \\
2006 \\
2007 \\
2008\end{array}$ & $\begin{array}{r}\text { NIYG } \\
64.4 \\
59.8 \\
67.6 \\
84.4 \\
82.1\end{array}$ & $\begin{array}{r}\text { IE2Y } \\
5.6 \\
4.8 \\
7.2 \\
8.0 \\
8.6\end{array}$ & $\begin{array}{r}\text { NIYK } \\
25.3 \\
33.9 \\
37.4 \\
45.4 \\
54.7\end{array}$ & $\begin{array}{r}\text { NIYL } \\
- \\
- \\
- \\
- \\
-\end{array}$ & $\begin{array}{r}\text { NIYD } \\
89.7 \\
93.7 \\
104.9 \\
129.8 \\
136.8\end{array}$ & $\begin{array}{r}\text { NIYO } \\
1.0 \\
0.4 \\
0.7 \\
0.6 \\
1.0\end{array}$ & $\begin{array}{r}\text { NIYT } \\
26.6 \\
24.9 \\
29.1 \\
30.6 \\
25.4\end{array}$ & $\begin{array}{r}\text { NIYY } \\
1.9 \\
5.5 \\
3.6 \\
5.9 \\
4.4\end{array}$ & $\begin{array}{r}\text { NIYZ } \\
2.1 \\
3.5 \\
3.0 \\
3.1 \\
3.8\end{array}$ & $\begin{array}{r}\text { NIZB } \\
224.6 \\
231.0 \\
241.1 \\
240.7 \\
238.2\end{array}$ & $\begin{array}{r}\text { NIZE } \\
0.3 \\
0.4 \\
0.5 \\
0.3 \\
0.2\end{array}$ & $\begin{array}{l}\text { NIZF } \\
29.1 \\
39.5 \\
47.9 \\
67.5 \\
82.6\end{array}$ & $\begin{array}{r}\text { NIZI } \\
175.4 \\
186.4 \\
178.2 \\
162.4 \\
144.7\end{array}$ & $\begin{array}{r}\text { NIZJ } \\
142.3 \\
168.3 \\
212.8 \\
258.0 \\
286.5\end{array}$ & $\begin{array}{r}\mathrm{J} 8 \mathrm{XL} \\
. . \\
25.0 \\
48.8 \\
148.5\end{array}$ & $\begin{array}{l}\text { NIYM } \\
603.4 \\
660.1 \\
742.0 \\
818.0 \\
935.5\end{array}$ \\
\hline $\begin{array}{l}2009 \\
2010\end{array}$ & $\begin{array}{l}89.7 \\
74.0\end{array}$ & $\begin{array}{l}7.8 \\
6.7\end{array}$ & $\begin{array}{l}43.8 \\
48.6\end{array}$ & $\begin{array}{l}- \\
-\end{array}$ & $\begin{array}{l}133.6 \\
122.6\end{array}$ & $\begin{array}{l}3.2 \\
1.2\end{array}$ & $\begin{array}{r}12.4 \\
9.7\end{array}$ & $\begin{array}{l}4.0 \\
2.3\end{array}$ & $\begin{array}{l}3.2 \\
4.2\end{array}$ & $\begin{array}{l}250.4 \\
295.4\end{array}$ & $\begin{array}{l}0.5 \\
0.6\end{array}$ & $\begin{array}{l}91.0 \\
88.5\end{array}$ & $\begin{array}{l}165.6 \\
140.0\end{array}$ & $\begin{array}{l}333.1 \\
379.2\end{array}$ & $\begin{array}{l}96.6 \\
82.0\end{array}$ & $\begin{array}{r}960.0 \\
1003.0\end{array}$ \\
\hline $\begin{array}{r}2007 \text { Q2 } \\
\text { Q3 } \\
\text { Q4 }\end{array}$ & $\begin{array}{l}68.9 \\
70.9 \\
84.4\end{array}$ & $\begin{array}{l}6.2 \\
7.1 \\
8.0\end{array}$ & $\begin{array}{l}42.6 \\
44.2 \\
45.4\end{array}$ & $\begin{array}{l}- \\
- \\
-\end{array}$ & $\begin{array}{l}111.5 \\
115.1 \\
129.8\end{array}$ & $\begin{array}{l}0.3 \\
0.3 \\
0.6\end{array}$ & $\begin{array}{l}27.6 \\
31.8 \\
30.6\end{array}$ & $\begin{array}{l}5.5 \\
7.3 \\
5.9\end{array}$ & $\begin{array}{l}2.8 \\
3.6 \\
3.1\end{array}$ & $\begin{array}{l}228.8 \\
235.7 \\
240.7\end{array}$ & $\begin{array}{l}0.4 \\
0.3 \\
0.3\end{array}$ & $\begin{array}{l}58.3 \\
66.4 \\
67.5\end{array}$ & $\begin{array}{l}165.3 \\
160.6 \\
162.4\end{array}$ & $\begin{array}{l}229.3 \\
239.4 \\
258.0\end{array}$ & $\begin{array}{l}33.1 \\
32.5 \\
48.8\end{array}$ & $\begin{array}{l}751.4 \\
777.9 \\
818.0\end{array}$ \\
\hline $\begin{array}{r}2008 \text { Q1 } \\
\text { Q2 } \\
\text { Q3 } \\
\text { Q4 }\end{array}$ & $\begin{array}{l}91.2 \\
90.1 \\
87.2 \\
82.1\end{array}$ & $\begin{array}{r}10.2 \\
10.2 \\
8.9 \\
8.6\end{array}$ & $\begin{array}{l}52.4 \\
49.6 \\
50.6 \\
54.7\end{array}$ & $\begin{array}{l}- \\
- \\
- \\
-\end{array}$ & $\begin{array}{l}143.6 \\
139.6 \\
137.8 \\
136.8\end{array}$ & $\begin{array}{l}0.8 \\
0.7 \\
2.1 \\
1.0\end{array}$ & $\begin{array}{l}26.8 \\
26.0 \\
29.6 \\
25.4\end{array}$ & $\begin{array}{l}3.8 \\
4.7 \\
5.9 \\
4.4\end{array}$ & $\begin{array}{l}3.6 \\
4.1 \\
4.5 \\
3.8\end{array}$ & $\begin{array}{l}242.3 \\
237.0 \\
222.4 \\
238.2\end{array}$ & $\begin{array}{l}0.3 \\
0.3 \\
0.3 \\
0.2\end{array}$ & $\begin{array}{l}67.5 \\
69.6 \\
70.2 \\
82.6\end{array}$ & $\begin{array}{l}159.0 \\
152.7 \\
149.3 \\
144.7\end{array}$ & $\begin{array}{l}247.7 \\
237.8 \\
245.9 \\
286.5\end{array}$ & $\begin{array}{r}70.0 \\
67.1 \\
66.0 \\
148.5\end{array}$ & $\begin{array}{l}821.7 \\
800.0 \\
796.2 \\
935.5\end{array}$ \\
\hline $\begin{array}{r}2009 \text { Q1 } \\
\text { Q2 } \\
\text { Q3 } \\
\text { Q4 }\end{array}$ & $\begin{array}{l}82.3 \\
89.3 \\
83.6 \\
89.7\end{array}$ & $\begin{array}{r}8.4 \\
9.1 \\
11.2 \\
7.8\end{array}$ & $\begin{array}{l}46.0 \\
44.6 \\
46.4 \\
43.8\end{array}$ & $\begin{array}{l}- \\
- \\
- \\
-\end{array}$ & $\begin{array}{l}128.3 \\
134.0 \\
130.0 \\
133.6\end{array}$ & $\begin{array}{l}1.4 \\
1.2 \\
2.4 \\
3.2\end{array}$ & $\begin{array}{l}21.6 \\
16.8 \\
14.0 \\
12.4\end{array}$ & $\begin{array}{l}3.5 \\
4.5 \\
5.3 \\
4.0\end{array}$ & $\begin{array}{l}2.5 \\
2.4 \\
2.7 \\
3.2\end{array}$ & $\begin{array}{l}229.4 \\
233.8 \\
250.4 \\
250.4\end{array}$ & $\begin{array}{l}0.4 \\
0.5 \\
0.6 \\
0.5\end{array}$ & $\begin{array}{l}82.8 \\
84.2 \\
90.3 \\
91.0\end{array}$ & $\begin{array}{l}148.4 \\
162.3 \\
170.3 \\
165.6\end{array}$ & $\begin{array}{l}302.3 \\
316.9 \\
351.0 \\
333.1\end{array}$ & $\begin{array}{r}153.7 \\
113.0 \\
95.8 \\
96.6\end{array}$ & $\begin{array}{l}945.9 \\
935.6 \\
982.9 \\
960.0\end{array}$ \\
\hline $\begin{array}{r}2010 \text { Q1 } \\
\text { Q2 } \\
\text { Q3 } \\
\text { Q4 }\end{array}$ & $\begin{array}{l}88.2^{\dagger} \\
83.4 \\
75.8 \\
74.0\end{array}$ & $\begin{array}{l}8.2^{\dagger} \\
7.6 \\
6.5 \\
6.7\end{array}$ & $\begin{array}{l}46.8^{\dagger} \\
47.9 \\
49.1 \\
48.6\end{array}$ & $\begin{array}{l}- \\
- \\
- \\
-\end{array}$ & $\begin{array}{l}135.0^{\dagger} \\
131.2 \\
124.9 \\
122.6\end{array}$ & $\begin{array}{l}3.1 \\
2.1 \\
1.1 \\
1.2\end{array}$ & $\begin{array}{c}11.0^{\dagger} \\
9.6 \\
10.3 \\
9.7\end{array}$ & $\begin{array}{l}4.0^{\dagger} \\
3.5 \\
2.7 \\
2.3\end{array}$ & $\begin{array}{l}4.7^{\dagger} \\
4.5 \\
4.7 \\
4.2\end{array}$ & $\begin{array}{l}275.3^{\dagger} \\
279.0 \\
298.7 \\
295.4\end{array}$ & $\begin{array}{l}0.5 \\
0.5 \\
0.5 \\
0.6\end{array}$ & $\begin{array}{l}94.5 \\
89.9 \\
93.3 \\
88.5\end{array}$ & $\begin{array}{l}164.3^{\dagger} \\
155.8 \\
135.4 \\
140.0\end{array}$ & $\begin{array}{l}367.6^{\dagger} \\
353.9 \\
365.1 \\
379.2\end{array}$ & $\begin{array}{r}87.3^{\dagger} \\
104.4 \\
102.5 \\
82.0\end{array}$ & $\begin{array}{ll}\dagger & 012.4^{\dagger} \\
1 & 003.3 \\
1 & 014.2 \\
1 & 003.0\end{array}$ \\
\hline
\end{tabular}

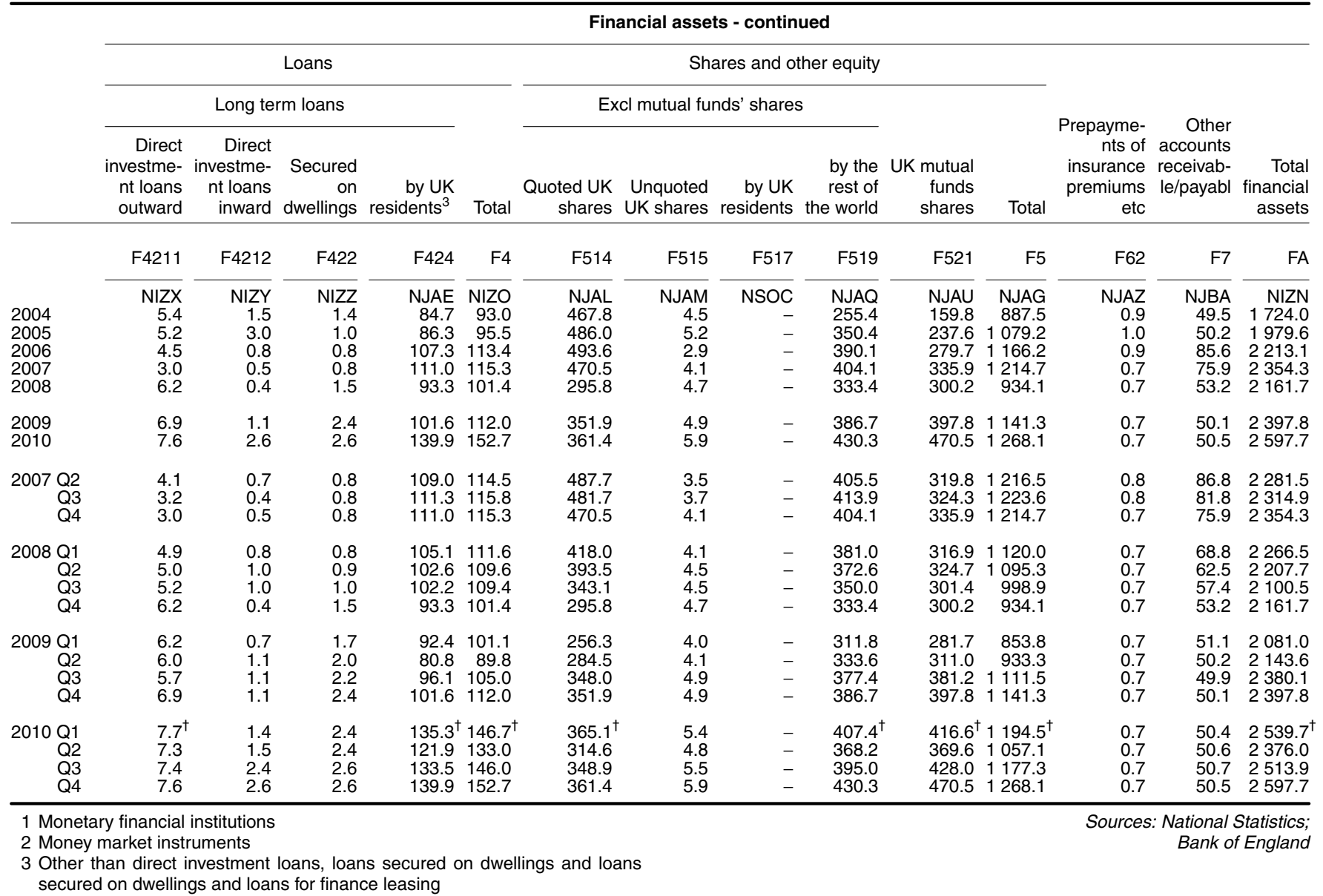




\section{$12.1 \mathrm{~J}$.

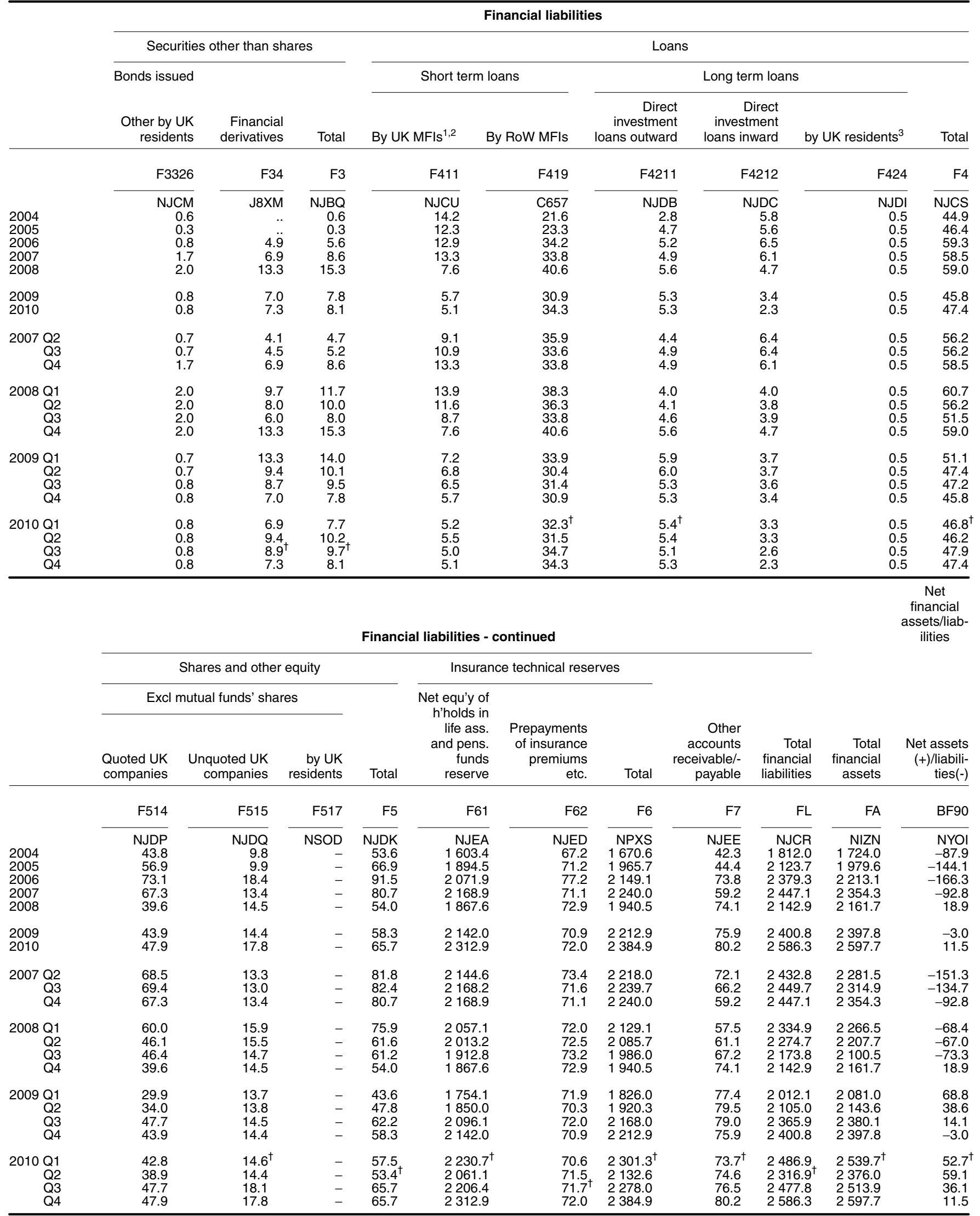

1 All loans secured on dwellings and all finance leasing are treated as long term loans

Sources: National Statistics;

2 Monetary financial institutions

3 Other than direct investment loans, loans secured on dwellings and loans

for finance leasing 


\begin{tabular}{|c|c|c|c|c|c|c|c|c|c|c|c|c|c|c|c|c|}
\hline & \multicolumn{16}{|c|}{ Financial assets } \\
\hline & \multirow[b]{3}{*}{$\begin{array}{r}\text { Monetary } \\
\text { gold and } \\
\text { SDRs }\end{array}$} & \multicolumn{4}{|c|}{ Currency and deposits } & \multicolumn{11}{|c|}{ Securities other than shares } \\
\hline & & \multirow[b]{2}{*}{$\begin{array}{c}\text { Deposits } \\
\text { with UK } \\
\text { MFI'S }^{1}\end{array}$} & \multirow{2}{*}{$\begin{array}{r}\text { Deposits } \\
\text { with } \\
\text { rest of } \\
\text { the } \\
\text { world } \\
\text { MFI's }\end{array}$} & \multirow[b]{2}{*}{$\begin{array}{r}\text { Deposits } \\
\text { other } \\
\text { than } \\
\text { with } \\
\text { MFl's }\end{array}$} & \multirow[b]{2}{*}{ Total } & \multicolumn{5}{|c|}{ Short term MMl's ${ }^{2}$ issued } & \multicolumn{4}{|c|}{ Bonds issued } & \multirow[b]{2}{*}{$\begin{array}{r}\text { Financi- } \\
\text { al } \\
\text { derivat- } \\
\text { ives }\end{array}$} & \multirow[b]{2}{*}{ Total } \\
\hline & & & & & & $\begin{array}{r}\text { by UK } \\
\text { general } \\
\text { governm- } \\
\text { ent }\end{array}$ & $\begin{array}{c}\text { UK local } \\
\text { authori- } \\
\text { ty bills }\end{array}$ & $\begin{array}{c}\text { by UK } \\
\text { MFI's }\end{array}$ & $\begin{array}{r}\text { by other } \\
\text { UK } \\
\text { residen- } \\
\text { ts }\end{array}$ & $\begin{array}{r}\text { by the } \\
\text { rest of } \\
\text { the } \\
\text { world }\end{array}$ & $\begin{array}{r}\text { by UK } \\
\text { central } \\
\text { governm- } \\
\text { ent }\end{array}$ & $\begin{array}{r}\text { by UK } \\
\text { local } \\
\text { authori- } \\
\text { ties }\end{array}$ & $\begin{array}{r}\text { Other by } \\
\text { UK } \\
\text { residen- } \\
\text { ts }\end{array}$ & $\begin{array}{l}\text { by the } \\
\text { rest of } \\
\text { the } \\
\text { world }\end{array}$ & & \\
\hline & $\mathrm{F} 1$ & F221 & F229 & F29 & $\mathrm{F} 2$ & F3311 & F3312 & F3315 & F3316 & F3319 & F3321 & F3322 & F3326 & F3329 & F34 & F3 \\
\hline $\begin{array}{l}2004 \\
2005 \\
2006 \\
2007 \\
2008\end{array}$ & $\begin{array}{r}\text { NIFC } \\
2.5 \\
3.2 \\
3.4 \\
4.3 \\
6.3\end{array}$ & $\begin{array}{r}\text { NLVW } \\
26.9 \\
27.1 \\
34.6 \\
40.9 \\
57.4\end{array}$ & $\begin{array}{r}\text { NLWA } \\
3.3 \\
2.0 \\
1.2 \\
1.0 \\
4.6\end{array}$ & $\begin{array}{r}\text { NLWB } \\
0.4 \\
0.4 \\
0.2 \\
4.6 \\
17.7\end{array}$ & $\begin{array}{r}\text { NLUT } \\
30.6 \\
29.4 \\
35.9 \\
46.5 \\
79.7\end{array}$ & $\begin{array}{r}\text { NLWE } \\
0.1 \\
0.1 \\
0.1 \\
0.1 \\
0.1\end{array}$ & $\begin{array}{r}\text { NLWI } \\
- \\
- \\
- \\
- \\
-\end{array}$ & $\begin{array}{r}\text { NLWJ } \\
4.4 \\
4.5 \\
5.2 \\
2.3 \\
3.9\end{array}$ & $\begin{array}{r}\text { NLWO } \\
0.2 \\
0.3 \\
2.1 \\
1.3 \\
1.5\end{array}$ & $\begin{array}{r}\text { NLWP } \\
0.3 \\
1.7 \\
3.0 \\
5.5 \\
5.3\end{array}$ & $\begin{array}{r}\text { NLWR } \\
0.2 \\
0.2 \\
0.2 \\
0.1 \\
0.2\end{array}$ & $\begin{array}{r}\text { NLWU } \\
- \\
- \\
- \\
- \\
-\end{array}$ & $\begin{array}{r}\text { NLWY } \\
0.1 \\
1.1 \\
0.5 \\
0.5 \\
5.5\end{array}$ & $\begin{array}{r}\text { NLWZ } \\
17.1 \\
17.5 \\
15.3 \\
18.2 \\
24.4\end{array}$ & $\begin{array}{r}\text { ZYBQ } \\
0.2 \\
0.6 \\
0.7 \\
-0.4 \\
-3.5\end{array}$ & $\begin{array}{r}\text { NLWC } \\
22.5 \\
26.0 \\
27.2 \\
27.6 \\
37.4\end{array}$ \\
\hline $\begin{array}{l}2009 \\
2010\end{array}$ & $\begin{array}{l}15.7 \\
18.2\end{array}$ & $\begin{array}{l}57.2 \\
30.6\end{array}$ & $\begin{array}{l}4.9 \\
6.0\end{array}$ & $\begin{array}{l}26.8 \\
15.7\end{array}$ & $\begin{array}{l}88.9 \\
52.3\end{array}$ & $\begin{array}{l}0.1 \\
0.9\end{array}$ & $\begin{array}{l}- \\
-\end{array}$ & $\begin{array}{l}1.6 \\
1.0\end{array}$ & $\begin{array}{l}1.4 \\
2.8\end{array}$ & $\begin{array}{l}5.6 \\
6.1\end{array}$ & $\begin{array}{l}0.2 \\
0.2\end{array}$ & $\begin{array}{l}- \\
-\end{array}$ & $\begin{array}{l}0.3 \\
0.3\end{array}$ & $\begin{array}{l}21.1 \\
27.1\end{array}$ & $\begin{array}{l}-1.4 \\
-0.6\end{array}$ & $\begin{array}{l}29.0 \\
37.9\end{array}$ \\
\hline $\begin{array}{r}2007 \text { Q2 } \\
\text { Q3 } \\
\text { Q4 }\end{array}$ & $\begin{array}{l}3.4 \\
3.8 \\
4.3\end{array}$ & $\begin{array}{l}41.0 \\
47.1 \\
40.9\end{array}$ & $\begin{array}{l}0.8 \\
0.9 \\
1.0\end{array}$ & $\begin{array}{l}0.4 \\
0.8 \\
4.6\end{array}$ & $\begin{array}{l}42.2 \\
48.8 \\
46.5\end{array}$ & $\begin{array}{l}0.1 \\
0.1 \\
0.1\end{array}$ & $\begin{array}{l}- \\
- \\
-\end{array}$ & $\begin{array}{l}7.4 \\
3.8 \\
2.3\end{array}$ & $\begin{array}{l}1.3 \\
1.4 \\
1.3\end{array}$ & $\begin{array}{l}4.0 \\
5.5 \\
5.5\end{array}$ & $\begin{array}{l}0.2 \\
0.2 \\
0.1\end{array}$ & $\begin{array}{l}- \\
- \\
-\end{array}$ & $\begin{array}{l}0.5 \\
0.5 \\
0.5\end{array}$ & $\begin{array}{l}14.0 \\
16.1 \\
18.2\end{array}$ & $\begin{array}{r}0.7 \\
0.2 \\
-0.4\end{array}$ & $\begin{array}{l}28.2 \\
27.7 \\
27.6\end{array}$ \\
\hline $\begin{array}{r}2008 \text { Q1 } \\
\text { Q2 } \\
\text { Q3 } \\
\text { Q4 }\end{array}$ & $\begin{array}{l}4.9 \\
4.9 \\
5.2 \\
6.3\end{array}$ & $\begin{array}{l}36.5 \\
40.4 \\
60.5 \\
57.4\end{array}$ & $\begin{array}{l}1.9 \\
2.4 \\
1.8 \\
4.6\end{array}$ & $\begin{array}{r}6.6 \\
4.6 \\
7.6 \\
17.7\end{array}$ & $\begin{array}{l}45.1 \\
47.4 \\
69.9 \\
79.7\end{array}$ & $\begin{array}{l}0.1 \\
\overline{-} \\
0.1 \\
0.1\end{array}$ & $\begin{array}{l}- \\
- \\
- \\
-\end{array}$ & $\begin{array}{l}1.7 \\
1.7 \\
1.3 \\
3.9\end{array}$ & $\begin{array}{l}1.5 \\
1.5 \\
1.9 \\
1.5\end{array}$ & $\begin{array}{l}8.7 \\
1.4 \\
7.1 \\
5.3\end{array}$ & $\begin{array}{l}0.1 \\
0.2 \\
0.2 \\
0.2\end{array}$ & $\begin{array}{l}- \\
- \\
- \\
-\end{array}$ & $\begin{array}{l}0.5 \\
0.5 \\
0.5 \\
5.5\end{array}$ & $\begin{array}{l}19.9 \\
18.8 \\
18.9 \\
24.4\end{array}$ & $\begin{array}{l}-1.3 \\
-0.9 \\
-1.1 \\
-3.5\end{array}$ & $\begin{array}{l}31.2 \\
23.2 \\
28.8 \\
37.4\end{array}$ \\
\hline $\begin{array}{r}2009 \text { Q1 } \\
\text { Q2 } \\
\text { Q3 } \\
\text { Q4 }\end{array}$ & $\begin{array}{r}6.7 \\
5.9 \\
14.9 \\
15.7\end{array}$ & $\begin{array}{l}50.8 \\
55.6 \\
57.9 \\
57.2\end{array}$ & $\begin{array}{l}4.3 \\
4.9 \\
5.4 \\
4.9\end{array}$ & $\begin{array}{l}34.8 \\
26.9 \\
47.0 \\
26.8\end{array}$ & $\begin{array}{r}89.9 \\
87.4 \\
110.3 \\
88.9\end{array}$ & $\begin{array}{l}0.1 \\
0.1 \\
0.1 \\
0.1\end{array}$ & $\begin{array}{l}- \\
- \\
- \\
-\end{array}$ & $\begin{array}{l}3.6 \\
3.7 \\
3.6 \\
1.6\end{array}$ & $\begin{array}{l}1.1 \\
1.9 \\
1.6 \\
1.4\end{array}$ & $\begin{array}{l}5.8 \\
9.6 \\
7.0 \\
5.6\end{array}$ & $\begin{array}{l}0.2 \\
0.2 \\
0.2 \\
0.2\end{array}$ & $\begin{array}{l}- \\
- \\
- \\
-\end{array}$ & $\begin{array}{l}9.3 \\
0.3 \\
0.3 \\
0.3\end{array}$ & $\begin{array}{l}23.7 \\
21.3 \\
21.8 \\
21.1\end{array}$ & $\begin{array}{l}-2.8 \\
-1.4 \\
-1.6 \\
-1.4\end{array}$ & $\begin{array}{l}40.9 \\
35.6 \\
33.0 \\
29.0\end{array}$ \\
\hline $\begin{array}{r}2010 \text { Q1 } \\
\text { Q2 } \\
\text { Q3 } \\
\text { Q4 }\end{array}$ & $\begin{array}{l}16.5 \\
17.3 \\
17.3 \\
18.2\end{array}$ & $\begin{array}{l}41.1 \\
41.6 \\
33.3 \\
30.6\end{array}$ & $\begin{array}{l}6.4 \\
6.2 \\
6.9 \\
6.0\end{array}$ & $\begin{array}{l}40.9 \\
29.0 \\
35.5 \\
15.7\end{array}$ & $\begin{array}{l}88.5 \\
76.7 \\
75.7 \\
52.3\end{array}$ & $\begin{array}{l}0.2 \\
1.4 \\
1.2 \\
0.9\end{array}$ & $\begin{array}{l}- \\
- \\
- \\
-\end{array}$ & $\begin{array}{l}1.1 \\
1.0 \\
1.1 \\
1.0\end{array}$ & $\begin{array}{l}0.9 \\
2.2 \\
2.2 \\
2.8\end{array}$ & $\begin{array}{r}4.0 \\
7.8 \\
11.4 \\
6.1\end{array}$ & $\begin{array}{l}0.2 \\
0.2 \\
0.2 \\
0.2\end{array}$ & $\begin{array}{l}- \\
- \\
- \\
-\end{array}$ & $\begin{array}{l}0.3 \\
0.3 \\
0.3 \\
0.3\end{array}$ & $\begin{array}{l}22.4 \\
24.0 \\
25.3 \\
27.1\end{array}$ & $\begin{array}{l}-1.0 \\
-0.5 \\
-0.6 \\
-0.6\end{array}$ & $\begin{array}{l}28.1 \\
36.5 \\
41.0 \\
37.9\end{array}$ \\
\hline
\end{tabular}

\begin{tabular}{|c|c|c|c|c|c|c|c|c|c|c|c|}
\hline & \multicolumn{11}{|c|}{ Financial assets - continued } \\
\hline & \multicolumn{3}{|c|}{ Loans } & \multicolumn{5}{|c|}{ Shares and other equity } & \multirow{3}{*}{$\begin{array}{l}\text { Insurance } \\
\text { technical } \\
\text { reserves } \\
\text { Prepayments } \\
\text { of insurance } \\
\text { premiums } \\
\text { etc. }\end{array}$} & \multirow{3}{*}{$\begin{array}{r}\text { Other } \\
\text { accounts } \\
\text { receivable/- } \\
\text { payable }\end{array}$} & \multirow{3}{*}{$\begin{array}{r}\text { Total } \\
\text { financial } \\
\text { assets } \\
\end{array}$} \\
\hline & \multicolumn{2}{|c|}{ Long term loans } & \multirow[b]{2}{*}{ Total } & \multicolumn{4}{|c|}{ Excl mutual funds' shares } & \multirow[b]{2}{*}{ Total } & & & \\
\hline & $\begin{array}{r}\text { Secured on } \\
\text { dwellings }\end{array}$ & $\begin{array}{l}\text { by UK } \\
\text { residents }^{3}\end{array}$ & & $\begin{array}{r}\text { Quoted UK } \\
\text { shares }\end{array}$ & $\begin{array}{r}\text { Unquoted UK } \\
\text { shares }\end{array}$ & $\begin{array}{r}\text { Other UK } \\
\text { equity }\end{array}$ & $\begin{array}{l}\text { by the rest } \\
\text { of the world }\end{array}$ & & & & \\
\hline & $\mathrm{F} 422$ & $\mathrm{~F} 424$ & $\mathrm{~F} 4$ & $\mathrm{~F} 514$ & F515 & F516 & F519 & F5 & F62 & $\mathrm{F} 7$ & FA \\
\hline $\begin{array}{l}2004 \\
2005 \\
2006 \\
2007 \\
2008\end{array}$ & $\begin{array}{r}\text { NLXP } \\
1.1 \\
1.4 \\
1.7 \\
2.2 \\
3.4\end{array}$ & $\begin{array}{r}\mathrm{NLXU} \\
69.6 \\
74.5 \\
78.0 \\
83.6 \\
89.4\end{array}$ & $\begin{array}{r}\text { NLXE } \\
70.7 \\
75.9 \\
79.6 \\
85.8 \\
92.8\end{array}$ & $\begin{array}{r}\text { NLYB } \\
0.8 \\
1.1 \\
1.6 \\
1.4 \\
13.1\end{array}$ & $\begin{array}{r}\text { NLYC } \\
2.1 \\
2.1 \\
4.7 \\
3.0 \\
6.7\end{array}$ & $\begin{array}{c}\mathrm{H} 4 \mathrm{O} 9 \\
113.3 \\
129.5 \\
120.7 \\
119.1 \\
122.3\end{array}$ & $\begin{array}{r}\text { NLYG } \\
8.0 \\
8.7 \\
9.5 \\
10.2 \\
11.2\end{array}$ & $\begin{array}{r}\text { NLXW } \\
124.2 \\
141.4 \\
136.5 \\
133.8 \\
153.3\end{array}$ & $\begin{array}{r}\text { NLYP } \\
0.8 \\
0.8 \\
0.8 \\
0.9 \\
0.9\end{array}$ & $\begin{array}{r}\text { NLYQ } \\
47.5 \\
53.4 \\
56.2 \\
55.2 \\
71.6\end{array}$ & $\begin{array}{r}\text { NPUP } \\
298.8 \\
330.1 \\
339.7 \\
354.1 \\
442.0\end{array}$ \\
\hline $\begin{array}{l}2009 \\
2010\end{array}$ & $\begin{array}{l}4.1 \\
4.9\end{array}$ & $\begin{array}{r}92.3 \\
100.9\end{array}$ & $\begin{array}{r}96.4 \\
105.8\end{array}$ & $\begin{array}{l}54.6 \\
55.1\end{array}$ & $\begin{array}{l}6.6 \\
6.5\end{array}$ & $\begin{array}{l}117.7 \\
116.8\end{array}$ & $\begin{array}{l}12.3 \\
13.5\end{array}$ & $\begin{array}{l}191.1 \\
192.0\end{array}$ & $\begin{array}{l}0.9 \\
0.9\end{array}$ & $\begin{array}{l}72.4 \\
76.2\end{array}$ & $\begin{array}{l}494.4 \\
483.2\end{array}$ \\
\hline $\begin{array}{r}2007 \text { Q2 } \\
\text { Q3 } \\
\text { Q4 }\end{array}$ & $\begin{array}{l}1.8 \\
2.0 \\
2.2\end{array}$ & $\begin{array}{l}78.3 \\
81.9 \\
83.6\end{array}$ & $\begin{array}{l}80.1 \\
84.0 \\
85.8\end{array}$ & $\begin{array}{l}1.5 \\
1.5 \\
1.4\end{array}$ & $\begin{array}{l}3.3 \\
3.0 \\
3.0\end{array}$ & $\begin{array}{l}116.7 \\
118.7 \\
119.1\end{array}$ & $\begin{array}{l}10.2 \\
10.2 \\
10.2\end{array}$ & $\begin{array}{l}131.8 \\
133.4 \\
133.8\end{array}$ & $\begin{array}{l}0.8 \\
0.8 \\
0.9\end{array}$ & $\begin{array}{l}58.8 \\
56.2 \\
55.2\end{array}$ & $\begin{array}{l}345.3 \\
354.7 \\
354.1\end{array}$ \\
\hline $\begin{array}{r}2008 \text { Q1 } \\
\text { Q2 } \\
\text { Q3 } \\
\text { Q4 }\end{array}$ & $\begin{array}{l}2.7 \\
3.1 \\
3.2 \\
3.4\end{array}$ & $\begin{array}{l}85.3 \\
86.4 \\
87.9 \\
89.4\end{array}$ & $\begin{array}{l}88.0 \\
89.5 \\
91.1 \\
92.8\end{array}$ & $\begin{array}{r}1.3 \\
1.3 \\
1.0 \\
13.1\end{array}$ & $\begin{array}{l}3.0 \\
3.1 \\
6.6 \\
6.7\end{array}$ & $\begin{array}{l}119.4 \\
121.3 \\
121.9 \\
122.3\end{array}$ & $\begin{array}{l}10.3 \\
10.8 \\
11.0 \\
11.2\end{array}$ & $\begin{array}{l}134.0 \\
136.4 \\
140.4 \\
153.3\end{array}$ & $\begin{array}{l}0.9 \\
0.9 \\
0.9 \\
0.9\end{array}$ & $\begin{array}{l}62.0 \\
58.1 \\
67.6 \\
71.6\end{array}$ & $\begin{array}{l}366.0 \\
360.3 \\
403.9 \\
442.0\end{array}$ \\
\hline $\begin{array}{r}2009 \text { Q1 } \\
\text { Q2 } \\
\text { Q3 } \\
\text { Q4 }\end{array}$ & $\begin{array}{l}3.6 \\
4.0 \\
4.1 \\
4.1\end{array}$ & $\begin{array}{l}89.6 \\
90.8 \\
91.8 \\
92.3\end{array}$ & $\begin{array}{l}93.2 \\
94.8 \\
95.8 \\
96.4\end{array}$ & $\begin{array}{l}22.2 \\
29.2 \\
29.3 \\
54.6\end{array}$ & $\begin{array}{l}6.7 \\
6.7 \\
6.6 \\
6.6\end{array}$ & $\begin{array}{l}117.9 \\
117.9 \\
117.7 \\
117.7\end{array}$ & $\begin{array}{l}11.2 \\
12.0 \\
12.1 \\
12.3\end{array}$ & $\begin{array}{l}157.9 \\
165.8 \\
165.7 \\
191.1\end{array}$ & $\begin{array}{l}0.9 \\
0.8 \\
0.9 \\
0.9\end{array}$ & $\begin{array}{l}75.3 \\
74.8 \\
72.6 \\
72.4\end{array}$ & $\begin{array}{l}464.8 \\
465.2 \\
493.2 \\
494.4\end{array}$ \\
\hline $\begin{array}{r}2010 \text { Q1 } \\
\text { Q2 } \\
\text { Q3 } \\
\text { Q4 }\end{array}$ & $\begin{array}{l}4.6 \\
4.8 \\
4.8^{\dagger} \\
4.9\end{array}$ & $\begin{array}{r}94.3 \\
99.0 \\
100.2 \\
100.9\end{array}$ & $\begin{array}{r}98.9 \\
103.8 \\
105.1 \\
105.8\end{array}$ & $\begin{array}{l}54.2 \\
54.5 \\
54.8 \\
55.1\end{array}$ & $\begin{array}{l}6.5 \\
6.5 \\
6.5 \\
6.5\end{array}$ & $\begin{array}{l}117.1^{\dagger} \\
116.8 \\
116.5 \\
116.8\end{array}$ & $\begin{array}{l}12.3 \\
12.4 \\
13.1 \\
13.5\end{array}$ & $\begin{array}{l}190.1^{\dagger} \\
190.4 \\
191.0 \\
192.0\end{array}$ & $\begin{array}{l}0.8 \\
0.9 \\
0.9 \\
0.9\end{array}$ & $\begin{array}{l}85.9^{\dagger} \\
83.7 \\
75.6 \\
76.2\end{array}$ & $\begin{array}{l}508.9^{\dagger} \\
509.3 \\
506.5 \\
483.2\end{array}$ \\
\hline $\begin{array}{l}1 \text { Monete } \\
2 \text { Money } \\
3 \text { Other t } \\
\text { used fo }\end{array}$ & $\begin{array}{l}\text { lancial institut } \\
\text { et instrument } \\
\text { direct investm } \\
\text { nce leasing }\end{array}$ & ns & & on dwellin & and loans & & & & & $\begin{array}{r}\text { ces: National } \\
\text { Bank }\end{array}$ & $\begin{array}{l}\text { Statistics; } \\
\text { f England }\end{array}$ \\
\hline
\end{tabular}




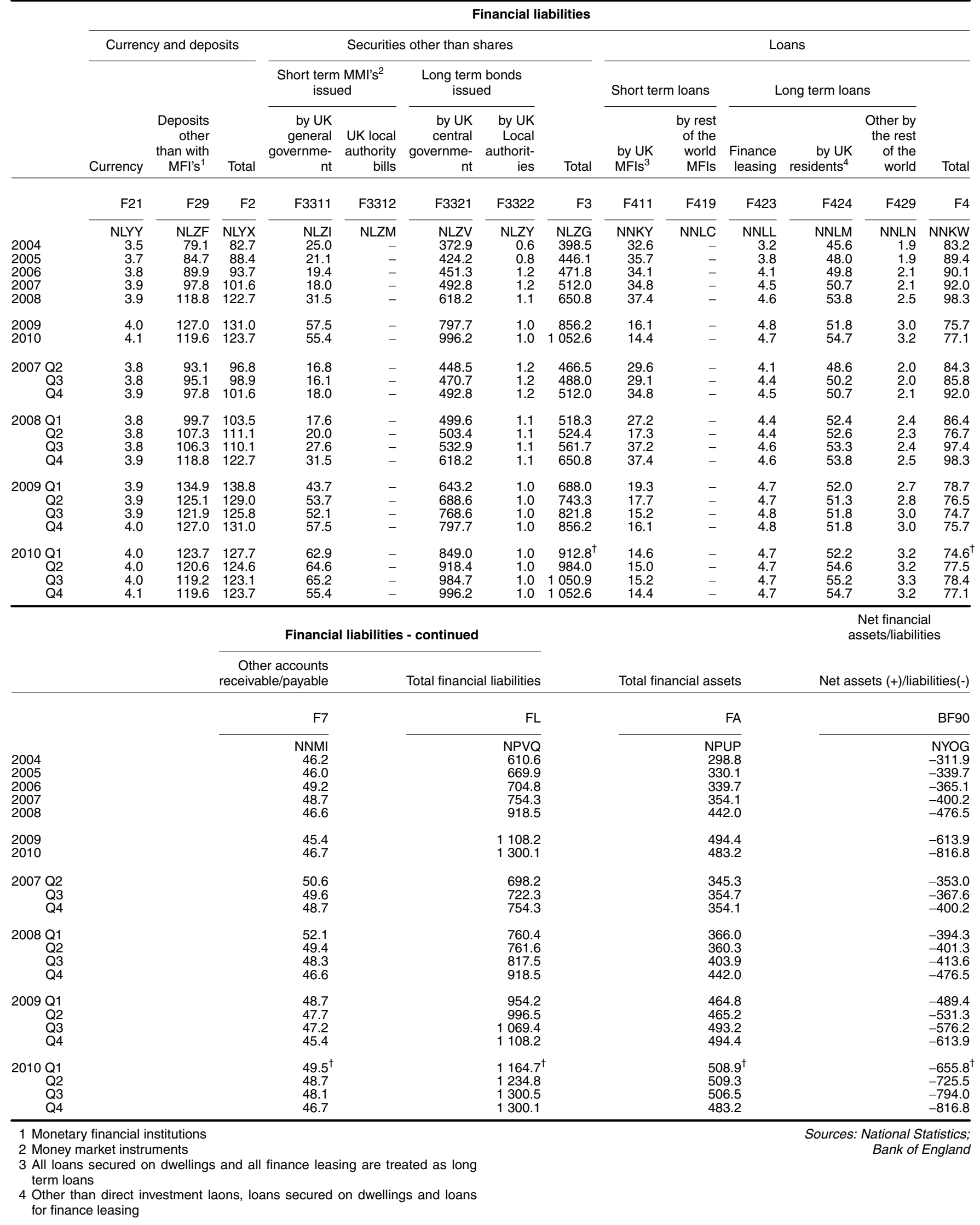




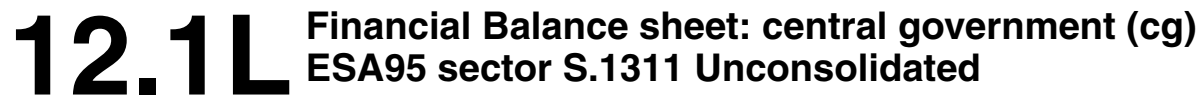

$£$ billion

\begin{tabular}{|c|c|c|c|c|c|c|c|c|c|c|c|c|c|}
\hline & \multicolumn{13}{|c|}{ Financial assets } \\
\hline & \multirow[b]{3}{*}{$\begin{array}{r}\text { Monetary } \\
\text { gold and } \\
\text { SDRs }\end{array}$} & \multicolumn{4}{|c|}{ Currency and deposits } & \multicolumn{8}{|c|}{ Securities other than shares } \\
\hline & & \multirow[b]{2}{*}{$\begin{array}{c}\text { Deposits } \\
\text { with UK } \\
\text { MFl's }^{1}\end{array}$} & \multirow[b]{2}{*}{$\begin{array}{r}\text { Deposits } \\
\text { with rest } \\
\text { of the } \\
\text { world } \\
\text { MFl's }\end{array}$} & \multirow[b]{2}{*}{$\begin{array}{l}\text { Deposits } \\
\text { other than } \\
\text { with MFI's }\end{array}$} & \multirow[b]{2}{*}{ Total } & \multicolumn{3}{|c|}{ Short term MMl's ${ }^{2}$ issued } & \multicolumn{4}{|c|}{ Bonds issued } & \multirow[b]{2}{*}{ Tota } \\
\hline & & & & & & by UK MFIs & $\begin{array}{r}\text { by other } \\
\text { UK } \\
\text { residents }\end{array}$ & $\begin{array}{l}\text { by the } \\
\text { rest of } \\
\text { the world }\end{array}$ & $\begin{array}{r}\text { by UK } \\
\text { local } \\
\text { authoriti- } \\
\text { es }\end{array}$ & $\begin{array}{r}\text { Other by } \\
\text { UK } \\
\text { residents }\end{array}$ & $\begin{array}{l}\text { by the } \\
\text { rest of } \\
\text { the world }\end{array}$ & $\begin{array}{r}\text { Financial } \\
\text { derivativ- } \\
\text { es }\end{array}$ & \\
\hline & $\mathrm{F} 1$ & $\mathrm{~F} 221$ & F229 & F29 & $\mathrm{F} 2$ & F3315 & F3316 & F3319 & F3322 & F3326 & F3329 & F34 & F3 \\
\hline $\begin{array}{l}2004 \\
2005 \\
2006 \\
2007 \\
2008\end{array}$ & $\begin{array}{r}\text { NIFC } \\
2.5 \\
3.2 \\
3.4 \\
4.3 \\
6.3\end{array}$ & $\begin{array}{r}\mathrm{NIFI} \\
5.5 \\
5.6 \\
7.7 \\
9.7 \\
28.5\end{array}$ & $\begin{array}{r}\text { NIFM } \\
3.3 \\
2.0 \\
1.2 \\
1.0 \\
4.6\end{array}$ & $\begin{array}{r}\text { NIFN } \\
- \\
- \\
- \\
3.8 \\
14.8\end{array}$ & $\begin{array}{r}\text { NIFF } \\
8.8 \\
7.6 \\
8.9 \\
14.5 \\
47.9\end{array}$ & $\begin{array}{r}\text { NSUO } \\
0.8 \\
1.0 \\
2.7 \\
0.8 \\
2.6\end{array}$ & $\begin{array}{r}\text { NSRH } \\
- \\
\overline{-} \\
1.2 \\
0.1 \\
0.1\end{array}$ & $\begin{array}{r}\text { NIGB } \\
0.3 \\
1.7 \\
3.0 \\
5.5 \\
5.3\end{array}$ & $\begin{array}{r}\text { NIGG } \\
- \\
- \\
- \\
- \\
-\end{array}$ & $\begin{array}{r}\text { NIGK } \\
0.1 \\
0.9 \\
0.3 \\
0.3 \\
5.3\end{array}$ & $\begin{array}{r}\text { NIGL } \\
17.1 \\
17.5 \\
15.3 \\
18.2 \\
24.4\end{array}$ & $\begin{array}{r}\text { ZYBQ } \\
0.2 \\
0.6 \\
0.7 \\
-0.4 \\
-3.5\end{array}$ & $\begin{array}{r}\text { NIFO } \\
18.4 \\
21.8 \\
23.3 \\
24.4 \\
34.1\end{array}$ \\
\hline $\begin{array}{l}2009 \\
2010\end{array}$ & $\begin{array}{l}15.7 \\
18.2\end{array}$ & $\begin{array}{r}35.2 \\
7.7\end{array}$ & $\begin{array}{l}4.9 \\
6.0\end{array}$ & $\begin{array}{l}21.9 \\
12.1\end{array}$ & $\begin{array}{l}61.9 \\
25.7\end{array}$ & $\begin{array}{r}0.4 \\
-\end{array}$ & $\begin{array}{l}1.0 \\
2.3\end{array}$ & $\begin{array}{l}5.6 \\
6.1\end{array}$ & - & $\begin{array}{l}0.1 \\
0.1\end{array}$ & $\begin{array}{l}21.1 \\
27.1\end{array}$ & $\begin{array}{l}-1.4 \\
-0.6\end{array}$ & $\begin{array}{l}26.8 \\
35.0\end{array}$ \\
\hline $\begin{array}{r}2007 \text { Q2 } \\
\text { Q3 } \\
\text { Q4 }\end{array}$ & $\begin{array}{l}3.4 \\
3.8 \\
4.3\end{array}$ & $\begin{array}{r}12.8 \\
16.0 \\
9.7\end{array}$ & $\begin{array}{l}0.8 \\
0.9 \\
1.0\end{array}$ & $\begin{array}{l}0.1 \\
0.3 \\
3.8\end{array}$ & $\begin{array}{l}13.7 \\
17.2 \\
14.5\end{array}$ & $\begin{array}{l}5.7 \\
2.3 \\
0.8\end{array}$ & $\begin{array}{l}0.3 \\
0.1 \\
0.1\end{array}$ & $\begin{array}{l}4.0 \\
5.5 \\
5.5\end{array}$ & $\begin{array}{l}- \\
- \\
-\end{array}$ & $\begin{array}{l}0.3 \\
0.3 \\
0.3\end{array}$ & $\begin{array}{l}14.0 \\
16.1 \\
18.2\end{array}$ & $\begin{array}{r}0.7 \\
0.2 \\
-0.4\end{array}$ & $\begin{array}{l}25.0 \\
24.4 \\
24.4\end{array}$ \\
\hline $\begin{array}{r}2008 \text { Q1 } \\
\text { Q2 } \\
\text { Q3 } \\
\text { Q4 }\end{array}$ & $\begin{array}{l}4.9 \\
4.9 \\
5.2 \\
6.3\end{array}$ & $\begin{array}{r}8.1 \\
8.3 \\
28.1 \\
28.5\end{array}$ & $\begin{array}{l}1.9 \\
2.4 \\
1.8 \\
4.6\end{array}$ & $\begin{array}{r}6.0 \\
3.7 \\
6.2 \\
14.8\end{array}$ & $\begin{array}{l}16.0 \\
14.4 \\
36.2 \\
47.9\end{array}$ & $\begin{array}{r}0.3 \\
0.2 \\
-\overline{6}\end{array}$ & $\begin{array}{l}0.1 \\
0.1 \\
0.1 \\
0.1\end{array}$ & $\begin{array}{l}8.7 \\
1.4 \\
7.1 \\
5.3\end{array}$ & $\begin{array}{l}- \\
- \\
- \\
-\end{array}$ & $\begin{array}{l}0.3 \\
0.3 \\
0.3 \\
5.3\end{array}$ & $\begin{array}{l}19.9 \\
18.8 \\
18.9 \\
24.4\end{array}$ & $\begin{array}{l}-1.3 \\
-0.9 \\
-1.1 \\
-3.5\end{array}$ & $\begin{array}{l}27.9 \\
19.8 \\
25.3 \\
34.1\end{array}$ \\
\hline $\begin{array}{r}2009 \text { Q1 } \\
\text { Q2 } \\
\text { Q3 } \\
\text { Q4 }\end{array}$ & $\begin{array}{r}6.7 \\
5.9 \\
14.9 \\
15.7\end{array}$ & $\begin{array}{l}28.8 \\
32.4 \\
35.1 \\
35.2\end{array}$ & $\begin{array}{l}4.3 \\
4.9 \\
5.4 \\
4.9\end{array}$ & $\begin{array}{l}30.6 \\
20.2 \\
39.6 \\
21.9\end{array}$ & $\begin{array}{l}63.7 \\
57.6 \\
80.0 \\
61.9\end{array}$ & $\begin{array}{l}2.3 \\
2.5 \\
2.4 \\
0.4\end{array}$ & $\begin{array}{l}0.2 \\
1.2 \\
1.1 \\
1.0\end{array}$ & $\begin{array}{l}5.8 \\
9.6 \\
7.0 \\
5.6\end{array}$ & $\begin{array}{l}- \\
- \\
- \\
-\end{array}$ & $\begin{array}{l}9.1 \\
0.1 \\
0.1 \\
0.1\end{array}$ & $\begin{array}{l}23.7 \\
21.3 \\
21.8 \\
21.1\end{array}$ & $\begin{array}{l}-2.8 \\
-1.4 \\
-1.6 \\
-1.4\end{array}$ & $\begin{array}{l}38.3 \\
33.2 \\
30.7 \\
26.8\end{array}$ \\
\hline $\begin{array}{r}2010 \text { Q1 } \\
\text { Q2 } \\
\text { Q3 } \\
\text { Q4 }\end{array}$ & $\begin{array}{l}16.5 \\
17.3 \\
17.3 \\
18.2\end{array}$ & $\begin{array}{r}22.0 \\
17.0 \\
8.4 \\
7.7\end{array}$ & $\begin{array}{l}6.4 \\
6.2 \\
6.9 \\
6.0\end{array}$ & $\begin{array}{l}37.0 \\
25.1 \\
31.3 \\
12.1\end{array}$ & $\begin{array}{l}65.5 \\
48.2 \\
46.7 \\
25.7\end{array}$ & $\begin{array}{l}- \\
- \\
- \\
-\end{array}$ & $\begin{array}{l}0.6 \\
1.7 \\
1.7 \\
2.3\end{array}$ & $\begin{array}{r}4.0 \\
7.8 \\
11.4 \\
6.1\end{array}$ & $\begin{array}{l}- \\
- \\
- \\
-\end{array}$ & $\begin{array}{l}0.1 \\
0.1 \\
0.1 \\
0.1\end{array}$ & $\begin{array}{l}22.4 \\
24.0 \\
25.3 \\
27.1\end{array}$ & $\begin{array}{l}-1.0 \\
-0.5 \\
-0.6 \\
-0.6\end{array}$ & $\begin{array}{l}26.0 \\
33.2 \\
37.8 \\
35.0\end{array}$ \\
\hline
\end{tabular}

\begin{tabular}{|c|c|c|c|c|c|c|c|c|c|c|c|}
\hline & \multicolumn{11}{|c|}{ Financial assets - continued } \\
\hline & \multicolumn{3}{|c|}{ Loans } & \multicolumn{6}{|c|}{ Shares and other equity } & \multirow{3}{*}{$\begin{array}{r}\text { Other } \\
\text { accounts } \\
\text { receivable/- } \\
\text { payable }\end{array}$} & \multirow{3}{*}{$\begin{array}{r}\text { Total } \\
\text { financial } \\
\text { assets }\end{array}$} \\
\hline & \multicolumn{2}{|c|}{ Long term loans } & \multirow[b]{2}{*}{ Total } & \multicolumn{5}{|c|}{ Excl mutual funds' shares } & \multirow[b]{2}{*}{ Total } & & \\
\hline & $\begin{array}{l}\text { Secured on } \\
\text { dwellings }\end{array}$ & $\begin{array}{r}\text { by UK } \\
\text { residents }^{3}\end{array}$ & & $\begin{array}{r}\text { Quoted UK } \\
\text { shares }\end{array}$ & $\begin{array}{r}\text { Other UK } \\
\text { equity }\end{array}$ & $\begin{array}{r}\text { Unquoted UK } \\
\text { shares }\end{array}$ & $\begin{array}{r}\text { by UK } \\
\text { residents }\end{array}$ & $\begin{array}{l}\text { by the rest } \\
\text { of the world }\end{array}$ & & & \\
\hline & $\mathrm{F} 422$ & $\mathrm{~F} 424$ & $\mathrm{~F} 4$ & F514 & F515 & F516 & F517 & F519 & F5 & F7 & $\mathrm{FA}$ \\
\hline $\begin{array}{l}2004 \\
2005 \\
2006 \\
2007 \\
2008\end{array}$ & $\begin{array}{r}\text { NIHB } \\
0.1 \\
0.1 \\
0.1 \\
0.1 \\
0.1\end{array}$ & $\begin{array}{r}\text { NIHG } \\
69.4 \\
74.3 \\
77.7 \\
83.3 \\
89.2\end{array}$ & $\begin{array}{r}\text { NIGQ } \\
69.4 \\
74.4 \\
77.8 \\
83.4 \\
89.3\end{array}$ & $\begin{array}{r}\text { NIHN } \\
-\overline{3} \\
0.6 \\
0.6 \\
0.5 \\
12.8\end{array}$ & $\begin{array}{r}\mathrm{H} 4 \mathrm{O} 7 \\
7.1 \\
12.7 \\
9.8 \\
7.8 \\
8.9\end{array}$ & $\begin{array}{r}\mathrm{NIHO} \\
1.4 \\
1.4 \\
3.9 \\
2.3 \\
5.9\end{array}$ & $\begin{array}{r}\text { NSNX } \\
- \\
- \\
- \\
- \\
-\end{array}$ & $\begin{array}{r}\text { NIHS } \\
8.0 \\
8.7 \\
9.5 \\
10.2 \\
11.2\end{array}$ & $\begin{array}{l}\mathrm{NIHI} \\
16.5 \\
23.1 \\
23.8 \\
20.8 \\
38.8\end{array}$ & $\begin{array}{l}\text { NIIC } \\
47.2 \\
52.5 \\
55.1 \\
54.0 \\
70.4\end{array}$ & $\begin{array}{l}\text { NIGP } \\
162.8 \\
182.4 \\
192.3 \\
201.6 \\
286.8\end{array}$ \\
\hline $\begin{array}{l}2009 \\
2010\end{array}$ & $\begin{array}{l}0.1 \\
0.1\end{array}$ & $\begin{array}{r}92.1 \\
100.6\end{array}$ & $\begin{array}{r}92.2 \\
100.7\end{array}$ & $\begin{array}{l}54.1 \\
54.1\end{array}$ & $\begin{array}{l}3.8 \\
2.8\end{array}$ & $\begin{array}{l}5.9 \\
5.8\end{array}$ & - & $\begin{array}{l}12.3 \\
13.5\end{array}$ & $\begin{array}{l}76.0 \\
76.3\end{array}$ & $\begin{array}{l}72.4 \\
75.4\end{array}$ & $\begin{array}{l}345.0 \\
331.3\end{array}$ \\
\hline $\begin{array}{r}2007 \text { Q2 } \\
\text { Q3 } \\
\text { Q4 }\end{array}$ & $\begin{array}{l}0.1 \\
0.1 \\
0.1\end{array}$ & $\begin{array}{l}78.0 \\
81.7 \\
83.3\end{array}$ & $\begin{array}{l}78.1 \\
81.7 \\
83.4\end{array}$ & $\begin{array}{l}0.6 \\
0.5 \\
0.5\end{array}$ & $\begin{array}{l}6.6 \\
7.9 \\
7.8\end{array}$ & $\begin{array}{l}2.6 \\
2.3 \\
2.3\end{array}$ & $\begin{array}{l}- \\
- \\
-\end{array}$ & $\begin{array}{l}10.2 \\
10.2 \\
10.2\end{array}$ & $\begin{array}{l}20.0 \\
21.0 \\
20.8\end{array}$ & $\begin{array}{l}55.2 \\
53.9 \\
54.0\end{array}$ & $\begin{array}{l}195.4 \\
202.1 \\
201.6\end{array}$ \\
\hline $\begin{array}{r}2008 \text { Q1 } \\
\text { Q2 } \\
\text { Q3 } \\
\text { Q4 }\end{array}$ & $\begin{array}{l}0.1 \\
0.1 \\
0.1 \\
0.1\end{array}$ & $\begin{array}{l}85.0 \\
86.2 \\
87.6 \\
89.2\end{array}$ & $\begin{array}{l}85.1 \\
86.3 \\
87.7 \\
89.3\end{array}$ & $\begin{array}{r}0.5 \\
0.6 \\
0.3 \\
12.8\end{array}$ & $\begin{array}{l}7.6 \\
7.6 \\
8.3 \\
8.9\end{array}$ & $\begin{array}{l}2.3 \\
2.4 \\
5.8 \\
5.9\end{array}$ & $\begin{array}{l}- \\
- \\
- \\
-\end{array}$ & $\begin{array}{l}10.3 \\
10.8 \\
11.0 \\
11.2\end{array}$ & $\begin{array}{l}20.7 \\
21.3 \\
25.4 \\
38.8\end{array}$ & $\begin{array}{l}55.7 \\
54.3 \\
65.3 \\
70.4\end{array}$ & $\begin{array}{l}210.2 \\
201.0 \\
245.1 \\
286.8\end{array}$ \\
\hline $\begin{array}{r}2009 \text { Q1 } \\
\text { Q2 } \\
\text { Q3 } \\
\text { Q4 }\end{array}$ & $\begin{array}{l}0.1 \\
0.1 \\
0.1 \\
0.1\end{array}$ & $\begin{array}{l}89.4 \\
90.5 \\
91.5 \\
92.1\end{array}$ & $\begin{array}{l}89.5 \\
90.6 \\
91.6 \\
92.2\end{array}$ & $\begin{array}{l}22.2 \\
28.9 \\
28.9 \\
54.1\end{array}$ & $\begin{array}{l}4.6 \\
4.0 \\
3.8 \\
3.8\end{array}$ & $\begin{array}{l}6.0 \\
6.0 \\
5.9 \\
5.9\end{array}$ & $\begin{array}{l}- \\
- \\
- \\
-\end{array}$ & $\begin{array}{l}11.2 \\
12.0 \\
12.1 \\
12.3\end{array}$ & $\begin{array}{l}44.0 \\
50.9 \\
50.7 \\
76.0\end{array}$ & $\begin{array}{l}69.6 \\
72.5 \\
71.5 \\
72.4\end{array}$ & $\begin{array}{l}311.7 \\
310.8 \\
339.4 \\
345.0\end{array}$ \\
\hline $\begin{array}{r}2010 \text { Q1 } \\
\text { Q2 } \\
\text { Q3 } \\
\text { Q4 }\end{array}$ & $\begin{array}{l}0.1 \\
0.1 \\
0.1 \\
0.1\end{array}$ & $\begin{array}{r}94.0 \\
98.7 \\
99.9 \\
100.6\end{array}$ & $\begin{array}{r}94.1 \\
98.8 \\
100.0 \\
100.7\end{array}$ & $\begin{array}{l}54.1 \\
54.1 \\
54.1 \\
54.1\end{array}$ & $\begin{array}{l}3.3^{\dagger} \\
2.9 \\
2.6 \\
2.8\end{array}$ & $\begin{array}{l}5.8 \\
5.8 \\
5.8 \\
5.8\end{array}$ & $\begin{array}{l}- \\
- \\
- \\
-\end{array}$ & $\begin{array}{l}12.3 \\
12.4 \\
13.1 \\
13.5\end{array}$ & $\begin{array}{l}75.6 \\
75.2 \\
75.6 \\
76.3\end{array}$ & $\begin{array}{l}79.6^{\dagger} \\
80.2 \\
73.7 \\
75.4\end{array}$ & $\begin{array}{l}357.3^{\dagger} \\
353.0 \\
351.0 \\
331.3\end{array}$ \\
\hline $\begin{array}{l}1 \text { Monet } \\
2 \text { Money } \\
3 \text { Other } \\
\text { for fina }\end{array}$ & $\begin{array}{l}\text { ancial instituti } \\
\text { et instruments } \\
\text { lirect investm } \\
\text { asing. }\end{array}$ & loans, loar & . & dwellings & loans & & & & & $\begin{array}{r}\text { Irces: National } \\
\text { Bank }\end{array}$ & $\begin{array}{l}\text { Statistics; } \\
\text { f England }\end{array}$ \\
\hline
\end{tabular}




\begin{tabular}{|c|c|c|c|c|c|c|c|c|c|c|c|c|c|c|}
\hline & \multicolumn{2}{|c|}{$\begin{array}{l}\text { Currency and } \\
\text { deposits }\end{array}$} & \multirow[b]{3}{*}{ Total } & \multicolumn{5}{|c|}{ Securities other than shares } & \multicolumn{6}{|c|}{ Loans } \\
\hline & \multirow[b]{2}{*}{ Currency } & \multirow[b]{2}{*}{$\begin{array}{r}\text { Deposits } \\
\text { other } \\
\text { than with } \\
\text { MFI's }^{1}\end{array}$} & & $\begin{array}{r}\text { Short term } \\
\text { issu }\end{array}$ & $\begin{array}{l}\text { MMl's }{ }^{2} \\
\text { ed }\end{array}$ & $\begin{array}{r}\text { Long terr } \\
\text { issu }\end{array}$ & $\begin{array}{l}n \text { bonds } \\
\text { led }\end{array}$ & \multirow[b]{2}{*}{ Total } & \multicolumn{2}{|c|}{ Short term loans } & \multicolumn{3}{|c|}{ Long term loans } & \multirow[b]{2}{*}{ Total } \\
\hline & & & & $\begin{array}{r}\text { Sterling } \\
\text { Treasury } \\
\text { Bills }\end{array}$ & $\begin{array}{r}\text { ECU } \\
\text { Treasury } \\
\text { Bills }\end{array}$ & $\begin{array}{r}\text { British } \\
\text { governme- } \\
\mathrm{nt} \\
\text { securiti- } \\
\text { es }\end{array}$ & $\begin{array}{r}\text { Other } \\
\text { central } \\
\text { governme- } \\
n t \\
\text { sterling } \\
\text { bonds }\end{array}$ & & $\begin{array}{l}\text { by UK } \\
\text { MFIs }^{3}\end{array}$ & $\begin{array}{c}\text { by rest } \\
\text { of the } \\
\text { world } \\
\text { MFIs }\end{array}$ & $\begin{array}{r}\text { Finance } \\
\text { leasing }\end{array}$ & $\begin{array}{r}\text { by UK } \\
\text { residents }{ }^{4}\end{array}$ & $\begin{array}{r}\text { Other by } \\
\text { the rest } \\
\text { of the } \\
\text { world }\end{array}$ & \\
\hline & F21 & $\mathrm{F} 29$ & $\mathrm{~F} 2$ & F33111 & F33112 & F33211 & F33212 & F3 & F411 & F419 & F423 & $\mathrm{F} 424$ & F429 & F4 \\
\hline $\begin{array}{l}2004 \\
2005 \\
2006 \\
2007 \\
2008\end{array}$ & $\begin{array}{r}\text { NIIK } \\
3.5 \\
3.7 \\
3.8 \\
3.9 \\
3.9\end{array}$ & $\begin{array}{r}\text { NIIR } \\
79.1 \\
84.7 \\
89.9 \\
97.8 \\
118.8\end{array}$ & $\begin{array}{r}\mathrm{NIIJ} \\
82.7 \\
88.4 \\
93.7 \\
101.6 \\
122.7\end{array}$ & $\begin{array}{l}\text { NIIV } \\
25.0 \\
21.1 \\
19.4 \\
18.0 \\
31.5\end{array}$ & $\begin{array}{r}\text { NIIW } \\
- \\
- \\
- \\
- \\
-\end{array}$ & $\begin{array}{r}\text { NIJI } \\
370.1 \\
421.3 \\
448.4 \\
490.0 \\
616.9\end{array}$ & $\begin{array}{r}\text { NIJJ } \\
2.8 \\
3.0 \\
2.9 \\
2.8 \\
1.4\end{array}$ & $\begin{array}{r}\text { NIIS } \\
397.9 \\
445.3 \\
470.6 \\
510.8 \\
649.7\end{array}$ & $\begin{array}{r}\text { NIJW } \\
26.3 \\
28.1 \\
25.0 \\
24.5 \\
26.5\end{array}$ & $\begin{array}{r}\text { NIKA } \\
- \\
- \\
- \\
- \\
-\end{array}$ & $\begin{array}{r}\text { NIKJ } \\
3.2 \\
3.7 \\
4.0 \\
4.4 \\
4.5\end{array}$ & $\begin{array}{r}\text { NIKK } \\
0.1 \\
0.1 \\
0.1 \\
- \\
-\end{array}$ & $\begin{array}{r}\text { NIKL } \\
0.1 \\
0.1 \\
- \\
- \\
-\end{array}$ & $\begin{array}{l}\text { NIJU } \\
29.8 \\
31.9 \\
29.1 \\
29.0 \\
31.0\end{array}$ \\
\hline $\begin{array}{l}2009 \\
2010\end{array}$ & $\begin{array}{l}4.0 \\
4.1\end{array}$ & $\begin{array}{l}127.0 \\
119.6\end{array}$ & $\begin{array}{l}131.0 \\
123.7\end{array}$ & $\begin{array}{l}57.5 \\
55.4\end{array}$ & $\begin{array}{l}- \\
-\end{array}$ & $\begin{array}{l}796.3 \\
994.9\end{array}$ & $\begin{array}{l}1.3 \\
1.4\end{array}$ & $\begin{array}{r}855.1 \\
1051.6\end{array}$ & $\begin{array}{l}5.0 \\
2.8\end{array}$ & $\begin{array}{l}- \\
-\end{array}$ & $\begin{array}{l}4.7 \\
4.7\end{array}$ & $\begin{array}{l}- \\
-\end{array}$ & $\begin{array}{l}- \\
-\end{array}$ & $\begin{array}{l}9.8 \\
7.4\end{array}$ \\
\hline $\begin{array}{r}2007 \text { Q2 } \\
\text { Q3 } \\
\text { Q4 }\end{array}$ & $\begin{array}{l}3.8 \\
3.8 \\
3.9\end{array}$ & $\begin{array}{l}93.1 \\
95.1 \\
97.8\end{array}$ & $\begin{array}{r}96.8 \\
98.9 \\
101.6\end{array}$ & $\begin{array}{l}16.8 \\
16.1 \\
18.0\end{array}$ & $\begin{array}{l}- \\
- \\
-\end{array}$ & $\begin{array}{l}445.7 \\
467.9 \\
490.0\end{array}$ & $\begin{array}{l}2.8 \\
2.8 \\
2.8\end{array}$ & $\begin{array}{l}465.3 \\
486.8 \\
510.8\end{array}$ & $\begin{array}{l}20.3 \\
19.4 \\
24.5\end{array}$ & $\begin{array}{l}- \\
- \\
-\end{array}$ & $\begin{array}{l}4.1 \\
4.4 \\
4.4\end{array}$ & $\begin{array}{l}- \\
- \\
-\end{array}$ & $\begin{array}{l}- \\
- \\
-\end{array}$ & $\begin{array}{l}24.4 \\
23.8 \\
29.0\end{array}$ \\
\hline $\begin{array}{r}2008 \text { Q1 } \\
\text { Q2 } \\
\text { Q3 } \\
\text { Q4 }\end{array}$ & $\begin{array}{l}3.8 \\
3.8 \\
3.8 \\
3.9\end{array}$ & $\begin{array}{r}99.7 \\
107.3 \\
106.3 \\
118.8\end{array}$ & $\begin{array}{l}103.5 \\
111.1 \\
110.1 \\
122.7\end{array}$ & $\begin{array}{l}17.6 \\
20.0 \\
27.6 \\
31.5\end{array}$ & $\begin{array}{l}- \\
- \\
- \\
-\end{array}$ & $\begin{array}{l}496.7 \\
500.5 \\
531.6 \\
616.9\end{array}$ & $\begin{array}{l}2.9 \\
2.9 \\
1.4 \\
1.4\end{array}$ & $\begin{array}{l}517.2 \\
523.3 \\
560.5 \\
649.7\end{array}$ & $\begin{array}{r}16.5 \\
6.5 \\
26.1 \\
26.5\end{array}$ & $\begin{array}{l}- \\
- \\
- \\
-\end{array}$ & $\begin{array}{l}4.3 \\
4.4 \\
4.5 \\
4.5\end{array}$ & $\begin{array}{l}- \\
- \\
- \\
-\end{array}$ & $\begin{array}{l}- \\
- \\
- \\
-\end{array}$ & $\begin{array}{l}20.8 \\
10.9 \\
30.6 \\
31.0\end{array}$ \\
\hline $\begin{array}{r}2009 \text { Q1 } \\
\text { Q2 } \\
\text { Q3 } \\
\text { Q4 }\end{array}$ & $\begin{array}{l}3.9 \\
3.9 \\
3.9 \\
4.0\end{array}$ & $\begin{array}{l}134.9 \\
125.1 \\
121.9 \\
127.0\end{array}$ & $\begin{array}{l}138.8 \\
129.0 \\
125.8 \\
131.0\end{array}$ & $\begin{array}{l}43.7 \\
53.7 \\
52.1 \\
57.5\end{array}$ & $\begin{array}{l}- \\
- \\
- \\
-\end{array}$ & $\begin{array}{l}641.8 \\
687.2 \\
767.2 \\
796.3\end{array}$ & $\begin{array}{l}1.4 \\
1.4 \\
1.4 \\
1.3\end{array}$ & $\begin{array}{l}686.9 \\
742.3 \\
820.7 \\
855.1\end{array}$ & $\begin{array}{l}7.9 \\
6.9 \\
4.2 \\
5.0\end{array}$ & $\begin{array}{l}- \\
- \\
- \\
-\end{array}$ & $\begin{array}{l}4.6 \\
4.7 \\
4.7 \\
4.7\end{array}$ & $\begin{array}{l}- \\
- \\
- \\
-\end{array}$ & $\begin{array}{l}- \\
- \\
- \\
-\end{array}$ & $\begin{array}{r}12.5 \\
11.6 \\
8.9 \\
9.8\end{array}$ \\
\hline \multirow[t]{2}{*}{$\begin{array}{r}2010 \text { Q1 } \\
\text { Q2 } \\
\text { Q3 } \\
\text { Q4 }\end{array}$} & $\begin{array}{l}4.0 \\
4.0 \\
4.0 \\
4.1\end{array}$ & $\begin{array}{l}123.7 \\
120.6 \\
119.2 \\
119.6\end{array}$ & $\begin{array}{l}127.7 \\
124.6 \\
123.1 \\
123.7\end{array}$ & $\begin{array}{l}62.9 \\
64.6 \\
65.2 \\
55.4\end{array}$ & $\begin{array}{l}- \\
- \\
- \\
-\end{array}$ & $\begin{array}{l}847.6 \\
917.0 \\
983.3 \\
994.9\end{array}$ & $\begin{array}{l}1.4 \\
1.4 \\
1.4 \\
1.4\end{array}$ & $\begin{array}{r}911.8 \\
983.0 \\
1049.9 \\
1051.6\end{array}$ & $\begin{array}{l}3.0 \\
3.4 \\
3.8 \\
2.8\end{array}$ & $\begin{array}{l}- \\
- \\
- \\
-\end{array}$ & $\begin{array}{l}4.6 \\
4.6^{\dagger} \\
4.7 \\
4.7\end{array}$ & $\begin{array}{l}- \\
- \\
- \\
-\end{array}$ & $\begin{array}{l}- \\
- \\
- \\
-\end{array}$ & $\begin{array}{l}7.6 \\
8.0^{\dagger} \\
8.5 \\
7.4\end{array}$ \\
\hline & & & $\begin{array}{l}\text { Oth } \\
\text { receiva }\end{array}$ & $\begin{array}{l}\text { accounts } \\
\text { /payable }\end{array}$ & & Total financi & al liabilities & & Total fina & cial assets & & Net ass & ts $(+) /$ /iabilit & ities(-) \\
\hline
\end{tabular}

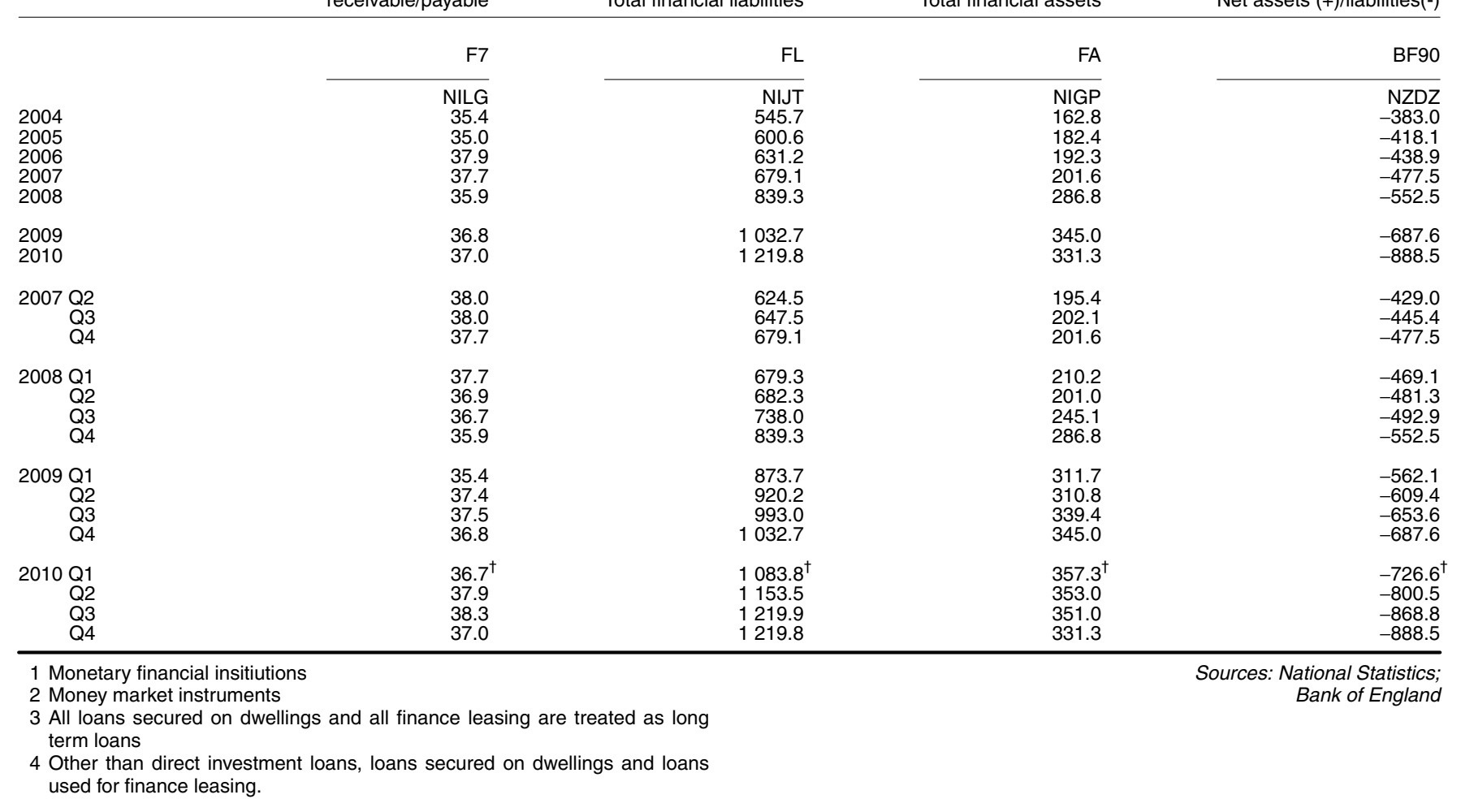




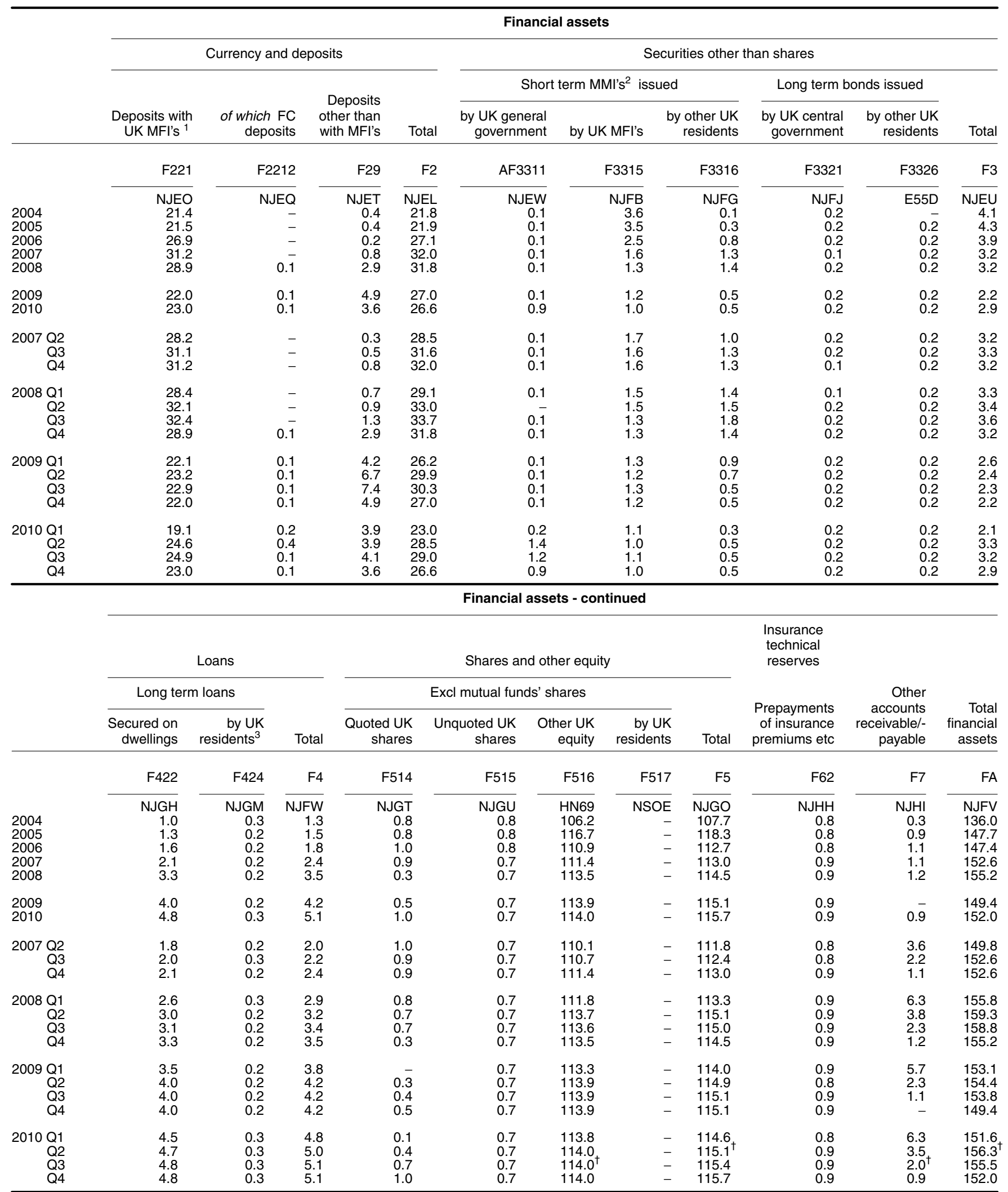

1 Monetary financial institutions

2 Money market instruments

Sources: National Statistics;

3 Other than direct investment loans, loans secured on dwellings and loans

Bank of England

used for finance leasing 


\begin{tabular}{|c|c|c|c|c|c|c|c|c|c|}
\hline & \multicolumn{9}{|c|}{ Financial liabilities } \\
\hline & \multicolumn{3}{|c|}{ Securities other than shares } & \multicolumn{5}{|c|}{ Loans } & \multirow[b]{3}{*}{ Total } \\
\hline & $\begin{array}{l}\text { Short term } \\
\text { MMl's }^{1} \text { issued }\end{array}$ & $\begin{array}{l}\text { Long term bonds } \\
\text { issued }\end{array}$ & & \multicolumn{2}{|c|}{ Short term loans } & \multicolumn{3}{|c|}{ Long term loans } & \\
\hline & $\begin{array}{r}\text { by Local } \\
\text { authorities }\end{array}$ & $\begin{array}{r}\text { by UK Local } \\
\text { authorities }\end{array}$ & Total & by UK MFIs ${ }^{2,3}$ & $\begin{array}{l}\text { by rest of the } \\
\text { world MFIs }\end{array}$ & \multicolumn{2}{|r|}{ by UK residents ${ }^{4}$} & $\begin{array}{r}\text { Other by the } \\
\text { rest of the } \\
\text { world }\end{array}$ & \\
\hline & F3312 & F3322 & F3 & F411 & F419 & F423 & F424 & F429 & $\mathrm{F} 4$ \\
\hline $\begin{array}{l}2004 \\
2005 \\
2006 \\
2007 \\
2008\end{array}$ & $\begin{array}{r}\text { NJIE } \\
- \\
- \\
- \\
- \\
-\end{array}$ & $\begin{array}{r}\text { NJIQ } \\
0.6 \\
0.8 \\
1.2 \\
1.2 \\
1.1\end{array}$ & $\begin{array}{r}\text { NJHY } \\
0.6 \\
0.8 \\
1.2 \\
1.2 \\
1.1\end{array}$ & $\begin{array}{r}\text { NJJC } \\
6.3 \\
7.6 \\
9.1 \\
10.3 \\
10.9\end{array}$ & $\begin{array}{r}\text { NJJG } \\
- \\
- \\
- \\
- \\
-\end{array}$ & $\begin{array}{r}\text { NJJP } \\
-\overline{-} \\
0.1 \\
0.1 \\
0.1 \\
0.1\end{array}$ & $\begin{array}{r}\text { NJJQ } \\
45.5 \\
48.0 \\
49.8 \\
50.6 \\
53.8\end{array}$ & $\begin{array}{r}\text { NJJR } \\
1.7 \\
1.9 \\
2.1 \\
2.1 \\
2.5\end{array}$ & $\begin{array}{r}\text { NJJA } \\
53.5 \\
57.5 \\
61.1 \\
63.1 \\
67.3\end{array}$ \\
\hline $\begin{array}{l}2009 \\
2010\end{array}$ & $\begin{array}{l}- \\
-\end{array}$ & $\begin{array}{l}1.0 \\
1.0\end{array}$ & $\begin{array}{l}1.0 \\
1.0\end{array}$ & $\begin{array}{l}11.0 \\
11.7\end{array}$ & $\begin{array}{l}- \\
-\end{array}$ & $\begin{array}{l}0.1 \\
0.1\end{array}$ & $\begin{array}{l}51.8 \\
54.7\end{array}$ & $\begin{array}{l}3.0 \\
3.2\end{array}$ & $\begin{array}{l}65.9 \\
69.6\end{array}$ \\
\hline $\begin{array}{r}2007 \text { Q2 } \\
\text { Q3 } \\
\text { Q4 }\end{array}$ & $\begin{array}{l}- \\
- \\
-\end{array}$ & $\begin{array}{l}1.2 \\
1.2 \\
1.2\end{array}$ & $\begin{array}{l}1.2 \\
1.2 \\
1.2\end{array}$ & $\begin{array}{r}9.3 \\
9.7 \\
10.3\end{array}$ & $\begin{array}{l}- \\
- \\
-\end{array}$ & $\begin{array}{l}0.1 \\
0.1 \\
0.1\end{array}$ & $\begin{array}{l}48.6 \\
50.2 \\
50.6\end{array}$ & $\begin{array}{l}2.0 \\
2.0 \\
2.1\end{array}$ & $\begin{array}{l}60.0 \\
62.0 \\
63.1\end{array}$ \\
\hline $\begin{array}{r}2008 \text { Q1 } \\
\text { Q2 } \\
\text { Q3 } \\
\text { Q4 }\end{array}$ & $\begin{array}{l}- \\
- \\
- \\
-\end{array}$ & $\begin{array}{l}1.1 \\
1.1 \\
1.1 \\
1.1\end{array}$ & $\begin{array}{l}1.1 \\
1.1 \\
1.1 \\
1.1\end{array}$ & $\begin{array}{l}10.8 \\
10.7 \\
11.0 \\
10.9\end{array}$ & $\begin{array}{l}- \\
- \\
- \\
-\end{array}$ & $\begin{array}{l}0.1 \\
0.1 \\
0.1 \\
0.1\end{array}$ & $\begin{array}{l}52.4 \\
52.6 \\
53.3 \\
53.8\end{array}$ & $\begin{array}{l}2.4 \\
2.3 \\
2.4 \\
2.5\end{array}$ & $\begin{array}{l}65.6 \\
65.7 \\
66.8 \\
67.3\end{array}$ \\
\hline $\begin{array}{r}2009 \text { Q1 } \\
\text { Q2 } \\
\text { Q3 } \\
\text { Q4 }\end{array}$ & $\begin{array}{l}- \\
- \\
- \\
-\end{array}$ & $\begin{array}{l}1.0 \\
1.0 \\
1.0 \\
1.0\end{array}$ & $\begin{array}{l}1.0 \\
1.0 \\
1.0 \\
1.0\end{array}$ & $\begin{array}{l}11.4 \\
10.9 \\
11.0 \\
11.0\end{array}$ & $\begin{array}{l}- \\
- \\
- \\
-\end{array}$ & $\begin{array}{l}0.1 \\
0.1 \\
0.1 \\
0.1\end{array}$ & $\begin{array}{l}52.0 \\
51.2 \\
51.7 \\
51.8\end{array}$ & $\begin{array}{l}2.7 \\
2.8 \\
3.0 \\
3.0\end{array}$ & $\begin{array}{l}66.2 \\
64.9 \\
65.7 \\
65.9\end{array}$ \\
\hline \multirow[t]{2}{*}{$\begin{array}{r}2010 \text { Q1 } \\
\text { Q2 } \\
\text { Q3 } \\
\text { Q4 }\end{array}$} & $\begin{array}{l}- \\
- \\
- \\
-\end{array}$ & $\begin{array}{l}1.0 \\
1.0 \\
1.0 \\
1.0\end{array}$ & $\begin{array}{l}1.0 \\
1.0 \\
1.0 \\
1.0\end{array}$ & $\begin{array}{l}11.6 \\
11.6 \\
11.4 \\
11.7\end{array}$ & $\begin{array}{l}- \\
- \\
- \\
-\end{array}$ & $\begin{array}{l}0.1 \\
0.1 \\
0.1 \\
0.1\end{array}$ & $\begin{array}{l}52.2 \\
54.5 \\
55.2 \\
54.7\end{array}$ & $\begin{array}{l}3.2 \\
3.2 \\
3.3 \\
3.2\end{array}$ & $\begin{array}{l}67.0 \\
69.4 \\
69.9 \\
69.6\end{array}$ \\
\hline & & \multicolumn{4}{|c|}{ Financial liabilities - continued } & \multicolumn{4}{|c|}{$\begin{array}{c}\text { Net financial } \\
\text { assets/liabilities } \\
\text { Net assets (+)/liabilities(-) }\end{array}$} \\
\hline & & \multicolumn{2}{|c|}{$\mathrm{F} 7$} & \multicolumn{2}{|r|}{$\mathrm{FL}$} & \multicolumn{2}{|r|}{ FA } & \multicolumn{2}{|r|}{ BF90 } \\
\hline $\begin{array}{l}2004 \\
2005 \\
2006 \\
2007 \\
2008\end{array}$ & & \multicolumn{2}{|c|}{$\begin{array}{r}\text { NJKM } \\
10.8 \\
11.0 \\
11.3 \\
11.0 \\
10.8\end{array}$} & \multicolumn{2}{|r|}{$\begin{array}{l}\text { NJIZ } \\
64.9 \\
69.3 \\
73.6 \\
75.2 \\
79.2\end{array}$} & \multicolumn{2}{|r|}{$\begin{array}{l}\text { NJFV } \\
136.0 \\
147.7 \\
147.4 \\
152.6 \\
155.2\end{array}$} & \multicolumn{2}{|r|}{$\begin{array}{r}\text { NYOJ } \\
71.1 \\
78.4 \\
73.8 \\
77.3 \\
76.0\end{array}$} \\
\hline $\begin{array}{l}2009 \\
2010\end{array}$ & & \multicolumn{2}{|c|}{$\begin{array}{l}8.7 \\
9.7\end{array}$} & \multicolumn{2}{|r|}{$\begin{array}{l}75.6 \\
80.3\end{array}$} & \multicolumn{2}{|r|}{$\begin{array}{l}149.4 \\
152.0\end{array}$} & & $\begin{array}{l}73.8 \\
71.7\end{array}$ \\
\hline $\begin{array}{r}2007 \text { Q2 } \\
\text { Q3 } \\
\text { Q4 }\end{array}$ & & \multicolumn{2}{|c|}{$\begin{array}{l}12.6 \\
11.6 \\
11.0\end{array}$} & \multicolumn{2}{|r|}{$\begin{array}{l}73.8 \\
74.8 \\
75.2\end{array}$} & \multicolumn{2}{|r|}{$\begin{array}{l}149.8 \\
152.6 \\
152.6\end{array}$} & & $\begin{array}{l}76.1 \\
77.9 \\
77.3\end{array}$ \\
\hline $\begin{array}{r}2008 \text { Q1 } \\
\text { Q2 } \\
\text { Q3 } \\
\text { Q4 }\end{array}$ & & \multicolumn{2}{|c|}{$\begin{array}{l}14.4 \\
12.5 \\
11.6 \\
10.8\end{array}$} & \multicolumn{2}{|r|}{$\begin{array}{l}81.1 \\
79.3 \\
79.5 \\
79.2\end{array}$} & \multicolumn{2}{|r|}{$\begin{array}{l}155.8 \\
159.3 \\
158.8 \\
155.2\end{array}$} & & $\begin{array}{l}74.7 \\
80.0 \\
79.3 \\
76.0\end{array}$ \\
\hline $\begin{array}{r}2009 \text { Q1 } \\
\text { Q2 } \\
\text { Q3 } \\
\text { Q4 }\end{array}$ & & \multicolumn{2}{|c|}{$\begin{array}{r}13.3 \\
10.3 \\
9.6 \\
8.7\end{array}$} & \multicolumn{2}{|r|}{$\begin{array}{l}80.5 \\
76.3 \\
76.4 \\
75.6\end{array}$} & \multicolumn{2}{|r|}{$\begin{array}{l}153.1 \\
154.4 \\
153.8 \\
149.4\end{array}$} & & $\begin{array}{l}72.7 \\
78.1 \\
77.4 \\
73.8\end{array}$ \\
\hline $\begin{array}{r}2010 \text { Q1 } \\
\text { Q2 } \\
\text { Q3 } \\
\text { Q4 }\end{array}$ & & \multicolumn{2}{|c|}{$\begin{array}{c}12.8^{\dagger} \\
10.9 \\
9.8 \\
9.7\end{array}$} & \multicolumn{2}{|r|}{$\begin{array}{l}80.8^{\dagger} \\
81.3 \\
80.7 \\
80.3\end{array}$} & \multicolumn{2}{|r|}{$\begin{array}{l}151.6^{\dagger} \\
156.3^{\dagger} \\
155.5 \\
152.0\end{array}$} & & $\begin{array}{l}70.8^{\dagger} \\
75.0 \\
74.8 \\
71.7\end{array}$ \\
\hline
\end{tabular}

1 Money market instruments

Sources: National Statistics,

2 All loans secured on dwellings and all finance leasing are treated as long Bank of England

erm loans

3 Monetary financial institutions

4 Other than direct investment loans, loans secured on dwellings and loans used for finance leasing. 


\begin{tabular}{|c|c|c|c|c|c|c|c|c|c|c|c|c|c|c|c|c|c|}
\hline & \multicolumn{17}{|c|}{ Financial assets } \\
\hline & \multicolumn{6}{|c|}{ Currency and deposits } & \multicolumn{10}{|c|}{ Securities other than shares } & \multirow[b]{3}{*}{ Total } \\
\hline & \multirow[b]{2}{*}{$\begin{array}{r}\text { Curren- } \\
\text { cy }\end{array}$} & \multirow[b]{2}{*}{$\begin{array}{r}\text { Deposi- } \\
\text { ts with } \\
\text { UK } \\
\text { MFI's }^{1}\end{array}$} & \multirow[b]{2}{*}{$\begin{array}{r}\text { of } \\
\text { which } \\
\text { FC } \\
\text { deposi- } \\
\text { ts }\end{array}$} & \multirow[b]{2}{*}{$\begin{array}{r}\text { Deposi- } \\
\text { ts with } \\
\text { Rest of } \\
\text { the } \\
\text { World } \\
\text { MFl's }^{1}\end{array}$} & \multirow[b]{2}{*}{$\begin{array}{r}\text { Deposi- } \\
\text { ts } \\
\text { other } \\
\text { than } \\
\text { with } \\
\text { MFI's }\end{array}$} & \multirow[b]{2}{*}{ Total } & \multicolumn{5}{|c|}{ Short term MMl's ${ }^{2}$ issued } & \multicolumn{4}{|c|}{ Bonds issued } & \multirow[b]{2}{*}{$\begin{array}{r}\text { Financ- } \\
\text { ial } \\
\text { deriva- } \\
\text { tives }\end{array}$} & \\
\hline & & & & & & & $\begin{array}{r}\text { by UK } \\
\text { general } \\
\text { govern- } \\
\text { ment }\end{array}$ & $\begin{array}{r}\text { UK } \\
\text { local } \\
\text { author- } \\
\text { ity } \\
\text { bills }\end{array}$ & $\begin{array}{c}\text { by UK } \\
\text { MFI's }\end{array}$ & $\begin{array}{r}\text { by } \\
\text { other } \\
\text { UK } \\
\text { reside- } \\
\text { nts }\end{array}$ & $\begin{array}{l}\text { by rest } \\
\text { of the } \\
\text { world }\end{array}$ & $\begin{array}{r}\text { by UK } \\
\text { central } \\
\text { govern- } \\
\text { ment }\end{array}$ & $\begin{array}{r}\text { by UK } \\
\text { local } \\
\text { author- } \\
\text { ities }\end{array}$ & $\begin{array}{r}\text { by } \\
\text { other } \\
\text { UK } \\
\text { reside- } \\
\text { nts }\end{array}$ & $\begin{array}{r}\text { by the } \\
\text { rest of } \\
\text { the } \\
\text { world }\end{array}$ & & \\
\hline & $\mathrm{F} 21$ & $\mathrm{~F} 221$ & $\mathrm{~F} 2212$ & F229 & F29 & $\mathrm{F} 2$ & F3311 & F3312 & F3315 & F3316 & F3319 & F3321 & F3322 & F3326 & F3329 & F34 & F3 \\
\hline $\begin{array}{l}2004 \\
2005 \\
2006 \\
2007 \\
2008\end{array}$ & $\begin{array}{r}\text { NNMQ } \\
33.7 \\
35.6 \\
37.4 \\
39.7 \\
42.7\end{array}$ & $\begin{array}{r}\text { NNMS } \\
713.0 \\
770.3 \\
836.0 \\
903.5 \\
951.0\end{array}$ & $\begin{array}{r}\text { NNMU } \\
3.1 \\
3.4 \\
3.9 \\
4.9 \\
6.3\end{array}$ & $\begin{array}{r}\text { NNMW } \\
50.8 \\
57.7 \\
63.3 \\
75.6 \\
83.3\end{array}$ & $\begin{array}{r}\text { NNMX } \\
68.7 \\
72.9 \\
79.0 \\
84.8 \\
96.8\end{array}$ & $\begin{array}{r}\text { NNMP } \\
866.2 \\
936.5 \\
1015.8 \\
1103.6 \\
1173.8\end{array}$ & $\begin{array}{r}\text { NNNA } \\
- \\
- \\
- \\
- \\
-\end{array}$ & $\begin{array}{r}\text { NNNE } \\
- \\
- \\
- \\
- \\
-\end{array}$ & $\begin{array}{r}\text { NNNF } \\
2.5 \\
1.9 \\
3.1 \\
4.6 \\
6.2\end{array}$ & $\begin{array}{r}\text { NNNK } \\
0.5 \\
0.4 \\
0.5 \\
0.4 \\
-0.3\end{array}$ & $\begin{array}{r}\text { NNNL } \\
- \\
- \\
- \\
- \\
-\end{array}$ & $\begin{array}{r}\text { NNNN } \\
31.0 \\
37.5 \\
18.0 \\
12.2 \\
14.7\end{array}$ & $\begin{array}{r}\text { NNNQ } \\
0.3 \\
0.4 \\
0.7 \\
0.9 \\
0.9\end{array}$ & $\begin{array}{r}\text { NNNU } \\
4.9 \\
5.1 \\
5.3 \\
5.5 \\
5.7\end{array}$ & $\begin{array}{r}\text { NNNV } \\
7.7 \\
7.7 \\
7.5 \\
7.6 \\
8.5\end{array}$ & $\begin{array}{r}\text { NNNW } \\
- \\
-\overline{6} \\
0.3 \\
1.3 \\
2.5\end{array}$ & $\begin{array}{r}\text { NNMY } \\
47.0 \\
53.0 \\
35.8 \\
32.5 \\
38.2\end{array}$ \\
\hline $\begin{array}{r}2007 \text { Q2 } \\
\text { Q3 } \\
\text { Q4 }\end{array}$ & $\begin{array}{l}37.0 \\
37.8 \\
39.7\end{array}$ & $\begin{array}{l}872.7 \\
887.1 \\
903.5\end{array}$ & $\begin{array}{l}4.1 \\
4.3 \\
4.9\end{array}$ & $\begin{array}{l}71.2 \\
72.3 \\
75.6\end{array}$ & $\begin{array}{l}82.2 \\
83.3 \\
84.8\end{array}$ & $\begin{array}{l}1063.0 \\
1080.4 \\
1103.6\end{array}$ & $\begin{array}{l}- \\
- \\
-\end{array}$ & $\begin{array}{l}- \\
- \\
-\end{array}$ & $\begin{array}{l}3.3 \\
5.0 \\
4.6\end{array}$ & $\begin{array}{l}0.3 \\
0.3 \\
0.4\end{array}$ & $\begin{array}{l}- \\
- \\
-\end{array}$ & $\begin{array}{l}13.8 \\
13.1 \\
12.2\end{array}$ & $\begin{array}{l}0.8 \\
0.8 \\
0.9\end{array}$ & $\begin{array}{l}5.4 \\
5.5 \\
5.5\end{array}$ & $\begin{array}{l}7.5 \\
7.5 \\
7.6\end{array}$ & $\begin{array}{l}0.9 \\
0.7 \\
1.3\end{array}$ & $\begin{array}{l}32.0 \\
32.9 \\
32.5\end{array}$ \\
\hline $\begin{array}{r}2008 \text { Q1 } \\
\text { Q2 } \\
\text { Q3 } \\
\text { Q4 }\end{array}$ & $\begin{array}{l}39.0 \\
39.6 \\
39.4 \\
42.7\end{array}$ & $\begin{array}{l}920.7 \\
939.4 \\
941.6 \\
951.0\end{array}$ & $\begin{array}{l}5.2 \\
5.3 \\
5.1 \\
6.3\end{array}$ & $\begin{array}{l}82.7 \\
79.8 \\
79.3 \\
83.3\end{array}$ & $\begin{array}{l}85.9 \\
86.9 \\
90.6 \\
96.8\end{array}$ & $\begin{array}{ll}1 & 128.3 \\
1 & 145.7 \\
1 & 150.8 \\
1 & 173.8\end{array}$ & $\begin{array}{l}- \\
- \\
- \\
-\end{array}$ & $\begin{array}{l}- \\
- \\
- \\
-\end{array}$ & $\begin{array}{l}5.8 \\
6.0 \\
4.9 \\
6.2\end{array}$ & $\begin{array}{r}0.4 \\
- \\
-0.3 \\
-0.3\end{array}$ & $\begin{array}{l}- \\
- \\
- \\
-\end{array}$ & $\begin{array}{r}7.3 \\
10.8 \\
17.7 \\
14.7\end{array}$ & $\begin{array}{l}0.8 \\
0.8 \\
0.8 \\
0.9\end{array}$ & $\begin{array}{l}5.6 \\
5.6 \\
5.7 \\
5.7\end{array}$ & $\begin{array}{l}7.7 \\
7.7 \\
7.8 \\
8.5\end{array}$ & $\begin{array}{l}1.1 \\
1.2 \\
1.6 \\
2.5\end{array}$ & $\begin{array}{l}28.8 \\
32.2 \\
38.1 \\
38.2\end{array}$ \\
\hline $\begin{array}{r}2009 \text { Q1 } \\
\text { Q2 } \\
\text { Q3 } \\
\text { Q4 }\end{array}$ & $\begin{array}{l}43.0 \\
42.9 \\
43.8 \\
44.1\end{array}$ & $\begin{array}{l}952.5 \\
960.7 \\
963.9 \\
971.5\end{array}$ & $\begin{array}{l}5.4 \\
4.7 \\
5.2 \\
5.7\end{array}$ & $\begin{array}{l}75.0 \\
71.8 \\
73.4 \\
66.4\end{array}$ & $\begin{array}{l}99.0 \\
97.6 \\
96.3 \\
98.9\end{array}$ & $\begin{array}{ll}1 & 169.5 \\
1 & 173.0 \\
1 & 177.3 \\
1 & 180.9\end{array}$ & $\begin{array}{l}- \\
- \\
- \\
-\end{array}$ & $\begin{array}{l}- \\
- \\
- \\
-\end{array}$ & $\begin{array}{l}7.0 \\
7.2 \\
6.9 \\
5.6\end{array}$ & $\begin{array}{r}0.1 \\
- \\
- \\
-\end{array}$ & $\begin{array}{l}- \\
- \\
- \\
-\end{array}$ & $\begin{array}{r}13.1 \\
11.2 \\
9.4 \\
10.0\end{array}$ & $\begin{array}{l}0.6 \\
0.5 \\
0.4 \\
0.5\end{array}$ & $\begin{array}{l}5.8 \\
5.8 \\
5.9 \\
5.9\end{array}$ & $\begin{array}{l}8.3 \\
8.1 \\
8.2 \\
8.2\end{array}$ & $\begin{array}{l}2.2 \\
1.5 \\
1.8 \\
2.6\end{array}$ & $\begin{array}{l}37.2 \\
34.3 \\
32.6 \\
32.9\end{array}$ \\
\hline $\begin{array}{r}2010 \text { Q1 } \\
\text { Q2 } \\
\text { Q3 } \\
\text { Q4 }\end{array}$ & $\begin{array}{l}44.6 \\
46.5 \\
46.1 \\
48.3\end{array}$ & $\begin{array}{l}982.8 \\
988.3 \\
987.0 \\
998.7\end{array}$ & $\begin{array}{l}6.1 \\
5.6 \\
5.7 \\
5.6\end{array}$ & $\begin{array}{l}69.4^{\dagger} \\
69.2 \\
69.9 \\
69.1\end{array}$ & $\begin{array}{l}100.6 \\
103.1 \\
103.5 \\
101.4\end{array}$ & $\begin{array}{ll}1 & 197.5^{\dagger} \\
1 & 207.1 \\
1 & 206.6 \\
1 & 217.5\end{array}$ & $\begin{array}{l}- \\
- \\
- \\
-\end{array}$ & $\begin{array}{l}- \\
- \\
- \\
-\end{array}$ & $\begin{array}{l}5.1^{\dagger} \\
4.8 \\
5.8 \\
4.0\end{array}$ & $\begin{array}{l}0.1 \\
0.1 \\
0.1 \\
0.1\end{array}$ & $\begin{array}{l}- \\
- \\
- \\
-\end{array}$ & $\begin{array}{l}11.9^{\dagger} \\
10.9 \\
10.1 \\
12.4\end{array}$ & $\begin{array}{l}0.5 \\
0.6 \\
0.5 \\
0.4\end{array}$ & $\begin{array}{l}6.0 \\
6.0 \\
6.1 \\
6.1\end{array}$ & $\begin{array}{l}8.3 \\
8.3 \\
8.3 \\
8.3\end{array}$ & $\begin{array}{l}3.2 \\
2.7 \\
3.6 \\
2.7\end{array}$ & $\begin{array}{l}35.1^{\dagger} \\
33.4 \\
34.4 \\
34.1\end{array}$ \\
\hline
\end{tabular}

Financial assets - continued

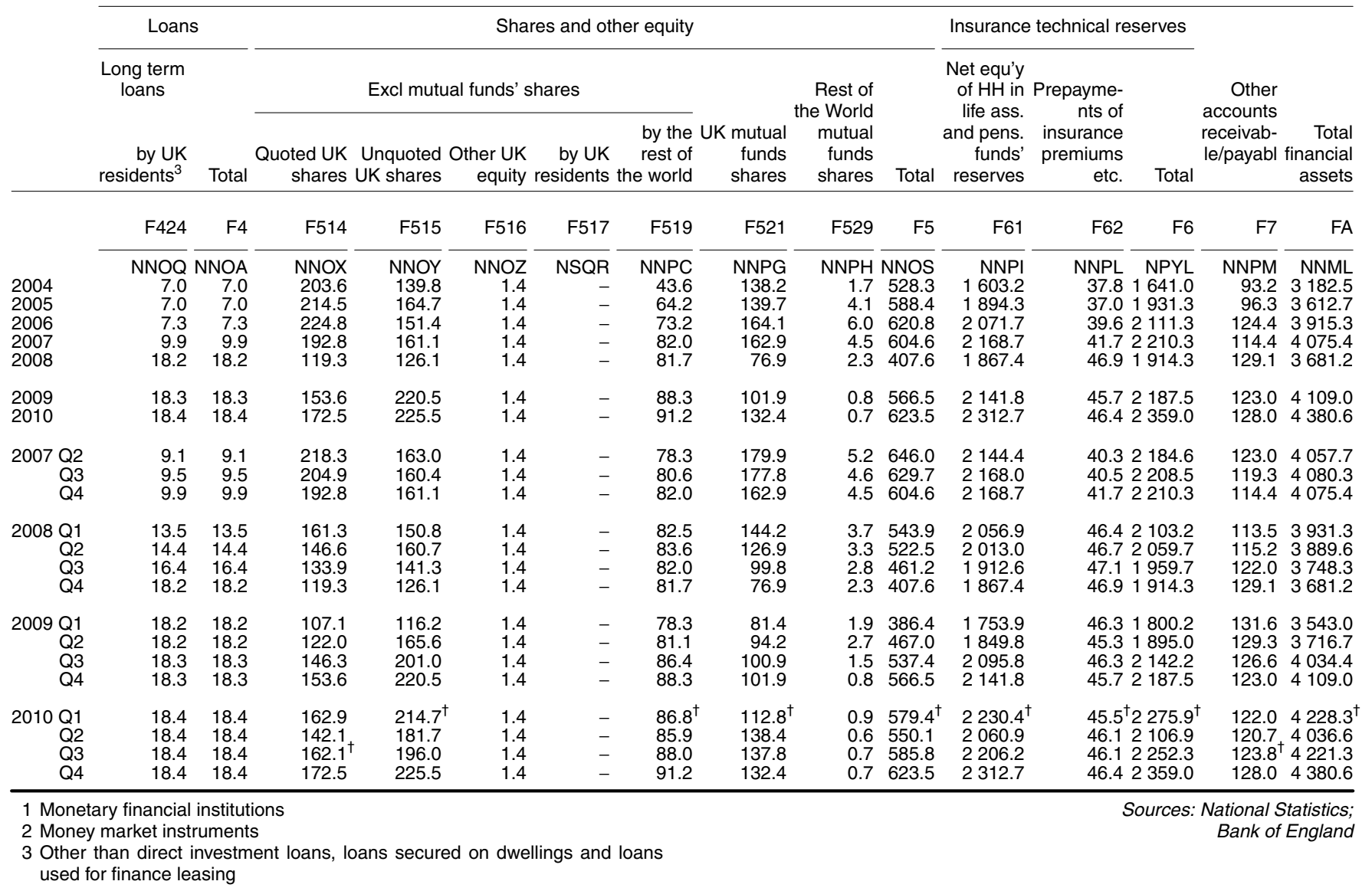




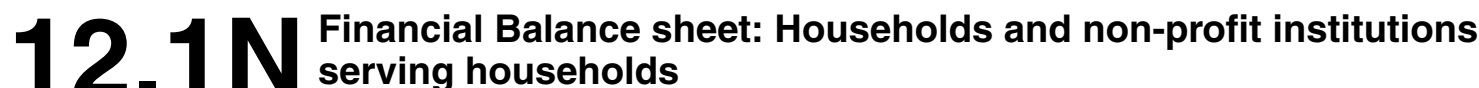

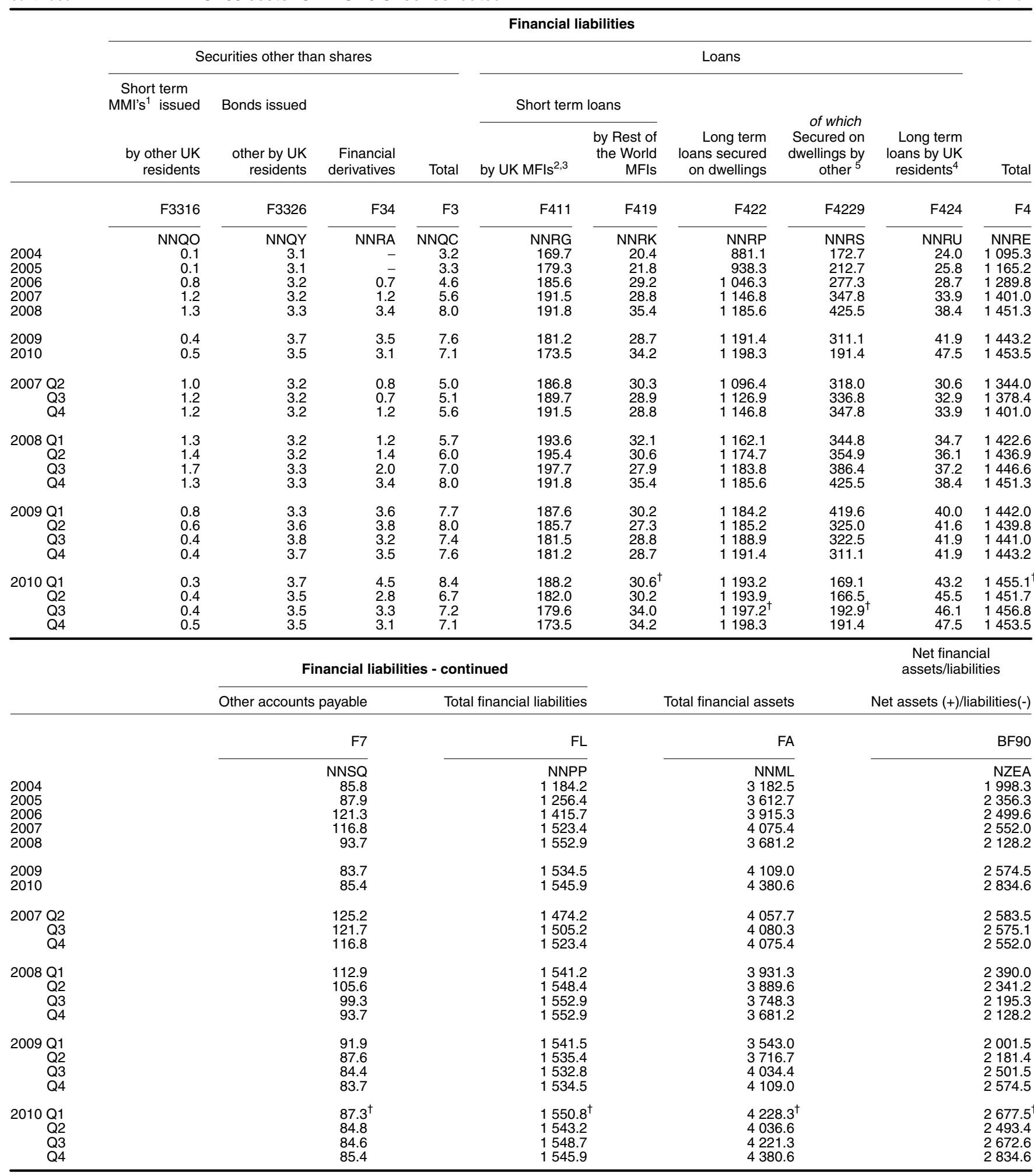

1 Money market instruments

2 All loans secured on dwellings and all finance leasing are treated as long

Sources: National Statistics, term loans

3 Monetary financial institutions

4 Other than direct investment loans, loans secured on dwellings and laons

for finance leasing

5 Reflects Housing Association reclassification in line with revisions policy

back to 2005 Q1. 


\begin{tabular}{|c|c|c|c|c|c|c|c|c|c|c|c|c|c|}
\hline & \multicolumn{13}{|c|}{ Financial assets } \\
\hline & \multicolumn{5}{|c|}{ Currency and deposits } & \multicolumn{8}{|c|}{ Securities other than shares } \\
\hline & \multirow[b]{2}{*}{ Currency } & \multirow[b]{2}{*}{$\begin{array}{c}\text { Deposits } \\
\text { with UK } \\
\text { MFI's }^{1}\end{array}$} & \multirow[b]{2}{*}{$\begin{array}{r}\text { of which } \\
\text { FC } \\
\text { deposits }\end{array}$} & \multirow[b]{2}{*}{$\begin{array}{r}\text { Deposits } \\
\text { other than } \\
\text { with MFl's }\end{array}$} & \multirow[b]{2}{*}{ Total } & \multicolumn{3}{|c|}{ Short term MMl's ${ }^{2}$ issued } & \multicolumn{4}{|c|}{ Bonds issued } & \multirow[b]{2}{*}{ Total } \\
\hline & & & & & & $\begin{array}{r}\text { by UK } \\
\text { general } \\
\text { government }\end{array}$ & $\begin{array}{l}\text { by UK } \\
\text { MFI's }\end{array}$ & $\begin{array}{r}\text { by other } \\
\text { UK } \\
\text { residents }\end{array}$ & $\begin{array}{r}\text { by UK } \\
\text { central } \\
\text { government }\end{array}$ & $\begin{array}{r}\text { by UK } \\
\text { local } \\
\text { authoriti- } \\
\text { es }\end{array}$ & $\begin{array}{l}\text { Medium } \\
\text { term bonds } \\
\text { issued by } \\
\text { UK MFI's' }^{1}\end{array}$ & $\begin{array}{r}\text { Other by } \\
\text { UK } \\
\text { residents }\end{array}$ & \\
\hline & $\mathrm{F} 21$ & F221 & F2212 & F29 & $\mathrm{F} 2$ & F3311 & F3315 & F3316 & F3321 & F3322 & F3325 & F3326 & F3 \\
\hline $\begin{array}{l}2004 \\
2005 \\
2006 \\
2007 \\
2008\end{array}$ & $\begin{array}{r}\text { NLCW } \\
1.4 \\
1.4 \\
1.5 \\
1.5 \\
1.5\end{array}$ & $\begin{array}{r}\text { NLCY } \\
1855.2 \\
2199.8 \\
2370.7 \\
3143.4 \\
3597.2\end{array}$ & $\begin{array}{r}\text { NLDA } \\
1570.0 \\
1561.6 \\
1974.2 \\
2531.0 \\
3031.5\end{array}$ & $\begin{array}{r}\text { NLDD } \\
1.0 \\
0.9 \\
1.4 \\
1.1 \\
0.8\end{array}$ & $\begin{array}{r}\text { NLCV } \\
1857.6 \\
2202.1 \\
2373.6 \\
3146.1 \\
3599.5\end{array}$ & $\begin{array}{r}\text { NLDG } \\
3.8 \\
2.8 \\
3.5 \\
7.2 \\
21.0\end{array}$ & $\begin{array}{l}\text { NLDL } \\
130.7 \\
136.1 \\
162.0 \\
183.7 \\
202.3\end{array}$ & $\begin{array}{r}\text { NLDQ } \\
22.5 \\
22.2 \\
16.3 \\
19.1 \\
29.9\end{array}$ & $\begin{array}{r}\text { NLDT } \\
83.8 \\
110.7 \\
135.5 \\
160.2 \\
200.0\end{array}$ & $\begin{array}{r}\text { NLDW } \\
- \\
- \\
- \\
- \\
-\end{array}$ & $\begin{array}{r}\text { NLDX } \\
70.9 \\
95.5 \\
114.9 \\
167.0 \\
200.2\end{array}$ & $\begin{array}{l}\text { NLEA } \\
361.8 \\
463.4 \\
529.1 \\
605.7 \\
767.0\end{array}$ & $\begin{array}{r}\text { NLDE } \\
673.6 \\
830.7 \\
1851.6 \\
2535.1 \\
5335.7\end{array}$ \\
\hline $\begin{array}{l}2009 \\
2010\end{array}$ & $\begin{array}{l}1.4 \\
1.4\end{array}$ & $\begin{array}{l}2976.3 \\
3067.0\end{array}$ & $\begin{array}{l}2475.1 \\
2584.9\end{array}$ & $\begin{array}{l}1.0 \\
1.3\end{array}$ & $\begin{array}{l}2978.7 \\
3069.7\end{array}$ & $\begin{array}{l}19.4 \\
27.6\end{array}$ & $\begin{array}{l}267.8 \\
205.5\end{array}$ & $\begin{array}{l}21.5 \\
25.4\end{array}$ & $\begin{array}{l}224.3 \\
309.2\end{array}$ & - & $\begin{array}{l}208.0 \\
204.0\end{array}$ & $\begin{array}{l}854.5 \\
847.4\end{array}$ & $\begin{array}{l}3717.3 \\
3833.6\end{array}$ \\
\hline $\begin{array}{r}2007 \text { Q2 } \\
\text { Q3 } \\
\text { Q4 }\end{array}$ & $\begin{array}{l}1.8 \\
2.0 \\
1.5\end{array}$ & $\begin{array}{l}2713.8 \\
2899.9 \\
3143.4\end{array}$ & $\begin{array}{l}2221.8 \\
2361.0 \\
2531.0\end{array}$ & $\begin{array}{l}0.6 \\
0.7 \\
1.1\end{array}$ & $\begin{array}{l}2716.2 \\
2902.6 \\
3146.1\end{array}$ & $\begin{array}{l}4.9 \\
5.8 \\
7.2\end{array}$ & $\begin{array}{l}171.0 \\
187.1 \\
183.7\end{array}$ & $\begin{array}{l}12.7 \\
17.1 \\
19.1\end{array}$ & $\begin{array}{l}145.9 \\
149.9 \\
160.2\end{array}$ & $\begin{array}{l}- \\
- \\
-\end{array}$ & $\begin{array}{l}147.8 \\
162.2 \\
167.0\end{array}$ & $\begin{array}{l}545.0 \\
557.3 \\
605.7\end{array}$ & $\begin{array}{l}1980.2 \\
2220.5 \\
2535.1\end{array}$ \\
\hline $\begin{array}{r}2008 \text { Q1 } \\
\text { Q2 } \\
\text { Q3 } \\
\text { Q4 }\end{array}$ & $\begin{array}{l}1.6 \\
1.8 \\
2.0 \\
1.5\end{array}$ & $\begin{array}{ll}3 & 412.3 \\
3 & 045.8 \\
3 & 331.7 \\
3 & 597.2\end{array}$ & $\begin{array}{l}2791.9 \\
2459.8 \\
2723.3 \\
3031.5\end{array}$ & $\begin{array}{l}0.3 \\
0.1 \\
0.4 \\
0.8\end{array}$ & $\begin{array}{ll}3 & 414.1 \\
3 & 047.7 \\
3 & 334.2 \\
3 & 599.5\end{array}$ & $\begin{array}{r}7.9 \\
10.7 \\
16.6 \\
21.0\end{array}$ & $\begin{array}{l}229.4 \\
219.2 \\
217.7 \\
202.3\end{array}$ & $\begin{array}{l}19.1 \\
19.4 \\
22.1 \\
29.9\end{array}$ & $\begin{array}{l}162.1 \\
168.0 \\
191.1 \\
200.0\end{array}$ & $\begin{array}{l}- \\
- \\
- \\
-\end{array}$ & $\begin{array}{l}167.7 \\
171.3 \\
168.6 \\
200.2\end{array}$ & $\begin{array}{l}628.7 \\
690.5 \\
713.7 \\
767.0\end{array}$ & $\begin{array}{ll}3 & 229.8 \\
3 & 272.9 \\
3 & 438.9 \\
5 & 335.7\end{array}$ \\
\hline $\begin{array}{r}2009 \text { Q1 } \\
\text { Q2 } \\
\text { Q3 } \\
\text { Q4 }\end{array}$ & $\begin{array}{l}1.5 \\
1.7 \\
1.8 \\
1.4\end{array}$ & $\begin{array}{ll}3 & 434.6 \\
3 & 028.7 \\
3 & 054.9 \\
2 & 976.3\end{array}$ & $\begin{array}{l}2857.7 \\
2479.4 \\
2535.0 \\
2475.1\end{array}$ & $\begin{array}{l}0.8 \\
0.5 \\
0.6 \\
1.0\end{array}$ & $\begin{array}{ll}3 & 436.8 \\
3 & 030.9 \\
3 & 057.3 \\
2 & 978.7\end{array}$ & $\begin{array}{l}25.1 \\
30.3 \\
21.4 \\
19.4\end{array}$ & $\begin{array}{l}259.7 \\
253.4 \\
277.6 \\
267.8\end{array}$ & $\begin{array}{l}28.2 \\
23.3 \\
24.3 \\
21.5\end{array}$ & $\begin{array}{l}215.9 \\
207.5 \\
216.9 \\
224.3\end{array}$ & $\begin{array}{l}- \\
- \\
- \\
-\end{array}$ & $\begin{array}{l}189.3 \\
194.7 \\
212.3 \\
208.0\end{array}$ & $\begin{array}{l}746.9 \\
782.5 \\
845.4 \\
854.5\end{array}$ & $\begin{array}{l}5235.1 \\
3 \quad 953.7 \\
4098.4 \\
3717.3\end{array}$ \\
\hline $\begin{array}{r}2010 \text { Q1 } \\
\text { Q2 } \\
\text { Q3 } \\
\text { Q4 }\end{array}$ & $\begin{array}{l}1.4 \\
1.7 \\
1.8 \\
1.4\end{array}$ & $\begin{array}{ll}3 & 136.7 \\
3 & 067.9^{\dagger} \\
3 & 085.8 \\
3 & 067.0\end{array}$ & $\begin{array}{l}2624.5 \\
2557.1^{\dagger} \\
2579.3 \\
2584.9\end{array}$ & $\begin{array}{l}0.3 \\
0.2 \\
1.3 \\
1.3\end{array}$ & $\begin{array}{ll}3 & 138.3^{\dagger} \\
3 & 069.8^{\dagger} \\
3 & 089.0 \\
3 & 069.7\end{array}$ & $\begin{array}{l}29.2 \\
30.5 \\
32.7 \\
27.6\end{array}$ & $\begin{array}{l}267.1 \\
210.9^{\dagger} \\
233.9 \\
205.5\end{array}$ & $\begin{array}{l}26.5 \\
28.0 \\
27.0 \\
25.4\end{array}$ & $\begin{array}{l}243.6 \\
274.5 \\
302.6 \\
309.2\end{array}$ & $\begin{array}{l}- \\
- \\
- \\
-\end{array}$ & $\begin{array}{l}217.7 \\
206.8 \\
216.8 \\
204.0\end{array}$ & $\begin{array}{l}863.5^{\dagger} \\
865.5 \\
834.4 \\
847.4\end{array}$ & $\begin{array}{l}4000.5 \\
4276.8 \\
4471.0 \\
3833.6\end{array}$ \\
\hline
\end{tabular}

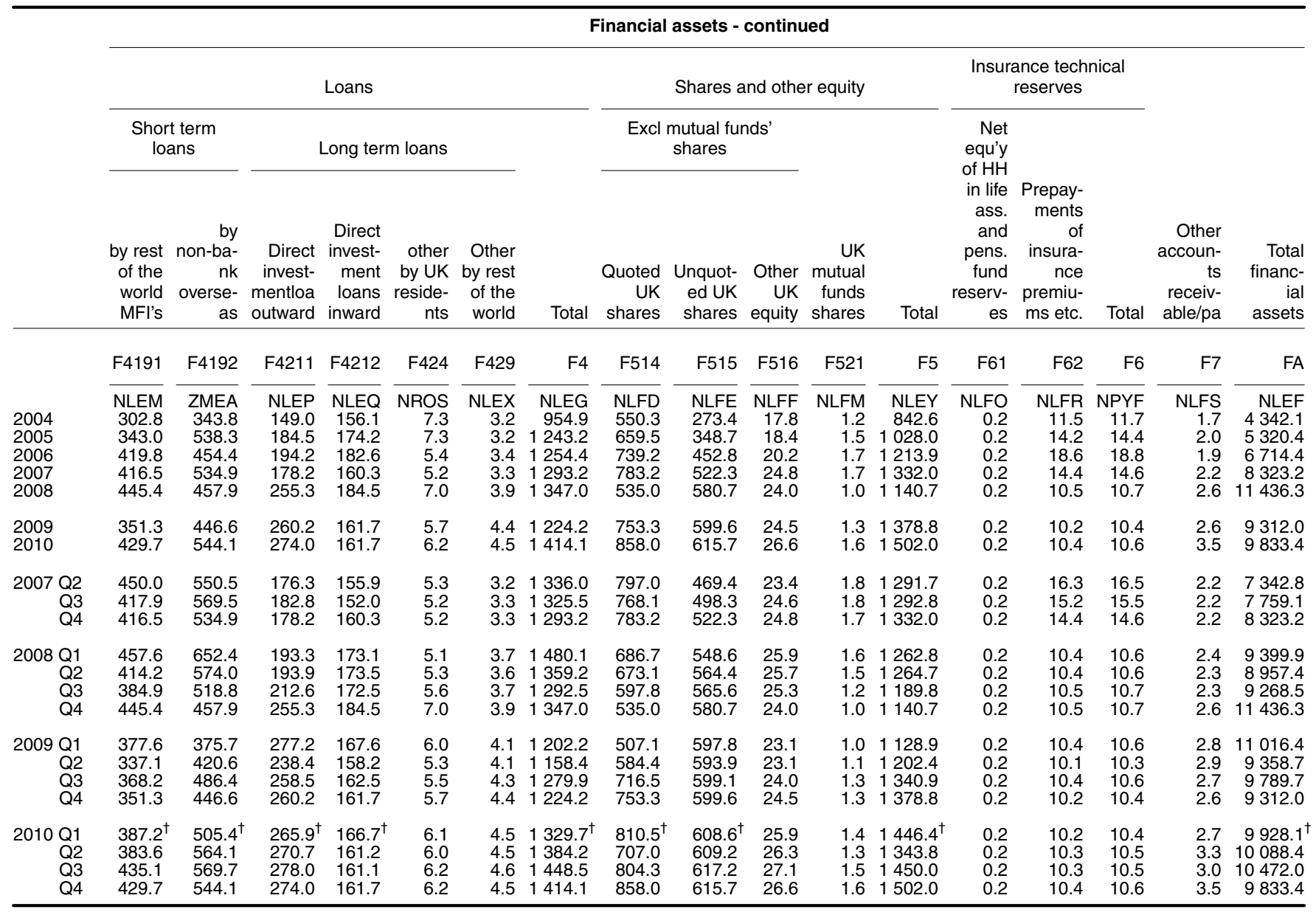




\begin{tabular}{|c|c|c|c|c|c|c|c|c|c|c|c|c|c|}
\hline & & & & & & & inancial lial & ities & & & & & \\
\hline & Curren & cy and dep & osits & Securities & other than & shares & & & Loa & & & & \\
\hline & & & & $\begin{array}{l}\text { Short term } \\
\text { MMl's }^{2} \\
\text { issued }\end{array}$ & $\begin{array}{l}\text { Long term } \\
\text { bonds } \\
\text { issued }\end{array}$ & & Short terr & n loans & & Long term & loans & & \\
\hline & Currency & $\begin{array}{l}\text { with rest } \\
\text { of the } \\
\text { world } \\
\text { MFI's }^{1}\end{array}$ & Total & $\begin{array}{r}\text { by the } \\
\text { Rest of } \\
\text { the World }\end{array}$ & $\begin{array}{r}\text { by the } \\
\text { Rest of } \\
\text { the World }\end{array}$ & Total & $\begin{array}{l}\text { Loans By } \\
\text { UK MFIs }^{3}\end{array}$ & $\begin{array}{r}\text { of which } \\
\text { Foreign } \\
\text { currency } \\
\text { loans }\end{array}$ & $\begin{array}{r}\text { Direct } \\
\text { investment } \\
\text { loans } \\
\text { outward }\end{array}$ & $\begin{array}{r}\text { Direct } \\
\text { investment } \\
\text { loans } \\
\text { inward }\end{array}$ & $\begin{array}{r}\text { Finance } \\
\text { leasing }\end{array}$ & $\begin{array}{l}\text { by UK } \\
\text { residents }{ }^{4}\end{array}$ & Total \\
\hline & $\mathrm{F} 21$ & F229 & $\mathrm{F} 2$ & F3319 & F3329 & F3 & $\mathrm{F} 411$ & F4112 & $\mathrm{F} 4211$ & F4212 & $\mathrm{F} 423$ & F424 & $\mathrm{F} 4$ \\
\hline $\begin{array}{l}2004 \\
2005 \\
2006 \\
2007 \\
2008\end{array}$ & $\begin{array}{r}\text { NLGA } \\
0.6 \\
0.6 \\
0.7 \\
0.8 \\
0.9\end{array}$ & $\begin{array}{r}\text { NLGG } \\
1605.3 \\
2055.5 \\
2189.5 \\
2768.0 \\
3042.5\end{array}$ & $\begin{array}{rr} & \text { NLFZ } \\
1 & 605.9 \\
2 & 056.1 \\
2 & 190.2 \\
2 & 768.8 \\
3 & 043.5\end{array}$ & $\begin{array}{r}\text { NLGV } \\
58.3 \\
63.8 \\
75.5 \\
76.4 \\
72.2\end{array}$ & $\begin{array}{r}\text { NLHF } \\
611.3 \\
717.2 \\
798.3 \\
903.5 \\
1075.4\end{array}$ & $\begin{array}{rr} & \text { NLGI } \\
669.7 \\
781.3 \\
1727.5 \\
2358.0 \\
5187.8\end{array}$ & $\begin{array}{r}\text { NLHM } \\
496.3 \\
642.0 \\
709.1 \\
955.9 \\
1127.8\end{array}$ & $\begin{array}{r}\text { NLHO } \\
448.9 \\
575.1 \\
621.3 \\
842.8 \\
1010.0\end{array}$ & $\begin{array}{l}\text { NLHT } \\
144.5 \\
145.6 \\
147.2 \\
194.5 \\
267.0\end{array}$ & $\begin{array}{r}\text { NLHU } \\
61.0 \\
77.0 \\
76.9 \\
75.5 \\
98.9\end{array}$ & $\begin{array}{r}\mathrm{NLHZ} \\
- \\
- \\
- \\
- \\
-\end{array}$ & $\begin{array}{r}\text { NROS } \\
7.3 \\
7.3 \\
5.4 \\
5.2 \\
7.0\end{array}$ & $\begin{array}{r}\text { NLHK } \\
709.2 \\
872.0 \\
938.6 \\
1231.0 \\
1500.7\end{array}$ \\
\hline $\begin{array}{r}2007 \text { Q2 } \\
\text { Q3 } \\
\text { Q4 }\end{array}$ & $\begin{array}{l}1.0 \\
0.7 \\
0.8\end{array}$ & $\begin{array}{l}2485.6 \\
2594.9 \\
2768.0\end{array}$ & $\begin{array}{l}2486.6 \\
2595.6 \\
2768.8\end{array}$ & $\begin{array}{l}86.8 \\
78.4 \\
76.4\end{array}$ & $\begin{array}{l}857.6 \\
863.4 \\
903.5\end{array}$ & $\begin{array}{ll}1 & 879.1 \\
2 & 076.1 \\
2 & 358.0\end{array}$ & $\begin{array}{l}842.1 \\
914.0 \\
955.9\end{array}$ & $\begin{array}{l}741.0 \\
803.0 \\
842.8\end{array}$ & $\begin{array}{l}155.8 \\
168.2 \\
194.5\end{array}$ & $\begin{array}{l}70.7 \\
73.9 \\
75.5\end{array}$ & $\begin{array}{l}- \\
- \\
-\end{array}$ & $\begin{array}{l}5.3 \\
5.2 \\
5.2\end{array}$ & $\begin{array}{ll}1 & 073.9 \\
1 & 161.3 \\
1 & 231.0\end{array}$ \\
\hline $\begin{array}{r}2008 \text { Q1 } \\
\text { Q2 } \\
\text { Q3 } \\
\text { Q4 }\end{array}$ & $\begin{array}{l}0.9 \\
1.1 \\
0.7 \\
0.9\end{array}$ & $\begin{array}{l}3081.5 \\
2750.9 \\
2882.9 \\
3042.5\end{array}$ & $\begin{array}{l}3082.4 \\
2751.9 \\
2883.7 \\
3043.5\end{array}$ & $\begin{array}{l}79.7 \\
77.5 \\
82.3 \\
72.2\end{array}$ & $\begin{array}{r}919.7 \\
898.6 \\
941.6 \\
1075.4\end{array}$ & $\begin{array}{ll}3 & 070.4 \\
3 & 040.0 \\
3 & 200.0 \\
5 & 187.8\end{array}$ & $\begin{array}{r}1059.5 \\
966.2 \\
1043.6 \\
1127.8\end{array}$ & $\begin{array}{r}940.1 \\
851.3 \\
923.8 \\
1010.0\end{array}$ & $\begin{array}{l}188.7 \\
193.5 \\
208.8 \\
267.0\end{array}$ & $\begin{array}{l}80.4 \\
85.0 \\
90.2 \\
98.9\end{array}$ & $\begin{array}{l}- \\
- \\
- \\
-\end{array}$ & $\begin{array}{l}5.1 \\
5.3 \\
5.6 \\
7.0\end{array}$ & $\begin{array}{ll}1 & 333.7 \\
1 & 250.0 \\
1 & 348.3 \\
1 & 500.7\end{array}$ \\
\hline $\begin{array}{r}2009 \text { Q1 } \\
\text { Q2 } \\
\text { Q3 } \\
\text { Q4 }\end{array}$ & $\begin{array}{l}0.8 \\
1.0 \\
0.7 \\
0.8\end{array}$ & $\begin{array}{l}2846.5 \\
2600.1 \\
2667.5 \\
2602.6\end{array}$ & $\begin{array}{l}2847.3 \\
2601.0 \\
2668.2 \\
2603.4\end{array}$ & $\begin{array}{l}76.8 \\
80.8 \\
91.5 \\
84.5\end{array}$ & $\begin{array}{ll}1 & 132.7 \\
1 & 084.2 \\
1 & 194.2 \\
1 & 176.1\end{array}$ & 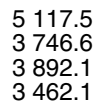 & $\begin{array}{r}1060.4 \\
917.7 \\
949.9 \\
903.5\end{array}$ & $\begin{array}{l}941.9 \\
812.0 \\
850.4 \\
806.9\end{array}$ & $\begin{array}{l}313.1 \\
267.7 \\
281.1 \\
292.0\end{array}$ & $\begin{array}{l}89.0 \\
78.8 \\
79.7 \\
77.7\end{array}$ & $\begin{array}{l}- \\
- \\
- \\
-\end{array}$ & $\begin{array}{l}6.0 \\
5.3 \\
5.5 \\
5.7\end{array}$ & $\begin{array}{ll}1 & 468.5 \\
1 & 269.5 \\
1 & 316.1 \\
1 & 278.9\end{array}$ \\
\hline $\begin{array}{r}2010 \text { Q1 } \\
\text { Q2 } \\
\text { Q3 } \\
\text { Q4 }\end{array}$ & $\begin{array}{l}0.8 \\
1.0^{\dagger} \\
1.2^{\dagger} \\
1.0\end{array}$ & $\begin{array}{l}2829.5^{\dagger} \\
2883.6 \\
2842.1 \\
2825.6\end{array}$ & $\begin{array}{l}2830.3^{\dagger} \\
2884.6 \\
2843.3 \\
2826.6\end{array}$ & $\begin{array}{l}78.0^{\dagger} \\
75.3 \\
96.1 \\
88.7\end{array}$ & $\begin{array}{ll}1 & 285.3^{\dagger} \\
1 & 253.6 \\
1 & 291.3 \\
1 & 300.3\end{array}$ & $\begin{array}{l}3778.4^{\dagger} \\
4041.6 \\
4260.3 \\
3664.8\end{array}$ & $\begin{array}{l}974.7 \\
927.5 \\
971.0^{\dagger} \\
971.8\end{array}$ & $\begin{array}{l}872.0 \\
828.1 \\
870.0^{\dagger} \\
867.8\end{array}$ & $\begin{array}{l}290.1^{\dagger} \\
287.4 \\
291.3 \\
276.9\end{array}$ & $\begin{array}{l}74.1^{\dagger} \\
76.2 \\
78.7 \\
79.4\end{array}$ & $\begin{array}{l}- \\
- \\
- \\
-\end{array}$ & $\begin{array}{l}6.1 \\
6.0 \\
6.2 \\
6.2\end{array}$ & $\begin{array}{ll}1 & 345.1 \\
1 & 297.0 \\
1 & 347.1 \\
1 & 334.4\end{array}$ \\
\hline
\end{tabular}

Financial liabilities - continued

Shares and other equity

Excl mutual funds' shares

\begin{tabular}{|c|c|c|c|c|c|c|c|}
\hline & $\begin{array}{r}\text { by the rest of the } \\
\text { world }\end{array}$ & Other UK equity & Total & $\begin{array}{l}\text { Other accounts } \\
\text { receivable/payable }\end{array}$ & $\begin{array}{r}\text { Total financial } \\
\text { liabilities }\end{array}$ & $\begin{array}{r}\text { Total financial } \\
\text { assets }\end{array}$ & $\begin{array}{r}\text { Net assets } \\
(+) / \text { /iabilities }(-)\end{array}$ \\
\hline & F519 & F52 & F5 & F7 & $\mathrm{FL}$ & FA & BF90 \\
\hline $\begin{array}{l}2004 \\
2005 \\
2006 \\
2007 \\
2008\end{array}$ & $\begin{array}{rr} & \text { NLIM } \\
1 & 128.8 \\
1 & 348.3 \\
1 & 459.0 \\
1 & 627.8 \\
1 & 587.4\end{array}$ & $\begin{array}{r}\text { NLIP } \\
1.7 \\
4.1 \\
6.0 \\
4.5 \\
2.3\end{array}$ & $\begin{array}{rr} & \text { NLIC } \\
1 & 130.5 \\
1 & 352.4 \\
1 & 465.0 \\
1 & 632.3 \\
1 & 589.7\end{array}$ & $\begin{array}{r}\text { NLIW } \\
3.6 \\
2.7 \\
3.8 \\
5.7 \\
6.8\end{array}$ & $\begin{array}{r}\text { NLHJ } \\
4119.0 \\
5064.6 \\
6325.2 \\
7995.7 \\
11328.5\end{array}$ & $\begin{array}{r}\text { NLEF } \\
4342.1 \\
5320.4 \\
6714.4 \\
8323.2 \\
11436.3\end{array}$ & $\begin{array}{l}\text { NLFK } \\
223.2 \\
255.7 \\
389.2 \\
327.5 \\
107.8\end{array}$ \\
\hline $\begin{array}{l}2009 \\
2010\end{array}$ & $\begin{array}{l}1648.7 \\
1787.8\end{array}$ & $\begin{array}{l}0.8 \\
0.7\end{array}$ & $\begin{array}{l}1649.5 \\
1788.5\end{array}$ & $\begin{array}{l}8.0 \\
8.5\end{array}$ & $\begin{array}{l}9001.9 \\
9622.7\end{array}$ & $\begin{array}{l}9312.0 \\
9833.4\end{array}$ & $\begin{array}{l}310.1 \\
210.7\end{array}$ \\
\hline $\begin{array}{r}2007 \text { Q2 } \\
\text { Q3 } \\
\text { Q4 }\end{array}$ & $\begin{array}{l}1560.9 \\
1588.8 \\
1627.8\end{array}$ & $\begin{array}{l}5.2 \\
4.6 \\
4.5\end{array}$ & $\begin{array}{l}1566.1 \\
1593.4 \\
1632.3\end{array}$ & $\begin{array}{l}3.8 \\
4.1 \\
5.7\end{array}$ & $\begin{array}{ll}7 & 009.6 \\
7 & 430.5 \\
7 & 995.7\end{array}$ & $\begin{array}{l}7342.8 \\
7759.1 \\
8323.2\end{array}$ & $\begin{array}{l}333.2 \\
328.6 \\
327.5\end{array}$ \\
\hline $\begin{array}{r}2008 \text { Q1 } \\
\text { Q2 } \\
\text { Q3 } \\
\text { Q4 }\end{array}$ & $\begin{array}{l}1572.6 \\
1576.6 \\
1565.8 \\
1587.4\end{array}$ & $\begin{array}{l}3.7 \\
3.3 \\
2.8 \\
2.3\end{array}$ & $\begin{array}{l}1576.3 \\
1580.0 \\
1568.7 \\
1589.7\end{array}$ & $\begin{array}{l}5.1 \\
4.9 \\
5.6 \\
6.8\end{array}$ & $\begin{array}{r}9068.0 \\
8626.8 \\
9006.3 \\
11328.5\end{array}$ & $\begin{array}{r}9399.9 \\
8957.4 \\
9268.5 \\
11436.3\end{array}$ & $\begin{array}{l}331.9 \\
330.6 \\
262.2 \\
107.8\end{array}$ \\
\hline $\begin{array}{r}2009 \text { Q1 } \\
\text { Q2 } \\
\text { Q3 } \\
\text { Q4 }\end{array}$ & $\begin{array}{ll}1 & 503.5 \\
1 & 512.9 \\
1 & 622.3 \\
1 & 648.7\end{array}$ & $\begin{array}{l}1.9 \\
2.7 \\
1.5 \\
0.8\end{array}$ & $\begin{array}{l}1505.4 \\
1515.5 \\
1623.9 \\
1649.5\end{array}$ & $\begin{array}{l}5.9 \\
6.5 \\
7.2 \\
8.0\end{array}$ & $\begin{array}{r}10944.7 \\
9139.2 \\
9507.5 \\
9001.9\end{array}$ & $\begin{array}{r}11016.4 \\
9358.7 \\
9789.7 \\
9312.0\end{array}$ & $\begin{array}{r}71.7 \\
219.5 \\
282.2 \\
310.1\end{array}$ \\
\hline $\begin{array}{r}2010 \text { Q1 } \\
\text { Q2 } \\
\text { Q3 } \\
\text { Q4 }\end{array}$ & $\begin{array}{ll}1 & 715.2^{\dagger} \\
1 & 616.5 \\
1 & 698.8 \\
1 & 787.8\end{array}$ & $\begin{array}{l}0.9 \\
0.6 \\
0.7 \\
0.7\end{array}$ & $\begin{array}{ll}1 & 716.0^{\dagger} \\
1 & 617.2 \\
1 & 699.5 \\
1 & 788.5\end{array}$ & $\begin{array}{l}6.6^{\dagger} \\
6.9 \\
7.8 \\
8.5\end{array}$ & $\begin{array}{r}9676.5^{\dagger} \\
9847.2 \\
10258.1 \\
9622.7\end{array}$ & $\begin{array}{r}9928.1^{\dagger} \\
10088.4 \\
10472.0 \\
9833.4\end{array}$ & $\begin{array}{l}251.6^{\dagger} \\
241.2 \\
213.9 \\
210.7\end{array}$ \\
\hline
\end{tabular}

Sources: National Statistics;

2 Money market instruments Bank of England

3 All loans secured on dwellings and all finance leasing are treated as long

4 Other than direct investment loans, loans secured on dwellings and loans

for finance leasing 


\begin{tabular}{|c|c|c|c|c|c|c|c|c|c|c|c|c|c|}
\hline & \multicolumn{4}{|c|}{ Liabilities } & \multicolumn{9}{|c|}{ Assets } \\
\hline & Banks & $\begin{array}{r}\text { Insurance } \\
\text { Companies }\end{array}$ & $\begin{array}{r}\text { Remaining } \\
\text { Financial } \\
\text { Instituti- } \\
\text { ons }\end{array}$ & $\begin{array}{r}\text { Private } \\
\text { Non-Finan- } \\
\text { cial } \\
\text { Companies }\end{array}$ & $\begin{array}{r}\text { Central } \\
\text { Government }\end{array}$ & $\begin{array}{r}\text { Local } \\
\text { Authoriti- } \\
\text { es }\end{array}$ & $\begin{array}{r}\text { Public } \\
\text { Corporati- } \\
\text { ons }\end{array}$ & Banks & $\begin{array}{r}\text { Insurance } \\
\text { Companies } \\
\text { \& Pension } \\
\text { Funds }\end{array}$ & $\begin{array}{r}\text { Remaining } \\
\text { Financial } \\
\text { Instituti- } \\
\text { ons }\end{array}$ & $\begin{array}{r}\text { Private } \\
\text { Non-Finan- } \\
\text { cial } \\
\text { Companies }\end{array}$ & $\begin{array}{r}\text { Households } \\
\& \\
\text { Charities }\end{array}$ & $\begin{array}{r}\text { Overseas } \\
\text { Holdings }\end{array}$ \\
\hline & NHYB & NJDP & NJWN & NLBZ & NIHN & NJGT & NKFZ & NHUX & NJAL & NJTJ & NKXV & NIUD & NLFD \\
\hline 2002 & 19358 & 33366 & 215605 & 857760 & 11 & 984 & 162 & 3310 & 397673 & 131918 & 8690 & 164695 & 418685 \\
\hline 2003 & 20806 & 36984 & 274190 & 1002020 & 11 & 1189 & 204 & 9565 & 443345 & 172963 & 9416 & 196911 & 500426 \\
\hline 2004 & 13969 & 43801 & 303733 & 1080203 & 11 & 801 & 243 & 8586 & 467803 & 201573 & 8762 & 203646 & 550281 \\
\hline 2005 & 11250 & 56920 & 340995 & 1232344 & 305 & 831 & - & 16650 & 485952 & 249987 & 16896 & 214458 & 659458 \\
\hline 2006 & 13460 & 73071 & 399218 & 1315541 & 565 & 1049 & - & 22732 & 493553 & 289650 & 32946 & 224776 & 739219 \\
\hline 2007 & 5045 & 67263 & 353376 & 1360477 & 549 & 899 & - & 26152 & 470471 & 282626 & 35102 & 192800 & 783198 \\
\hline 2008 & 107 & 39552 & 177128 & 909951 & 12818 & 314 & - & 11799 & 295836 & 125002 & 34706 & 119251 & 534953 \\
\hline 2009 & 97 & 43889 & 342645 & 1200278 & 54106 & 479 & - & 22363 & 351921 & 215034 & 48432 & 153595 & 753341 \\
\hline 2010 & .. & 47935 & 368039 & 1361334 & 54106 & 957 & - & .. & 361422 & 266860 & 54051 & 172474 & 857960 \\
\hline 2002 Q1 & 28971 & 50556 & 277430 & 1161066 & 14 & 1126 & 198 & 12194 & 543039 & 162050 & 14961 & 227773 & 556668 \\
\hline Q3 & 18765 & 27360 & 198417 & 829255 & 11 & 1024 & 149 & 2281 & 381000 & 121736 & 8932 & 161017 & 397740 \\
\hline Q4 & 19358 & 33366 & 215605 & 857760 & 11 & 984 & 162 & 3310 & 397673 & 131918 & 8690 & 164695 & 418685 \\
\hline 2003 Q1 & 15817 & 24739 & 208072 & 790914 & 11 & 951 & 163 & 3199 & 357742 & 125305 & 7722 & 152221 & 392254 \\
\hline Q2 & 19513 & 30871 & 247670 & 888016 & 11 & 1052 & 184 & 3205 & 405149 & 144546 & 8643 & 177933 & 445374 \\
\hline Q3 & 19766 & 34418 & 255981 & 917492 & 11 & 1078 & 189 & 4479 & 413751 & 155962 & 8816 & 182368 & 461038 \\
\hline Q4 & 20806 & 36984 & 274190 & 1002020 & 11 & 1189 & 204 & 9565 & 443345 & 172963 & 9416 & 196911 & 500426 \\
\hline 2004 Q1 & 19946 & 37681 & 267952 & 999846 & 11 & 862 & 210 & 6437 & 438991 & 177438 & 8983 & 193545 & 498996 \\
\hline Q2 & 20242 & 38968 & 266597 & 1019511 & 11 & 864 & 216 & 4881 & 437637 & 187413 & 8717 & 197151 & 508524 \\
\hline Q3 & 20668 & 38596 & 278271 & 1029239 & 11 & 786 & 207 & 7785 & 444784 & 188601 & 8491 & 196877 & 519330 \\
\hline Q4 & 13969 & 43801 & 303733 & 1080203 & 11 & 801 & 243 & 8586 & 467803 & 201573 & 8762 & 203646 & 550281 \\
\hline 2005 Q1 & 13856 & 47734 & 294220 & 1113555 & 259 & 843 & - & 7412 & 472171 & 203570 & 6538 & 204091 & 576097 \\
\hline Q2 & 9684 & 48596 & 308313 & 1159100 & 274 & 850 & - & 9625 & 475748 & 224199 & 9989 & 207744 & 599637 \\
\hline Q3 & 9809 & 51426 & 318328 & 1189868 & 290 & 924 & - & 16843 & 474679 & 237051 & 13414 & 210805 & 618223 \\
\hline Q4 & 11250 & 56920 & 340995 & 1232344 & 305 & 831 & - & 16650 & 485952 & 249987 & 16896 & 214458 & 659458 \\
\hline 2006 Q1 & 13918 & 65287 & 368988 & 1287139 & 561 & 1035 & - & 16259 & 507053 & 264631 & 20427 & 221279 & 707933 \\
\hline Q2 & 12804 & 60856 & 355635 & 1249788 & 541 & 1059 & - & 17590 & 476144 & 266246 & 24630 & 211259 & 685600 \\
\hline 2007 Q1 & 13075 & 70820 & & 1368681 & 563 & 1041 & - & 27644 & 462 & 227 & & 519 & 034 \\
\hline Q2 & 11330 & 68473 & 392321 & 1380660 & 553 & 961 & - & 26692 & 487702 & 296111 & 31115 & 218253 & 797028 \\
\hline Q3 & 6400 & 69423 & 370824 & 1356526 & 531 & 934 & - & 27827 & 481706 & 291709 & 32777 & 204919 & 768103 \\
\hline Q4 & 5045 & 67263 & 353376 & 1360477 & 549 & 899 & - & 26152 & 470471 & 282626 & 35102 & 192800 & 783198 \\
\hline 2008 Q1 & 3513 & 60042 & 299674 & 1202125 & 538 & 784 & - & 19445 & 418020 & 250772 & 34467 & 161277 & 686744 \\
\hline Q2 & 1804 & 46145 & 258272 & 1171167 & 560 & 702 & - & 24855 & 393483 & 210167 & 35269 & 146559 & 673108 \\
\hline Q3 & 1258 & 46446 & 255931 & 993482 & 311 & 662 & - & 17409 & 343124 & 176770 & 34935 & 133943 & 597765 \\
\hline Q4 & 107 & 39552 & 177128 & 909951 & 12818 & 314 & - & 11799 & 295836 & 125002 & 34706 & 119251 & 534953 \\
\hline 2009 Q1 & 98 & 29918 & 160440 & 850130 & 22166 & 20 & - & 11905 & 256303 & 117447 & 30893 & 107092 & 507122 \\
\hline Q2 & 87 & 33976 & 245721 & 927669 & 28910 & 295 & - & 16229 & 284519 & 148474 & 35005 & 121971 & 584412 \\
\hline Q3 & 88 & 47650 & 335268 & 1107584 & 28910 & 437 & - & 19231 & 348036 & 198998 & 44562 & 146253 & 716525 \\
\hline Q4 & 97 & 43889 & 342645 & 1200278 & 54106 & 479 & - & 22363 & 351921 & 215034 & 48432 & 153595 & 753341 \\
\hline 2010 Q1 & .. & 42825 & 369674 & 1270704 & 54106 & 71 & - & .. & $365109^{\dagger}$ & $228814^{\dagger}$ & 51323 & 162862 & $810495^{\dagger}$ \\
\hline Q2 &.. & 38948 & 333641 & 1108052 & 54106 & 413 & - & .. & 314557 & 206685 & 44681 & 142111 & 707005 \\
\hline Q3 & $\begin{array}{l}. . \\
.\end{array}$ & 47650 & 371959 & 1256319 & 54106 & $705^{\dagger}$ & - & .. & 348851 & 247259 & $50889^{\dagger}$ & $162058^{\dagger}$ & 804304 \\
\hline Q4 & .. & 47935 & 368039 & 1361334 & 54106 & 957 & - & .. & 361422 & 266860 & 54051 & 172474 & 857960 \\
\hline
\end{tabular}

